International Journal of Bifurcation and Chaos, Vol. 12, No. 5 (2002) 931-963

(c) World Scientific Publishing Company

\title{
SOME ANALYTICAL CRITERIA FOR LOCAL ACTIVITY OF TWO-PORT CNN WITH THREE OR FOUR STATE VARIABLES: ANALYSIS AND APPLICATIONS
}

\author{
LEQUAN MIN* and NA YU ${ }^{\dagger}$ \\ Department of Mathematics and Mechanics, \\ University of Science and Technology Beijing, Beijing 100083, P. R. China \\ *lqmin@sohu.com \\ †bd-yuna@sohu.com
}

Received January 30, 2001; Revised October 1, 2001

\begin{abstract}
The local activity principle of the Cellular Nonlinear Network (CNN) introduced by Chua [1997] has provided a powerful tool for studying the emergence of complex patterns in a homogeneous lattice formed by coupled cells. This paper presents some analytical criteria for the local activity of two-port CNN cells with three or four state variables. As a first application, a coupled excitable cell model (ECM) CNN is introduced, which has cells defined by the Chay equations representing ionic events in excitable membranes in terms of a Hodgkin-Huxley type formalism. The bifurcation diagram of the ECM CNN supplies a possible explanation for the mechanism of arrhythmia (from normal to abnormal until stopping) of excitable cells: the cell parameter is changed from an active unstable domain to an edge of chaos. The member potentials along fibers are simulated numerically, where oscillatory patterns, chaotic patterns as well as convergent patterns are observed. As a second application, a smoothed Chua's circuit (SCC) CNN with two ports is presented, whose prototype has been introduced by Chua as a dual-layer two-dimensional reaction-diffusion CNN in order to obtain Turing patterns. The bifurcation diagrams of the SCC CNN are the same as those with one port, which have only active unstable domains and edges of chaos. Numerical simulations show that in the active unstable parameter domains, the evolutions of the patterns of the state variables of the SCC CNNs can exhibit divergence, periodicity and chaos, where, in the parameter domains located in the edge of chaos, periodic patterns and divergent patterns are observed. These results demonstrate once again the effectiveness of the local activity theory in choosing the parameters for the emergence of complex patterns of CNNs.
\end{abstract}

Keywords: CNN; local activity; excitable cell; smoothed Chua's circuit; chaos.

\section{Introduction}

The study of CNNs is a multi-disciplinary area, first introduced by Chua and Yang [1988a, 1998b] after Hopfield proposed his fully-connected neural networks [Hopfield, 1985]. Hopfield network grows exponentially with the size of the array, which cannot be built physically even in a modest array size. However, CNN can be built by circuitry, with no electrical interconnections beyond a prescribed sphere of influence. Consequently, CNNs have been widely studied on both theoretical foundations [Chua \& Yang, 1998a; Chua \& Roska, 1990; Chua \& Wu, 1992; Joy \& Tavasnoglu, 1993; Gili, 1994; Thiran et al., 1995; Chua, 1997; Takahashi \& Chua, 1997; Min et al., 1999; Lin 
\& Shi, 1999] and image processing [Chua, 1997; Special issue, 1992; Special issue, 1993; Werblin et al., 1994; Werblin et al., 1996; Orzo et al., 1996; Li \& Min, 2000].

It is now known that CNNs can also be used for the study of complex patterns and structures, emerging from homogeneous media operating far from thermodynamic equilibrium [Chua, 1997, 1999]. In particular, some analytical criteria for local activity of CNNs have been successfully applied to the study of the dynamics of the CNNs [Dogaru \& Chua, 1998a, 1998b, 1998c; Min et al. 2000a, 2000b; Min \& Yu, 2000] related to the FitzHugh-Nagumo equation, the Brusselator equation, the Gierer-Meinhart equation, the Oregonator equation, the Hodgkin-Huxley equation, and the enzyme reaction equation. Some local activity criteria for "difference-equation" CNN has recently been set up, in [Sbitnev, et al., 2001].

In this paper, some analytical criteria for the local activity of two-port CNN cells with three and four state variables are introduced in Secs. 1 and 2 , respectively. As the first application, the criteria are used to calculate the bifurcation diagram of the ECM CNN with three state variables and two ports whose prototype - the three-dimensional Chay model - can generate chaotic action potentials and intracellular calcium concentrations [Chay, 1985]. The bifurcation diagram of the ECM CNNs shows that there does not exist a locally passive domain. Numerical simulations of the dynamics of the ECM CNNs show that one-period, multi-period, chaotic, and convergent trajectories can be generated if the parameters of the corresponding ECM CNNs vary from locally active and unstable domain to the edge of chaos. If the oscillations of the ECM CNNs are understood as the frequency of the heartbeat, the following relations are observed with parameter $g_{K, V}^{*}$ (the maximum conductance of voltage-sensitive $\mathrm{K}^{+}$channel divided by membrane capacitance) decreasing:

Normal heart rate $\rightarrow$ Multi-period heart rate

$\rightarrow$ Chaotic heart rate $\rightarrow$ Multi-period heart rate $\rightarrow$ Stop beating.

As the second application of the criteria, we add a new state variable equation to the piecewise linear dual-layer two-dimensional reaction-diffusion CNN [Chua, 1995], and smooth the CNN to a differentiable one, in a way similar to the case of the SCC CNN with one port [Min et al., 2000b]. Computer simulations show that the bifurcation diagrams of the SCC CNNs are the same as those of the SCC CNNs with one port [Min et al., 2000b]. Numerical simulations show that the dynamic patterns of the SCC CNN, whose parameters are located in locally active domains, bound with complex patterns - one-period, chaotic and divergent trajectories, particularly when selected parameters are located nearby the horizontal or the oblique edges of chaos in the bifurcation diagrams.

This paper is organized as follows: Section 2 states the basic local activity theory set up by [Chua, 1997]. Some analytical criteria for the local activity in two-port CNN cells with three and four state variables are presented in Secs. 3.1 and 4.1, respectively. The bifurcation diagrams of the ECM CNNs and the SCC CNNs are given in Secs. 3.2 and 4.2 , respectively. Sections 3.3 and 4.3 demonstrate the numerical simulations for ECM CNNs and SCC CNNs. Concluding remarks on the ECM CNNs and the SCC CNNs are addressed in Secs. 3.4 and 4.4, respectively.

\section{A Main Theorem of Local Activity}

Generally speaking, in a reaction-diffusion CNN, every $C(j, k, l)$ has $n$ state variables but only $m$ $(\leq n)$ state variables coupled directly to their nearest neighbors via "reaction-diffusion." Consequently, each cell $C(j, k, l)$ has the state equations [Chua, 1997]:

$$
\begin{aligned}
\dot{V}_{1}(j, k, l) & =f_{1}\left(V_{1}, V_{2}, \ldots, V_{n}\right)+D_{1} \nabla^{2} V_{1} \\
\dot{V}_{2}(j, k, l) & =f_{2}\left(V_{1}, V_{2}, \ldots, V_{n}\right)+D_{2} \nabla^{2} V_{2} \\
& \vdots \\
\dot{V}_{m}(j, k, l) & =f_{m}\left(V_{1}, V_{2}, \ldots, V_{n}\right)+D_{m} \nabla^{2} V_{m} \\
\dot{V}_{m+1}(j, k, l) & =f_{m+1}\left(V_{1}, V_{2}, \ldots, V_{n}\right) \\
& \vdots \\
\dot{V}_{n}(j, k, l) & =f_{n}\left(V_{1}, V_{2}, \ldots, V_{n}\right) \\
j & =1,2, \ldots, N_{x} \\
k & =1,2, \ldots, N_{y} \\
l & =1,2, \ldots, N_{z} .
\end{aligned}
$$

In a component form, Eq. (1) becomes

$$
\left\{\begin{array}{l}
\dot{\mathbf{V}}_{\mathbf{a}}=\mathbf{f}_{\mathbf{a}}\left(\mathbf{V}_{\mathbf{a}}, \mathbf{V}_{\mathbf{b}}\right)+\mathbf{I}_{\mathbf{a}} \\
\dot{\mathbf{V}}_{\mathbf{b}}=\mathbf{f}_{\mathbf{b}}\left(\mathbf{V}_{\mathbf{a}}, \mathbf{V}_{\mathbf{b}}\right)
\end{array}\right.
$$


where

$$
\begin{aligned}
\mathbf{V}_{\mathbf{a}} & =\left[V_{1}, V_{2}, \ldots, V_{m}\right]^{T} \\
\mathbf{V}_{\mathbf{b}} & =\left[V_{m+1}, V_{m+2}, \ldots, V_{n}\right]^{T} \\
\mathbf{f}_{\mathbf{a}} & =\left[f_{1}(\cdot), f_{2}(\cdot), \ldots, f_{m}(\cdot)\right]^{T} \\
\mathbf{f}_{\mathbf{b}} & =\left[f_{m+1}(\cdot), f_{m+2}(\cdot), \ldots, f_{n}(\cdot)\right]^{T} \\
\mathbf{I}_{\mathbf{a}} & =\mathbf{D}_{\mathbf{a}} \nabla^{2} \mathbf{V}_{\mathbf{a}} \\
& =\left[D_{1} \nabla^{2} V_{1}, D_{2} \nabla^{2} V_{2}, \ldots, D_{m} \nabla^{2} V_{m}\right]^{T} \\
\mathbf{D}_{\mathbf{a}} & =\operatorname{diag}\left[D_{1}, D_{2}, \ldots, D_{m}\right] .
\end{aligned}
$$

The cell equilibrium point $Q_{i}=\left(\overline{\mathbf{V}}_{\mathbf{a}}, \overline{\mathbf{V}}_{\mathbf{b}}\right)\left(\in \mathbb{R}^{n}\right)$ of Eq. (2) for the restricted local activity domain [Dogaru \& Chua, 1998a] can be determined, numerically or analytically, via

$$
\left\{\begin{array}{l}
\mathbf{f}_{\mathbf{a}}\left(\mathbf{V}_{\mathbf{a}}, \mathbf{V}_{\mathbf{b}}\right)=0 \\
\mathbf{f}_{\mathbf{b}}\left(\mathbf{V}_{\mathbf{a}}, \mathbf{V}_{\mathbf{b}}\right)=0 .
\end{array}\right.
$$

The Jacobian at an equilibrium point $Q_{i}$, for the restricted local activity domain, has the following form:

$$
\mathbf{J}\left(Q_{i}\right) \triangleq\left[a_{l k}\left(Q_{i}\right)\right] \triangleq\left[\begin{array}{ll}
\mathbf{A}_{\mathbf{a a}}\left(Q_{i}\right) & \mathbf{A}_{\mathbf{a b}}\left(Q_{i}\right) \\
\mathbf{A}_{\mathbf{b a}}\left(Q_{i}\right) & \mathbf{A}_{\mathbf{b b}}\left(Q_{i}\right)
\end{array}\right],
$$

where

$$
\begin{aligned}
\mathbf{A}_{\mathbf{a a}}\left(Q_{i}\right)= & {\left[\begin{array}{ccc}
\frac{\partial \mathbf{f}_{1}}{\partial V_{1}} & \cdots & \frac{\partial \mathbf{f}_{1}}{\partial V_{m}} \\
\cdots & \cdots & \cdots \\
\frac{\partial \mathbf{f}_{m}}{\partial V_{1}} & \cdots & \frac{\partial \mathbf{f}_{m}}{\partial V_{m}}
\end{array}\right] } \\
\mathbf{A}_{\mathbf{a b}}\left(Q_{i}\right)= & {\left[\begin{array}{ccc}
\frac{\partial \mathbf{f}_{1}}{\partial V_{m+1}} & \cdots & \frac{\partial \mathbf{f}_{1}}{\partial V_{n}} \\
\cdots & \cdots & \cdots \\
\frac{\partial \mathbf{f}_{m}}{\partial V_{m+1}} & \cdots & \frac{\partial \mathbf{f}_{m}}{\partial V_{n}}
\end{array}\right] } \\
\mathbf{A}_{\mathbf{b a}}\left(Q_{i}\right)= & {\left[\begin{array}{ccc}
\frac{\partial \mathbf{f}_{m+1}}{\partial V_{1}} & \cdots & \frac{\partial \mathbf{f}_{m+1}}{\partial V_{m}} \\
\cdots & \cdots & \cdots \\
\frac{\partial \mathbf{f}_{n}}{\partial V_{1}} & \cdots & \frac{\partial \mathbf{f}_{n}}{\partial V_{m}}
\end{array}\right] }
\end{aligned}
$$

$$
\mathbf{A}_{\mathbf{b b}}\left(Q_{i}\right)=\left[\begin{array}{ccc}
\frac{\partial \mathbf{f}_{m+1}}{\partial V_{m+1}} & \cdots & \frac{\partial \mathbf{f}_{m+1}}{\partial V_{n}} \\
\cdots & \cdots & \cdots \\
\frac{\partial \mathbf{f}_{n}}{\partial V_{m+1}} & \cdots & \frac{\partial \mathbf{f}_{n}}{\partial V_{n}}
\end{array}\right] .
$$

$a_{l k}\left(Q_{i}\right)^{\prime} s$ are called cell parameters.

The local state equations at the cell equilibrium point $Q_{i}$ are defined via [Chua, 1997]

$$
\begin{aligned}
\dot{\mathbf{V}}_{\mathbf{a}} & =\mathbf{A}_{\mathbf{a a}} \mathbf{V}_{\mathbf{a}}+\mathbf{A}_{\mathbf{a b}} \mathbf{V}_{\mathbf{b}}+\mathbf{I}_{\mathbf{a}} \\
\dot{\mathbf{V}}_{\mathbf{b}} & =\mathbf{A}_{\mathbf{b a}} \mathbf{V}_{\mathbf{a}}+\mathbf{A}_{\mathbf{b b}} \mathbf{V}_{\mathbf{b}},
\end{aligned}
$$

and

$$
\mathbf{Y}_{Q}(s) \triangleq\left(s \mathbf{I}-\mathbf{A}_{\mathbf{a a}}\right)-\mathbf{A}_{\mathbf{a b}}\left(s \mathbf{I}-\mathbf{A}_{\mathbf{b b}}\right)^{-1} \mathbf{A}_{\mathbf{b a}}
$$

is called the admittance matrix at $Q_{i}$.

Main Theorem on the Local Activity of CNN [Chua, 1997]. A two-port Reaction Diffusion CNN cell is locally active at a cell equilibrium point $Q \triangleq$ $\left(\overline{\mathbf{V}}_{\mathbf{a}}, \overline{\mathbf{V}}_{\mathbf{b}}, \overline{\mathbf{I}}_{\mathbf{a}}\right)$ if, and only if, its cell admittance $\mathbf{Y}_{Q}(s)$ at $Q$ satisfies at least one of the following four conditions:

(1) $\mathbf{Y}_{Q}(s)$ has a pole in $\operatorname{Re}[s]>0$.

(2) $\mathbf{Y}_{Q}^{H}(i \omega)=\mathbf{Y}_{Q}^{\dagger}(i \omega)+\mathbf{Y}_{Q}(i \omega)$ is not a positive semi-definite matrix at some $\omega=\omega_{0}$, where $\omega_{0}$ is any real number, and $\mathbf{Y}_{Q}^{\dagger}(s)$ is constructed by taking first the transpose of $\mathbf{Y}_{Q}(s)$, and then followed by the complex conjugate operation.

(3) $\mathbf{Y}_{Q}(s)$ has a simple pole $s=i \omega_{\rho}$ on the imaginary axis, where its associated residue matrix

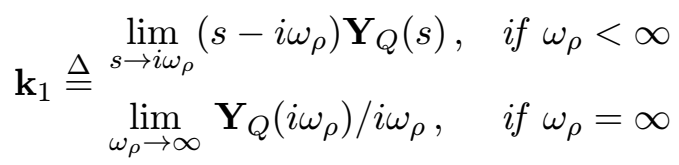

is either not a Hermitian matrix, or else not a positive semi-definite Hermitian matrix.

(4) $\mathbf{Y}_{Q}(s)$ has a multiple pole on the imaginary axis.

Definition 2.1. [Dogaru \& Chua, 1998; Min et al., 2000a]. (Edge of chaos with respect to the equilibrium point $Q_{i}$ ) A "Reaction-Diffusion" CNN with one "diffusion coefficient" $D_{1}$ (resp. two diffusion coefficients $D_{1}$ and $D_{2}$; or three diffusion coefficients $D_{1}, D_{2}$ and $D_{3}$ ) is said to be operating on the edge of chaos with respect to an equilibrium point $Q_{i}$ if, and only if, $Q_{i}$ is both locally active 
and stable when $\overline{\mathbf{I}}_{1}=0$ (resp. $\overline{\mathbf{I}}_{2}=0$ and $\overline{\mathbf{I}}_{3}=0$; or $\overline{\mathbf{I}}_{1}=0, \overline{\mathbf{I}}_{2}=0$ and $\overline{\mathbf{I}}_{3}=0$ ).

Remark 2.1. $Q_{i}$ is said to be stable if

(i) The real parts of the eigenvalues $\lambda$ of the Jacobian matrix $\mathbf{J}\left(Q_{i}\right)$ (4) are all less than zero, or

(ii) $\operatorname{Real}\left(\lambda_{i}\right)=0 \Rightarrow$ the multiplicity of $\lambda_{i}$ is equal to 1 .

By arbitrarily choosing parameters of CNNs within or nearby the edge of chaos domain, complex dynamic behaviors may become abundant, even with only a very small perturbations of parameters [Dogaru \& Chua, 1998a-1998c; Min et al., 2000a, 2000b; Min \& Yu, 2000].

\section{Local Activity Theory of the CNN with Three State Variables and Two Ports}

In the following subsections, we first introduce a set of analytical criteria for testing the local activity of the CNNs with three state variables and two-port (i.e. with two reaction-diffusion coefficients depending on the corresponding physical models (CNNs) described), then propose the ECM CNN and calculate its bifurcation diagrams, and finally simulate numerically the behaviors of the Chay equations and the ECM CNNs.

\subsection{Theorems}

For a two-port CNN cell, the corresponding local state Eqs. (5) and (6) with "three" state variables assume the following form:

$$
\begin{aligned}
\dot{\mathbf{V}}_{\mathbf{a}} & =\mathbf{A}_{\mathbf{a a}} \mathbf{V}_{\mathbf{a}}+\mathbf{A}_{\mathbf{a b}} \mathbf{V}_{\mathbf{b}}+\mathbf{I}_{\mathbf{a}} \\
\dot{\mathbf{V}}_{\mathbf{b}} & =\mathbf{A}_{\mathbf{b a}} \mathbf{V}_{\mathbf{a}}+\mathbf{A}_{\mathbf{b b}} \mathbf{V}_{\mathbf{b}}
\end{aligned}
$$

where

$$
\begin{gathered}
\mathbf{V}_{\mathbf{a}}=\left[V_{1}, V_{2}\right]^{T}, \quad \mathbf{V}_{\mathbf{b}}=V_{3}, \mathbf{I}_{\mathbf{a}}=\left[I_{1}, I_{2}\right]^{T}, \\
\mathbf{A}_{\mathbf{a a}}=\left[\begin{array}{ll}
a_{11} & a_{12} \\
a_{21} & a_{22}
\end{array}\right], \quad \mathbf{A}_{\mathbf{b b}}=\left[\begin{array}{l}
a_{13} \\
a_{23}
\end{array}\right], \\
\mathbf{A}_{\mathbf{b a}}=\left[\begin{array}{ll}
a_{31} & a_{32}
\end{array}\right], \\
\mathbf{A}_{\mathbf{b b}}=a_{33} .
\end{gathered}
$$

The corresponding CNN cell admittance $\mathbf{Y}_{Q}(s)$ is given by [Chua, 1997]

$$
\begin{aligned}
\mathbf{Y}_{Q}(s) & =\left(s \mathbf{I}-\mathbf{A}_{\mathbf{a a}}\right)-\mathbf{A}_{\mathbf{a b}}\left(s \mathbf{I}-\mathbf{A}_{\mathbf{b b}}\right)^{-1} \mathbf{A}_{\mathbf{b a}} \\
& =\left[\begin{array}{cc}
s-a_{11}-\frac{a_{13} a_{31}}{s-a_{33}} & -a_{12}-\frac{a_{13} a_{32}}{s-a_{33}} \\
-a_{21}-\frac{a_{23} a_{31}}{s-a_{33}} & s-a_{22}-\frac{a_{23} a_{32}}{s-a_{33}}
\end{array}\right] .
\end{aligned}
$$

Now, we introduce a set of theorems for testing the local activity of the CNNs with three state variables and two ports (see [Min \& Yu, 2000] for complete proofs).

Theorem 3.1. $\quad \mathbf{Y}_{Q}(s)$ has a pole in $\operatorname{Re}[s]>0$ if, and only if, $a_{33}>0$, and $\max \left\{\left|a_{13} a_{31}\right|,\left|a_{13} a_{32}\right|\right.$, $\left.\left|a_{23} a_{31}\right|,\left|a_{23} a_{32}\right|\right\} \neq 0$.

Theorem 3.2. Let $b=a_{13} a_{31} a_{33}, c=a_{13} a_{32} a_{33}+$ $a_{23} a_{31} a_{33}, d=a_{13} a_{32}-a_{23} a_{31}, e=a_{23} a_{32} a_{33}$. Then, $\mathbf{Y}_{Q}(s)$ satisfies condition 2 in the Main Theorem if, and only if, at least one of the following conditions holds:

1. $\left(a_{11}+a_{22}\right)>0$.

2. $\left(a_{11}+a_{22}\right) \leq 0, a_{33} \neq 0$, and $\left(a_{11}+a_{22}\right)-$ $\left(a_{13} a_{31}+a_{23} a_{32}\right) / a_{33}>0$.

3. $4 a_{11} a_{22}-\left(a_{12}+a_{21}\right)^{2}<0$.

4. $4\left(b a_{22}+e a_{11}\right)-2 c\left(a_{12}+a_{21}\right)+d^{2} \neq 0$,

$$
\omega^{2}=\frac{2 a_{33}^{2} d^{2}+8 b e-2 c^{2}}{4\left(b a_{22}+e a_{11}\right)-2 c\left(a_{12}+a_{21}\right)+d^{2}}-a_{33}^{2}>0,
$$

$$
\begin{gathered}
4\left[a_{11} a_{22}-\frac{b a_{22}+e a_{11}}{a_{33}^{2}+\omega^{2}}+\frac{b e}{\left(a_{33}^{2}+\omega^{2}\right)^{2}}\right] \\
-\left(a_{12}+a_{21}-\frac{c}{a_{33}^{2}+\omega^{2}}\right)^{2} \\
-\frac{\omega^{2} d^{2}}{\left(a_{33}^{2}+\omega^{2}\right)^{2}}<0 .
\end{gathered}
$$

5. $a_{33} \neq 0$, and $4\left(a_{11} a_{33}^{2}-b\right)\left(a_{22} a_{33}^{2}-e\right)-\left[\left(a_{12}+\right.\right.$ $\left.\left.a_{21}\right) a_{33}^{2}-c\right]^{2}<0$.

6. $a_{33}=0$ and $4 b e-c^{2}<0$.

Theorem 3.3. $\quad \mathbf{Y}_{Q}(s)$ satisfies condition 3 in the Main Theorem if, and only if, at least one of the following conditions holds:

1. If $a_{33}=0, \max \left\{\left|a_{13} a_{31}\right|,\left|a_{13} a_{32}\right|,\left|a_{23} a_{31}\right|\right.$, $\left.\left|a_{23} a_{32}\right|\right\} \neq 0$, and $a_{13} a_{32} \neq a_{23} a_{31}$. 
2. If $a_{33}=0, \max \left\{\left|a_{13} a_{31}\right|,\left|a_{13} a_{32}\right|,\left|a_{23} a_{31}\right|\right.$, $\left.\left|a_{23} a_{32}\right|\right\} \neq 0, a_{13} a_{32}=a_{23} a_{31}$ and

(a) $a_{13} a_{31}+a_{23} a_{32}>0$ or

(b) $a_{13} a_{31} a_{23} a_{32}-a_{13}^{2} a_{32}^{2}<0$.

Theorem 3.4. $\quad \mathbf{Y}_{Q}(s)$ does not have a multiple pole on the imaginary axis.

\subsection{ECM CNN and bifurcation diagram}

The prototype ECM CNN used here is taken from [Chay, 1985], which has three variables, $V, C, n$ :

$$
\begin{aligned}
\dot{V}_{i}= & g_{I}^{*} m_{\infty}^{3} h_{\infty}\left(V_{I}-V_{i}\right)+g_{K, V}^{*} n_{i}^{4}\left(V_{K}-V_{i}\right) \\
& +g_{K, C}^{*} \frac{C_{i}}{1+C_{i}}\left(V_{K}-V_{i}\right)+g_{L}^{*}\left(V_{L}-V_{i}\right) \\
& +D_{1}\left(V_{i+1}+V_{i-1}-2 V_{i}\right) \\
\dot{C}_{i}= & \rho\left[g_{I}^{*} m_{\infty}^{3} h_{\infty}\left(V_{C}-V_{i}\right)-K_{C} C_{i}\right] \\
& +D_{2}\left(C_{i+1}+C_{i-1}-2 C_{i}\right) \\
\dot{n}_{i}= & \frac{n_{\infty}-n_{i}}{\tau_{n}} \\
& i=1,2, \ldots, N
\end{aligned}
$$

where $V_{i}, C_{i}$ and $n_{i}$ are the membrane potential, the intracellular concentration of $\mathrm{Ca}^{2+}$ ions, and the probability of opening the voltage-sensitive $K^{+}$ channel of the $i$ th cell, respectively; $N=30 ; V_{I}$, $V_{k}$ and $V_{L}$ are the reversal potentials for "mixed" $\mathrm{Na}^{+}-\mathrm{Ca}^{2+}, \mathrm{K}^{+}$and leakage ions, respectively; $C$ is the concentration of intracellular $\mathrm{Ca}^{2+}$ ions divided by its dissociation constant from the receptor; $g_{I}^{*}, g_{K, V}^{*}, g_{K, C}^{*}$ and $g_{L}^{*}$ are the maximal conductances divided by the membrane capacitance, where the subscripts $I,(K, V),(K, C)$ and $(L)$ refer to the voltage-sensitive mixed ion channel, voltagesensitive $\mathrm{K}^{+}$channel, $\mathrm{Ca}^{+}$- sensitive $\mathrm{K}^{+}$channel, and the leakage channels, respectively; $m_{\infty}$ and $h_{\infty}$ are the probabilities of activation and inactivation of the mixed channel; $n_{\infty}$ is the steady state value of $n_{i} ; K_{C}$ is the rate constant for the efflux of intracellular $\mathrm{Ca}^{2+}$ ions, $\rho$ is a proportionality constant, and $V_{C}$ the reversal potential for $\mathrm{Ca}^{2+}$ ions; $\tau$ is the relaxation time (in $\mathrm{s}$ ).

The expressions for $m_{\infty}, h_{\infty}, n_{\infty}$ and $\tau_{n}$ (similar to the original Hodgkin-Huxley equations
[Hodgkin \& Huxley, 1952]) are given as follows [Chay, 1985]:

$$
y=\frac{\alpha_{y}}{\alpha_{y}+\beta_{y}},
$$

where $y$ stands for $m_{\infty}, h_{\infty}, n_{\infty}$ and

$$
\begin{aligned}
\alpha_{m} & =\frac{0.1\left(25+V_{i}\right)}{1-\exp \left(-0.1 V_{i}-2.5\right)} \\
\beta m & =4 \exp \left[\frac{-V_{i}-50}{18}\right] \\
\alpha_{h} & =0.07 \exp \left(-0.05 V_{i}-2.5\right) \\
\beta_{h} & =\frac{1}{1+\exp \left(-0.1 V_{i}-2\right)} \\
\alpha_{n} & =\frac{0.01\left(20+V_{i}\right)}{1-\exp \left(-0.1 V_{i}-2\right)} \\
\beta_{n} & =0.125 \exp \left[\frac{-V_{i}-30}{80}\right] \\
\tau_{n} & =\frac{1}{230\left(\alpha_{n}+\beta_{n}\right)} .
\end{aligned}
$$

Furthermore, let the system have periodic conditions: $V_{0}=V_{N}, C_{0}=C_{N}, n_{0}=n_{N}, V_{N+1}=V_{1}$, $C_{N+1}=C_{1}, n_{N+1}=n_{1}$, and let $D_{1}=D_{2}=0.001$, $N=30$ in the numerical simulations.

In a component form, Eqs. (16)-(18) become

$$
\begin{aligned}
\dot{\mathbf{V}} & =f_{1}(\mathbf{V}, \mathbf{C}, \mathbf{n})+\mathbf{D}_{\mathbf{1}} \nabla^{2} \mathbf{V} \\
\dot{\mathbf{C}} & =f_{2}(\mathbf{V}, \mathbf{C}, \mathbf{n})+\mathbf{D}_{\mathbf{1}} \nabla^{\mathbf{2}} \mathbf{C} \\
\dot{\mathbf{n}} & =f_{3}(\mathbf{V}, \mathbf{C}, \mathbf{n})
\end{aligned}
$$

where $\nabla^{2}$ corresponds to an $N \times N$ matrix.

The "cell equilibrium points" $Q_{i}$ 's of Eqs. (27)-(29) for the restricted local activity domain [Dogaru, 1998] can be determined via

$$
\begin{aligned}
& f_{1}(\mathbf{V}, \mathbf{C}, \mathbf{n})=0 \\
& f_{2}(\mathbf{V}, \mathbf{C}, \mathbf{n})=0 \\
& f_{3}(\mathbf{V}, \mathbf{C}, \mathbf{n})=0 .
\end{aligned}
$$

Numerical calculation shows that Eqs. (30)-(32) have a cell equilibrium point $Q$. 
The cell coefficients $a_{m, n}(Q)^{\prime} s$ are defined via the corresponding Jacobian

$$
\begin{aligned}
\mathbf{A} & \triangleq\left[\begin{array}{lll}
a_{11}(Q) & a_{12}(Q) & a_{13}(Q) \\
a_{21}(Q) & a_{22}(Q) & a_{23}(Q) \\
a_{31}(Q) & a_{32}(Q) & a_{33}(Q)
\end{array}\right] \\
& \triangleq\left[\begin{array}{lll}
\frac{\partial f_{1}(\mathbf{U})}{\partial \mathbf{V}} & \frac{\partial f_{1}(\mathbf{U})}{\partial \mathbf{C}} & \frac{\partial f_{1}(\mathbf{U})}{\partial \mathbf{n}} \\
\frac{\partial f_{2}(\mathbf{U})}{\partial \mathbf{V}} & \frac{\partial f_{2}(\mathbf{U})}{\partial \mathbf{C}} & \frac{\partial f_{2}(\mathbf{U})}{\partial \mathbf{n}} \\
\frac{\partial f_{3}(\mathbf{U})}{\partial \mathbf{V}} & \frac{\partial f_{3}(\mathbf{U})}{\partial \mathbf{C}} & \frac{\partial f_{3}(\mathbf{U})}{\partial \mathbf{n}}
\end{array}\right] \\
& \triangleq\left[\begin{array}{lll}
\mathbf{A}_{\mathbf{a a}} & \mathbf{A}_{\mathbf{a b}} \\
\mathbf{A}_{\mathbf{b a}} & \mathbf{A}_{\mathbf{b b}}
\end{array}\right],
\end{aligned}
$$

where $\mathbf{U}=(\mathbf{V}(Q), \mathbf{C}(Q), \mathbf{n}(Q))$. Consequently, the corresponding $\mathrm{CNN}$ cell admittance $\mathbf{Y}_{Q}(s)$ can be calculated via (33) and (15).

Using the Main Theorem and Theorems 3.13.4 , the locally active and unstable domain, locally passive domain, and edge of chaos with respect to the equilibrium points $Q^{\prime} s$ (i.e. the bifurcation diagrams of the ECM CNNs) can be numerically calculated via computer programs.

Now, let the parameters $g_{K, C}^{*}$ and $g_{K, V}^{*}$ vary in the intervals $[5,15]$ and $[200,2200]$, respectively, and the other parameters are fixed. Then, the calculation results are shown in Fig. 1. It follows that

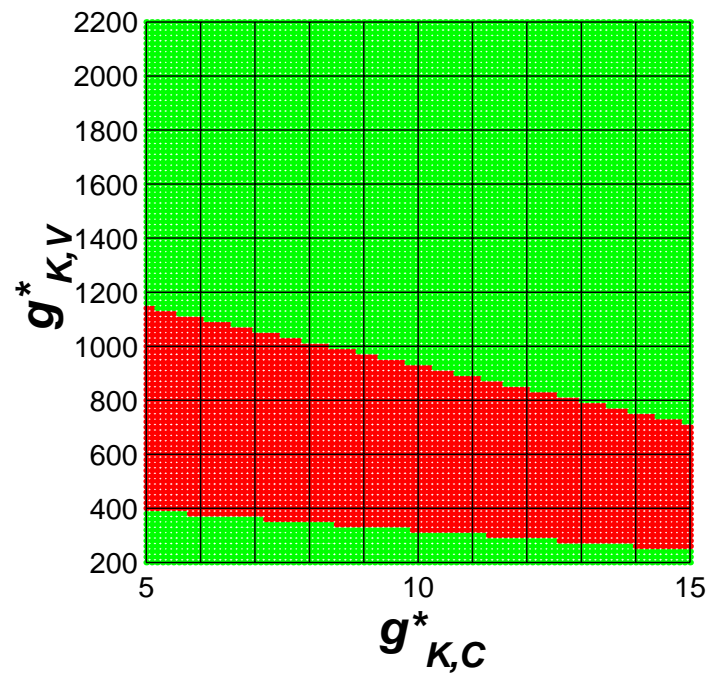

Fig. 1. Bifurcation diagram of the coupled excitable cell model CNN at cross-section $g_{\mathrm{K}, \mathrm{C}}^{*} \in[5,15]$ and $g_{\mathrm{K}, \mathrm{V}}^{*} \in[200$, 2200]. The domains are coded as follows: edge of chaos (locally active and stable) domain (red), locally active and unstable domain (green). for the parameter groups:

- The bifurcation diagram with respect to the equilibrium point $Q^{\prime} s$ has two locally active and unstable domains separated by an edge of chaos. The numerical simulations will show that nearby the edge of chaos, chaotic trajectories and/or complex periodic trajectories may emerge.

\subsection{Simulations of the ECM CNN Dynamics}

Now, we simulate numerically the three-dimensional (3D) ECM equations and the ECM CNNs with different cell parameters based on the bifurcation graph shown in Fig. 1. The calculated results are shown in Table 1 and some of the simulation graphs are shown in Figs. 2-11, respectively. Our numerical simulations demonstrate that

- The qualitative behaviors of the 3D ECM equations and the corresponding ECM CNNs may be similar (see Figs. 2, 6 and 9) or different (see Figs. 3-5, 7, 8, 10 and 11), depending on the locations of selected cell parameters and the coupling parameters. This may imply that 3D ECMs cannot always describe correctly the dynamics of the practical ECM CNNs.

- In fact, the oscillation of the "normal heartbeat" is robust (see Fig. 2 and parameter group No. 1 listed in Table 1). However, the frequencies of the "heartbeats" of the 3D ECM and the corresponding ECM CNN are about 70 beats/min and 60 beats/min, respectively.

- The dynamic pattern of the 3D ECM with parameter group No. 3 listed in Table 1 shows chaotic characteristics [Fig. 4(a)]. However, the corresponding behaviors of the ECM CNN converges to a 10-periodic limit cycle [Figs. 4(b)$4(\mathrm{e})]$. It is observed that if the coupling parameters $D_{1}=D_{2}=0.0001$, the graphs of the time evolution of the corresponding ECM CNN will also display chaotic patterns.

- The dynamic patterns of ECM CNNs with the parameter groups Nos. 1-12 seem to exhibit the processes of the heartbeat changes of the patients:

Normal heart rate $\rightarrow$ Multi-period heart rate

$\rightarrow$ Chaotic heart rate $\rightarrow$ Multi-period heart rate $\rightarrow$ Stop beating.

This fact implies that controlling parameters $g_{K, V}^{*}$ and $g_{K, C}^{*}$ might make a heart with 
Table 1. Cell parameters and corresponding dynamic properties of the 3D ECMs and the ECM CNNs, where the parameter group $\left\{V_{k}, V_{I}, V_{L}, V_{C}, V_{n}, V_{m}, g_{I}^{*}, g_{L}^{*}, K_{C}, \rho\right\}=\{-75 \mathrm{mV}, 100 \mathrm{mV},-40 \mathrm{mV}, 100 \mathrm{mV},-30 \mathrm{mV},-50 \mathrm{mV}$, $\left.1800 \mathrm{~s}^{-1}, 7 \mathrm{~s}^{-1}, 3.3 / 18 \mathrm{mV}, 0.27 \mathrm{mV}^{-1} \mathrm{~s}^{-1}\right\}$. For items $1-5$ and 11 , take parameters $D_{1}=D_{2}=0.001$; for items 6-10 and 12 , take parameters $D_{1}=D_{2}=0.0001$. The symbols $\Downarrow, j p, \oplus, F S$ indicate that convergent patterns, $j$-period patterns, chaotic patterns and complex period patterns are observed near to or far from the corresponding equilibrium points, respectively. The numbers marked by a $*$ indicate that the corresponding cell parameters lie on the edge of the chaos domain.

\begin{tabular}{ccccccc}
\hline No. & $g_{K, C}^{*} / \mathrm{s}^{-1}$ & $g_{K, V}^{*} / \mathrm{s}^{-1}$ & Cell Equilibrium Point & Eigenvalue & Pattern (3D\&HD) \\
\hline 1 & 10 & 1700 & $-32.4050,2.4575,0.2816$ & $-0.0152,11.9125 \pm 33.0239 \mathrm{i}$ & $1 \mathrm{p}$ & $1 \mathrm{p}$ \\
2 & 10 & 1600 & $-31.7282,2.6428,0.2916$ & $-0.0113,11.7799 \pm 37.4446 \mathrm{i}$ & $2 \mathrm{p}$ & $9 \mathrm{p}$ \\
3 & 10 & 1570 & $-31.5245,2.6989,0.2946$ & $-0.0104,11.6724 \pm 38.7552 \mathrm{i}$ & $\oplus$ & $10 \mathrm{p}$ \\
4 & 10 & 1400 & $-30.3556,3.0213,0.3122$ & $-0.0067,10.4284 \pm 46.0900 \mathrm{i}$ & $18 \mathrm{p}$ & $17 \mathrm{p}$ \\
5 & 10 & 1000 & $-27.3547,3.8065,0.3589$ & $-0.0025,2.3721 \pm 62.7407 \mathrm{i}$ & FS & FS \\
6 & 11 & 1000 & $-27.7697,3.7049,0.3523$ & $-0.0034,3.8788 \pm 58.2799 \mathrm{i}$ & FS & FS \\
7 & 11 & 1400 & $-30.9635,2.8538,0.3030$ & $-0.0107,11.2109 \pm-38.9826 \mathrm{i}$ & FS & $14 \mathrm{p}$ \\
8 & 11 & 1700 & $-33.3057,2.2152,0.2685$ & $-0.0370,11.5858 \pm-21.8000 \mathrm{i}$ & $\oplus$ & $\oplus$ \\
9 & 11 & 1735 & $-33.5929,2.1394,0.2643$ & $-0.0460,11.3675 \pm 19.3632 \mathrm{i}$ & $3 \mathrm{p}$ & $3 \mathrm{p}$ \\
10 & 11 & 2200 & $-38.1796,1.0962,0.2029$ & $23.5628,-18.8322,0.0711$ & $1 \mathrm{p}$ & $2 \mathrm{p}$ \\
$11^{*}$ & 10 & 920 & $-26.6775,3.9656,0.3696$ & $-0.0021,-0.3222 \pm 65.8716 \mathrm{i}$ & $\Downarrow$ & $\Downarrow$ \\
$12^{*}$ & 11 & 880 & $-26.7034,3.9596,0.3692$ & $-0.0025,-0.2139 \pm 63.5175 \mathrm{i}$ & $\Downarrow$ & $\Downarrow$ \\
\hline
\end{tabular}

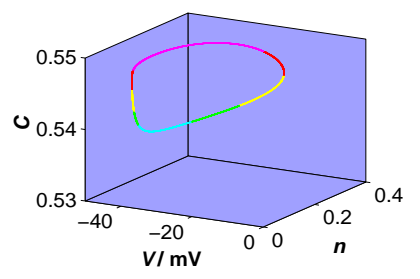

(a)

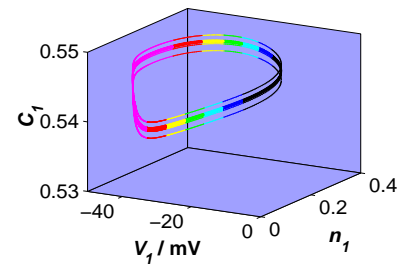

(b)

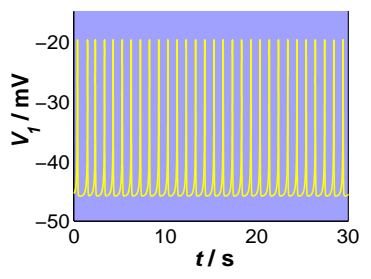

(c)

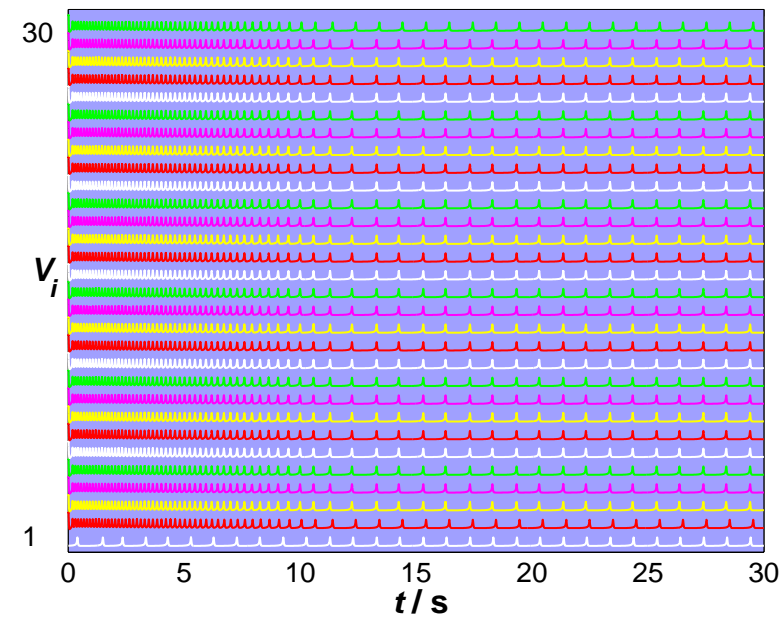

(d)

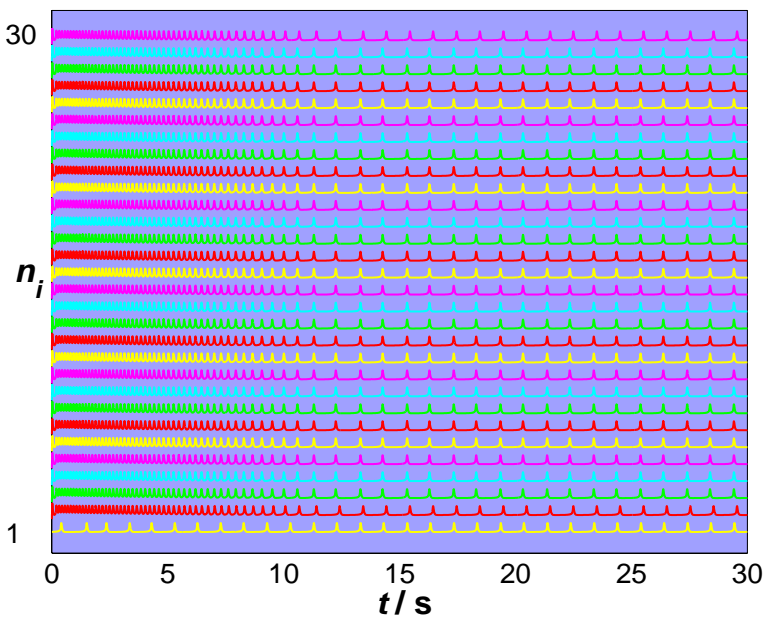

(e)

Fig. 2. Graphs generated by the parameter group No. 1 listed in Table 1. (a) The one-period trajectory of the state variables $V, C$ and $n$ of the ECM equations initial conditions: $(V(0), C(0), n(0))=(-45.4357,0.5412,0.1247)$. (b) The trajectory of the state variables $V_{1}, C_{1}$ and $n_{1}$ of the ECM CNN converges to a period-1 limit cycle. (c) The time evolution of the state variables $V_{1}$ of the ECM CNN. (d) and (e) The graphs of the time evolution of the state variables $V_{i}^{\prime} s$ and $n_{i}^{\prime} s$ of the ECM CNN; initial conditions: $\left(V_{1}(0), C_{1}(0), n_{1}(0)\right)=(V(0), C(0), n(0))$ and $\left(V_{i}(0), C_{i}(0), n_{i}(0)\right)=(0,0,0), i=2,3, \ldots, 30$. 
disordered heartbeat to recover the normal frequency of heartbeat.

- The dynamics of the first cell (pacemaker cell) of an ECM CNN may be quite different from other ones. In fact, Figs. 10 and 11 demonstrate that the first cell shows period-3 and period- 2 trajectories but the other 29 cells display period-21 and period-18 trajectories, respectively.

\subsection{Concluding remarks}

Coupled nonlinear dynamical systems (CNDS) have been widely studied in the recent years. However, the dynamical properties of the CNNs are difficult to study, particularly for ECMs. The local activity theory of CNNs provides a new tool for the study of the ECMs.

Our numerical simulations imply that:

(a) Regulating parameters $g_{K, V}^{*}$ and $g_{K, C}^{*}$ might make a disordered heart to recover the normal frequency of heartbeat.

(b) 3D ECMs may not always describe correctly the dynamics of the corresponding HD ECM CNNs. (c) The dynamics of the pacemaker cell of an ECM CNN may be quite different from the others.

(d) The values of the diffusion coefficients $D_{1}, D_{2}$ may make the dynamic behaviors of ECMs considerably different. Experimental data on the diffusion coefficients are expected for computer modeling.

(e) No sudden death phenomena are simulated, which are quite different from the simulation results on the Hodgkin-Huxley CNN [Min et al., $2000 \mathrm{~b}]$.

Our research shows the effectiveness of the local activity theory in the study of coupled excitable cell models.

\section{Analytical Criteria for Local Activity of CNN with Four State Variables and Two Ports}

In the following subsections, first, we will set up some analytical criteria for the Main Theorem for a

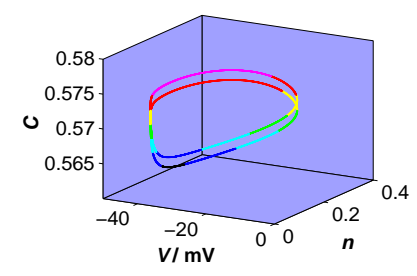

(a)

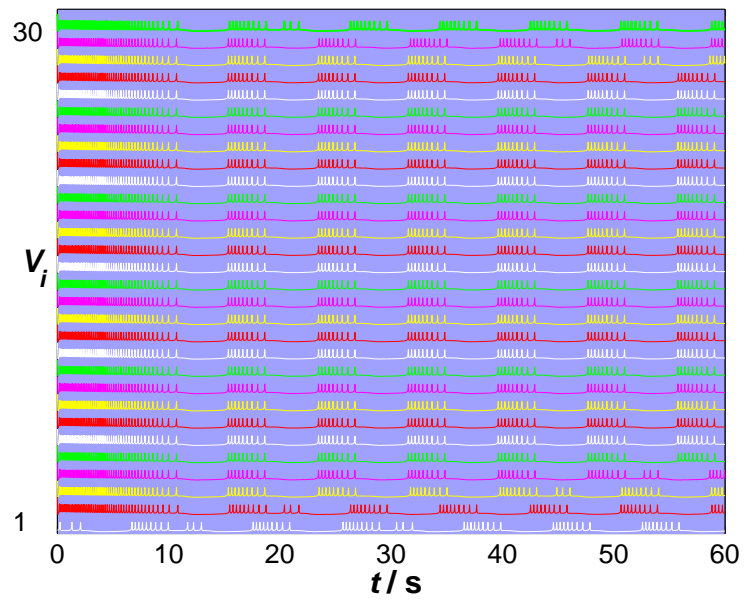

(d)

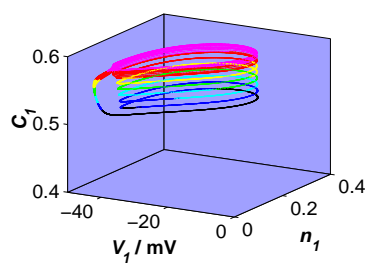

(b)

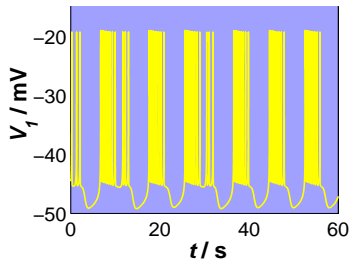

(c)

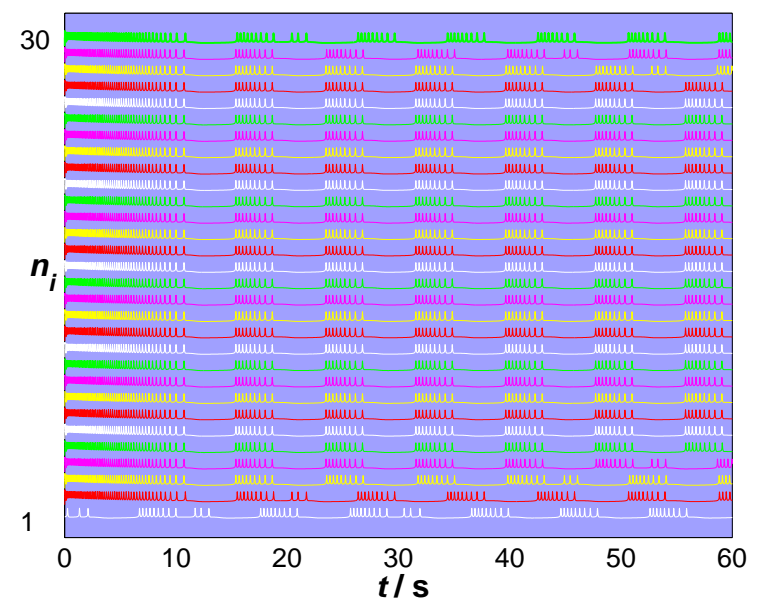

(e)

Fig. 3. Graphs generated by the parameter group No. 2 listed in Table 1. (a) The period-2 trajectory of the state variables $V, C$ and $n$ of the ECM equations; initial conditions: $(V(0), C(0), n(0))=(-44.5339,0.5640,0.1321)$. (b) The trajectory of the state variables $V_{1}, C_{1}$ and $n_{1}$ of the ECM CNN converges to a period-9 limit cycle. (c) The time evolution of the state variables $V_{1}$ of the ECM CNN. (d) and (e) The graphs of the time evolution of the state variables $V_{i}^{\prime} s$ and $n_{i}^{\prime} s$ of the ECM CNN; initial conditions: $\left(V_{1}(0), C_{1}(0), n_{1}(0)\right)=(V(0), C(0), n(0))$ and $\left(V_{i}(0), C_{i}(0), n_{i}(0)\right)=(0,0,0), i=2,3, \ldots, 30$. 


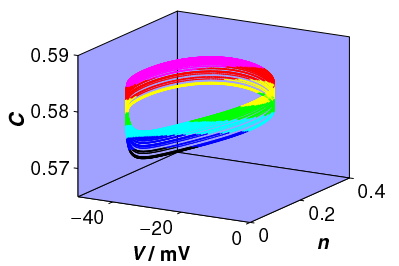

(a)

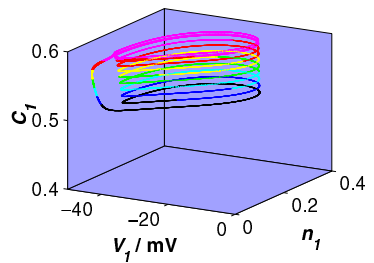

(b)

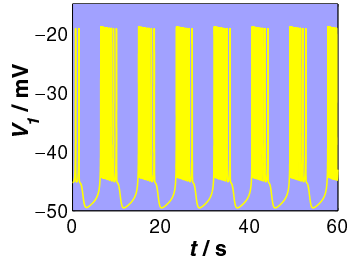

(c)

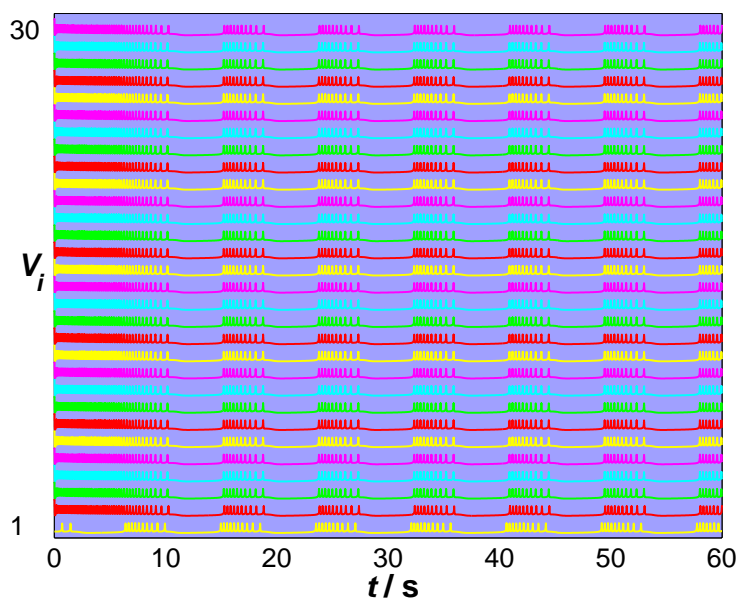

(d)

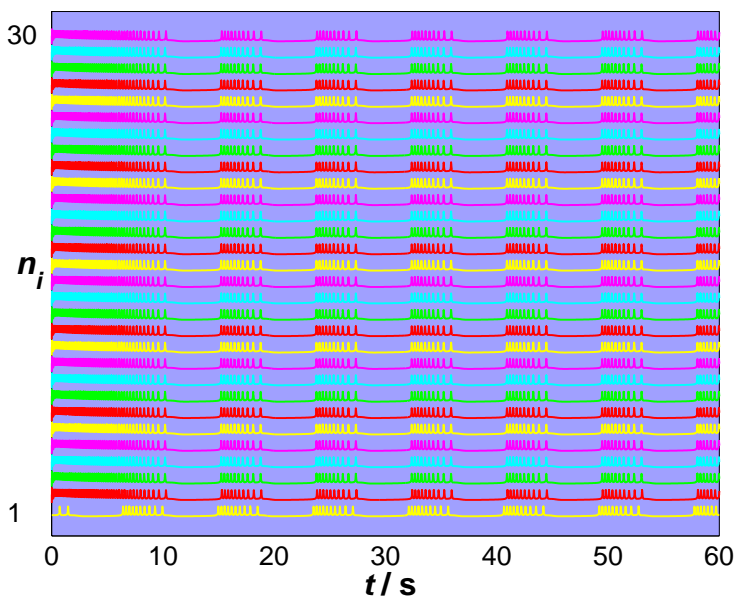

(e)

Fig. 4. Graphs generated by the parameter group No. 3 listed in Table 1. (a) The chaotic trajectory of the state variables $V, C$ and $n$ of the ECM equations; initial conditions: $(V(0), C(0), n(0))=(-45.2256,0.5785,0.1271)$. (b) The trajectory of the state variables $V_{1}, C_{1}$ and $n_{1}$ of the ECM CNN converges to a ten-period limit cycle. (c) The time evolution of the state variables $V_{1}$ of the ECM CNN. (d) and (e) The graphs of the time evolution of the state variables $V_{i}^{\prime} s$ and $n_{i}^{\prime} s$ of the ECM CNN; initial conditions: $\left(V_{1}(0), C_{1}(0), n_{1}(0)\right)=(V(0), C(0), n(0))$, and $\left(V_{i}(0), C_{i}(0), n_{i}(0)\right)=(0,0,0), i=2,3, \ldots, 30$.

general two-port CNN cell with four state variables. Second, we will calculate the bifurcation diagram of the SCC CNN with two reaction-diffusion coefficients and then simulate numerically the dynamic behaviors of the 4D SCCs and the SCC CNNs.

\subsection{Theorems}

For a two-port CNN cell with four state variables, the corresponding local state equations assume the following form:

$$
\begin{aligned}
\dot{\mathbf{V}}_{\mathbf{a}} & =\mathbf{A}_{\mathbf{a a}} \mathbf{V}_{\mathbf{a}}+\mathbf{A}_{\mathbf{a b}} \mathbf{V}_{\mathbf{b}}+\mathbf{I}_{\mathbf{a}} \\
\dot{\mathbf{V}}_{\mathbf{b}} & =\mathbf{A}_{\mathbf{b a}} \mathbf{V}_{\mathbf{a}}+\mathbf{A}_{\mathbf{b b}} \mathbf{V}_{\mathbf{b}},
\end{aligned}
$$

where

$$
\begin{aligned}
& \mathbf{V}_{\mathbf{a}}=\left[V_{1}, V_{2}\right]^{T}, \mathbf{V}_{\mathbf{b}}=\left[V_{3}, V_{4}\right]^{T}, \mathbf{I}_{\mathbf{a}}=\left[I_{1}, I_{2}\right]^{T}, \\
& \mathbf{A}_{\mathbf{a a}}=\left[\begin{array}{ll}
a_{11} & a_{12} \\
a_{21} & a_{22}
\end{array}\right], \quad \mathbf{A}_{\mathbf{a b}}=\left[\begin{array}{ll}
a_{13} & a_{14} \\
a_{23} & a_{24}
\end{array}\right], \\
& \mathbf{A}_{\mathbf{b a}}=\left[\begin{array}{ll}
a_{31} & a_{32} \\
a_{41} & a_{42}
\end{array}\right], \quad \mathbf{A}_{\mathbf{b b}}=\left[\begin{array}{ll}
a_{33} & a_{34} \\
a_{43} & a_{44}
\end{array}\right] .
\end{aligned}
$$

The corresponding CNN cell admittance $Y_{Q}(s)$ is given by

$$
\begin{aligned}
\mathbf{Y}_{Q}(s)= & \left(s \mathbf{I}-\mathbf{A}_{\mathbf{a a}}\right)-\mathbf{A}_{\mathbf{a b}}\left(s \mathbf{I}-\mathbf{A}_{\mathbf{b b}}\right)^{-1} \mathbf{A}_{\mathbf{b a}} \\
= & {\left[\begin{array}{cc}
s-a_{11} & -a_{12} \\
-a_{21} & s-a_{22}
\end{array}\right]-\frac{1}{s^{2}+a_{00} s+b_{00}} } \\
& \times\left[\begin{array}{cc}
q_{11} s+q_{110} & q_{12} s+q_{120} \\
q_{13} s+q_{130} & q_{14} s+q_{140}
\end{array}\right]
\end{aligned}
$$

where

$$
\begin{aligned}
q_{11}= & a_{13} a_{31}+a_{14} a_{41} \\
q_{110}= & a_{13} a_{34} a_{41}+a_{14} a_{43} a_{31} \\
& -a_{13} a_{31} a_{44}-a_{41} a_{14} a_{33} \\
q_{12}= & a_{13} a_{32}+a_{14} a_{42} \\
q_{120}= & a_{13} a_{34} a_{42}+a_{14} a_{32} a_{43} \\
& -a_{13} a_{32} a_{44}-a_{14} a_{33} a_{42} \\
q_{13}= & a_{23} a_{31}+a_{24} a_{41}
\end{aligned}
$$




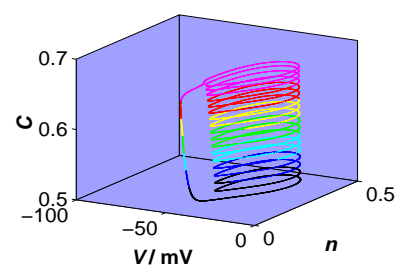

(a)

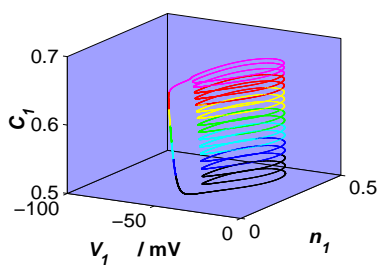

(b)

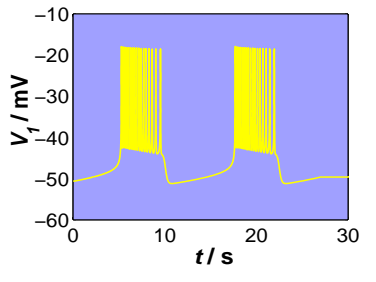

(c)

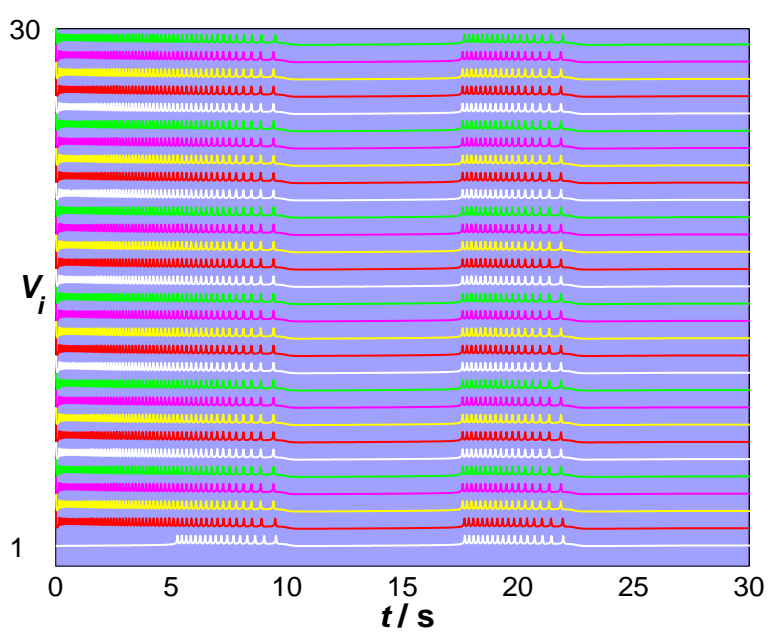

(d)

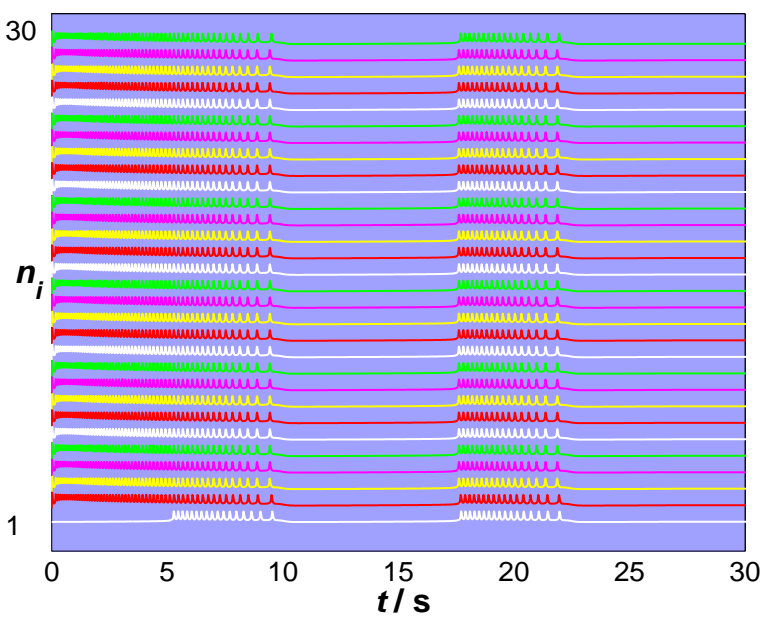

(e)

Fig. 5. Graphs generated by the parameter group No. 4 listed in Table 1. (a) The period-18 trajectory of the state variables $V, C$ and $n$ of the ECM equations; initial conditions: $(V(0), C(0), n(0))=(-50.6499,0.6083,0.0848)$. (b) The trajectory of the state variables $V_{1}, C_{1}$ and $n_{1}$ of the ECM CNN converges to a period-17 limit cycle. (c) The time evolution of the state variables $V_{1}$ of the ECM CNN. (d) and (e) The graphs of the time evolution of the state variables $V_{i}^{\prime} s$ and $n_{i}^{\prime} s$ of the ECM CNN; initial conditions: $\left(V_{1}(0), C_{1}(0), n_{1}(0)\right)=(V(0), C(0), n(0))$, and $\left(V_{i}(0), C_{i}(0), n_{i}(0)\right)=(0,0,0), i=2,3, \ldots, 30$.

$$
\begin{aligned}
q_{130}= & a_{24} a_{31} a_{43}+a_{23} a_{34} a_{41} \\
& -a_{23} a_{31} a_{44}-a_{24} a_{33} a_{41} \\
q_{14}= & a_{23} a_{32}+a_{24} a_{42}, \\
q_{140}= & a_{23} a_{34} a_{42}+a_{24} a_{32} a_{43} \\
& -a_{23} a_{32} a_{44}-a_{24} a_{33} a_{42} \\
a_{00}= & -a_{33}-a_{44} \\
b_{00}= & a_{33} a_{44}-a_{34} a_{43} .
\end{aligned}
$$

Lemma 4.1. Let $p(s)=s^{2}+a s+b$. Then $\exists s \in \mathbb{C}$, and $\operatorname{Re}(s)>0$, s.t. $p(s)=0 \Leftrightarrow(a, b) \in A=$ $\{(x, y) \mid x<0$, or $y<0\}$.

Proof. Since $p(s)=0 \Rightarrow s=\left(-a \pm \sqrt{a^{2}-4 b}\right) / 2$, it implies that

(a) If $a \geq 0$ and $b \geq 0$, then $\operatorname{Re}(s) \leq 0$.

(b) If $a<0$ or $b<0$, then there exists at least a root $s$ of $p(s)=0$ s.t. $\operatorname{Re}(s)>0$.

This completes the proof.
Lemma 4.1 gives the following result.

Theorem 4.1. For $i=1,2,3,4$, let $q_{1 i}, q_{1 i 0}, a_{00}$ and $b_{00}$ be defined by formulas (36)-(45). Then, $\mathbf{Y}_{Q}(s)$ satisfies Condition 1 in the Main Theorem if, and only if, at least one of the following conditions holds: there exists an $i \in\{1,2,3,4\}$ s.t.

1. If two roots of $s^{2}+a_{00} s+b_{00}=0$ do not equal $-q_{1 i 0} / q_{1 i}$, and

$$
\left(a_{00}, b_{00}\right) \in A=\{(x, y) \mid x<0 \text { or } y<0\} .
$$

2. If a root $s_{1}$ of $s^{2}+a_{00} s+b_{00}=0$ equals $-q_{1 i 0} / q_{1 i}$, but another root $s_{2}$ must satisfy $\operatorname{Re}\left(s_{2}\right)>0$.

Proof. By Lemma 4.1, Condition 1 or Condition 2 guarantees that $\mathbf{Y}_{Q}(s)$ has a pole in $\operatorname{Re}(s)>0$.

Theorem 4.2. For $i=1,2,3$, 4, let $q_{1 i}, q_{1 i 0}, a_{00}$ and $b_{00}$ be defined by formulas (36)-(45). Then, $\mathbf{Y}_{Q}(s)$ satisfies Condition 3 in the Main Theorem if, and only if, at least one of the following 


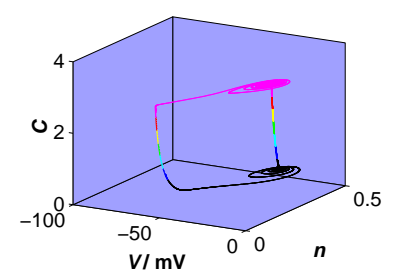

(a)

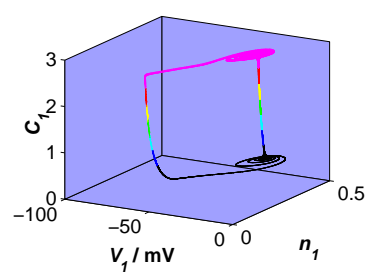

(b)

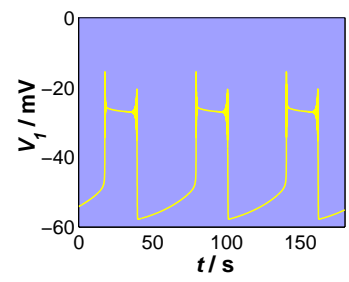

(c)

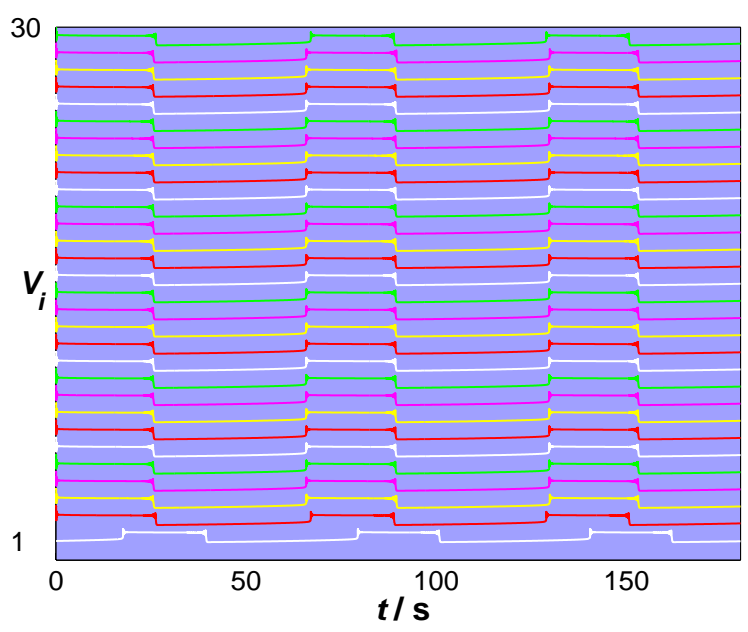

(d)

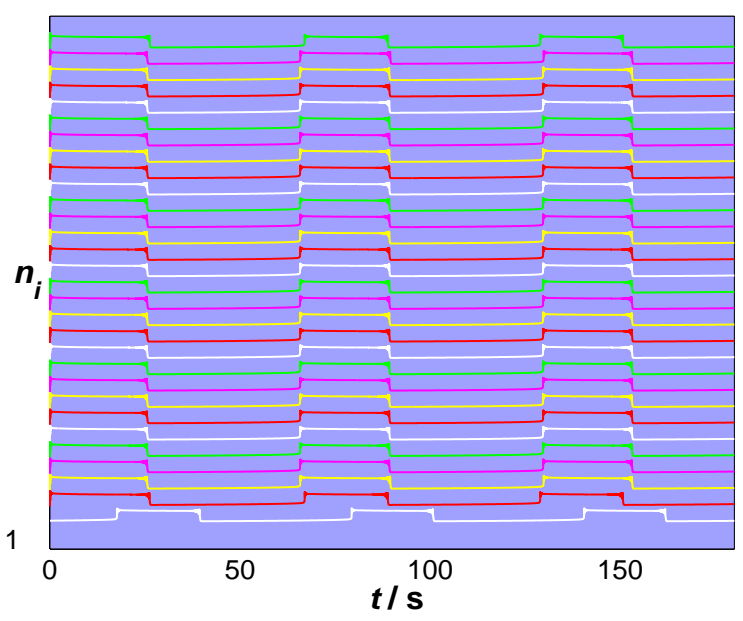

(e)

Fig. 6. Graphs generated by the parameter group No. 5 listed in Table 1. (a) The FS period trajectory of the state variables $V, C$ and $n$ of the ECM equations; initial conditions: $(V(0), C(0), n(0))=(-54.1528,1.0179,0.0642)$. (b) The trajectory of the state variables $V_{1}, C_{1}$ and $n_{1}$ of the ECM CNN converges to a FS period trajectory. (c) The time evolution of the state variables $V_{1}$ of the ECM CNN. (d) and (e) The graphs of the time evolution of the state variables $V_{i}^{\prime} s$ and $n_{i}^{\prime} s$ of the ECM CNN; initial conditions: $\left(V_{1}(0), C_{1}(0), n_{1}(0)\right)=(V(0), C(0), n(0))$ and $\left(V_{i}(0), C_{i}(0), n_{i}(0)\right)=(0,0,0), i=2,3, \ldots, 30$.

\section{conditions holds:}

1. If $a_{00} \neq 0, b_{00}=0, \max \left\{\left|q_{110}\right|,\left|q_{120}\right|,\left|q_{130}\right|\right.$, $\left.\left|q_{140}\right|\right\} \neq 0$, and
(a) $q_{120} \neq q_{130}$, or
(b) $q_{120}=q_{130}$ but $\left(q_{110} q_{140}-q_{120} q_{130}\right) / a_{00}>0$, or $q_{1 i 0} / a_{00}>0, i=1$, or, 2 or, 4 .

2. If $a_{00}=0, b_{00}>0, \max \left\{\left|q_{11}\right|+\left|q_{110}\right|,\left|q_{12}\right|+\right.$ $\left.\left|q_{120}\right|,\left|q_{13}\right|+\left|q_{130}\right|,\left|q_{14}\right|+\left|q_{140}\right|\right\} \neq 0$, and

(a) $\mathbf{Y}_{Q}(s)$ does not satisfy the following condition

(*) $q_{110}=q_{140}=0, q_{12}=q_{13}, q_{120}=q_{130}$, or

(b) $\mathbf{Y}_{Q}(s)$ satisfies the condition $(*)$ and $q_{11}+q_{14}>0$ or $q_{11} q_{14}-q_{12}^{2}-q_{120}^{2} / b_{00}<0$.

Proof. The expression of $\mathbf{Y}_{Q}(s)$ has the form

$$
\begin{aligned}
\mathbf{Y}_{Q}(s)= & {\left[\begin{array}{cc}
s-a_{11} & -a_{12} \\
-a_{21} & s-a_{22}
\end{array}\right]-\frac{1}{s^{2}-a_{00} s+b_{00}} } \\
& \times\left[\begin{array}{cc}
q_{11} s+q_{110} & q_{12} s+q_{120} \\
q_{13} s+q_{130} & q_{14} s+q_{140}
\end{array}\right]
\end{aligned}
$$

1. From Eq. (46), if $\mathbf{Y}_{Q}(s)$ has a simple pole $s=0$ on the imaginary axis, then $a_{00} \neq 0, b_{00}=0$ and

$$
\max \left\{\left|q_{110}\right|,\left|q_{120}\right|,\left|q_{130}\right|,\left|q_{140}\right|\right\} \neq 0 .
$$

In this case,

$$
k_{1}=\lim _{s \rightarrow 0} s Y_{Q}(s)=-\frac{1}{a_{00}}\left[\begin{array}{ll}
q_{110} & q_{120} \\
q_{130} & q_{140}
\end{array}\right] .
$$

Therefore,

(a) If $q_{130} \neq q_{120}$, then $k_{1}$ is not a Hermitian.

(b) If $q_{130}=q_{120}$, and $\left(q_{110} q_{140}-q_{120} q_{130}\right) / a_{00}>0$, or $q_{1 i 0} / a_{00}>0, i=1$, or, 2 or, 4 . Then $k_{1}$ is not a positive semi-definite Hermitian matrix. This shows that Condition 1 in the Theorem is satisfied.

2. Equation (46) implies that if $\mathbf{Y}_{Q}(s)$ has a simple pole $s=i \omega$ on the imaginary axis, then

$$
\left\{\begin{array}{l}
a_{00}=0, b_{00}>0 \text { and } \\
\max \left\{\left|q_{11}\right|+\left|q_{110}\right|,\left|q_{12}\right|+\left|q_{120}\right|,\left|q_{13}\right|\right. \\
\left.\quad+\left|q_{130}\right|,\left|q_{14}\right|+\left|q_{140}\right|\right\} \neq 0 .
\end{array}\right.
$$




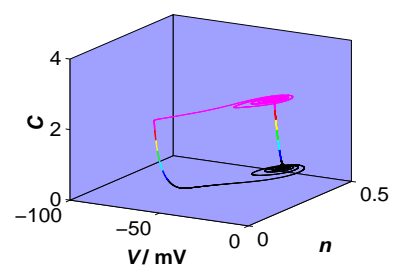

(a)

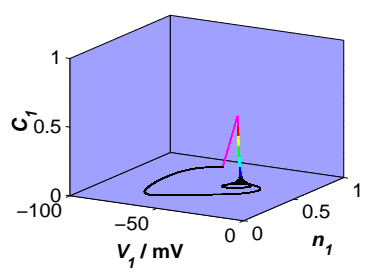

(b)

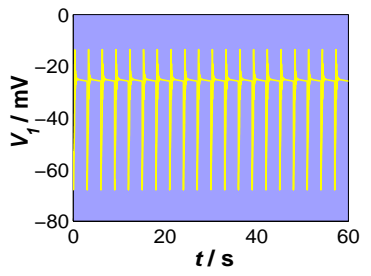

(c)

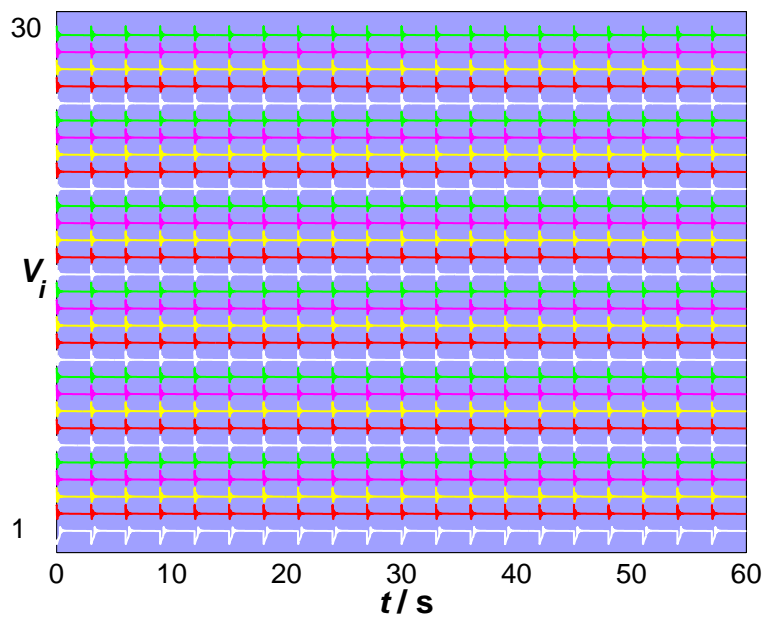

(d)

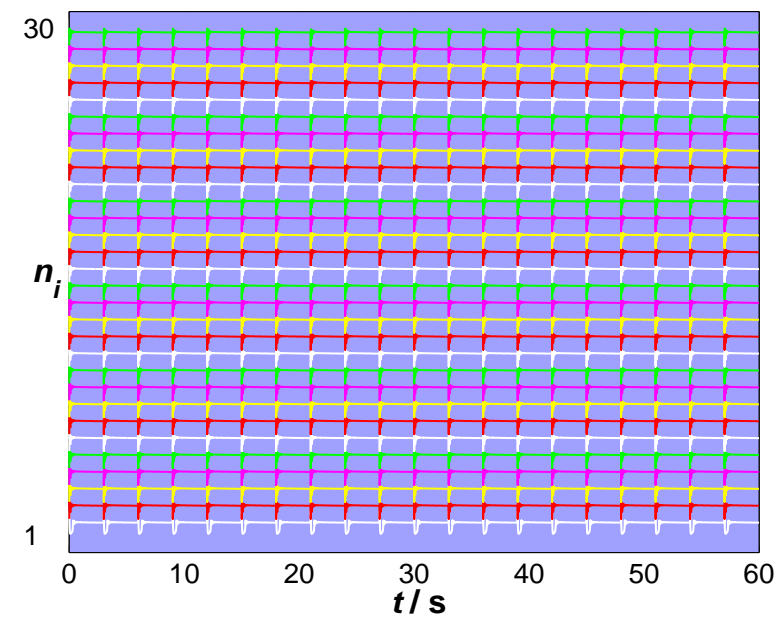

(e)

Fig. 7. Graphs generated by the parameter group No. 6 listed in Table 1. (a) The FS period trajectory of the state variables $V, C$ and $n$ of the ECM equations; initial conditions: $(V(0), C(0), n(0))=(-53.5931,0.7699,0.0672)$. (b) The trajectory of the state variables $V_{1}, C_{1}$ and $n_{1}$ of the ECM CNN converges to a FS period trajectory. (c) The time evolution of the state variables $V_{1}$ of the ECM CNN. (d) and (e) The graphs of the time evolution of the state variables $V_{i}^{\prime} s$ and $n_{i}^{\prime} s$ of the ECM CNN; initial conditions: $\left(V_{1}(0), C_{1}(0), n_{1}(0)\right)=(-53.0990,0.0699,0.7125)$, and $\left(V_{i}(0), C_{i}(0), n_{i}(0)\right)=(0,0,0)$, $i=2,3, \ldots, 30$.

In this case,

$$
\begin{aligned}
k_{1} & =\lim _{s \rightarrow i \sqrt{b_{00}}}\left(s-i \sqrt{b_{00}}\right) \mathbf{Y}_{Q}(s) \\
& =\frac{-1}{2 i \sqrt{b_{00}}}\left[\begin{array}{ll}
q_{11} i \sqrt{b_{00}}+q_{110} & q_{12} i \sqrt{b_{00}}+q_{120} \\
q_{13} i \sqrt{b_{00}}+q_{130} & q_{14} i \sqrt{b_{00}}+q_{140}
\end{array}\right] \\
& =\frac{-1}{2}\left[\begin{array}{ll}
q_{11}-i q_{110} / \sqrt{b_{00}} & q_{12}-i q_{120} / \sqrt{b_{00}} \\
q_{13}-i q_{130} / \sqrt{b_{00}} & q_{14}-i q_{140} / \sqrt{b_{00}}
\end{array}\right] .
\end{aligned}
$$

Therefore,

(a) If Condition $(*)$ does not hold, then $k_{1}$ is not a Hermitian matrix.

(b) If Condition $(*)$ holds, then

$$
\begin{aligned}
\operatorname{det}\left|\lambda I-k_{1}\right|= & \lambda^{2}+\lambda\left(q_{11}+q_{14}\right) / 2+q_{11} a_{14} / 4 \\
& -\left(q_{12} / 2\right)^{2}-q_{120}^{2} /\left(4 b_{00}\right) .
\end{aligned}
$$

Hence, if $\left(q_{11}+q_{14}\right)>0$ or $q_{11} q_{14} / 4-\left(q_{12} / 2\right)^{2}-$ $q_{120}^{2} /\left(4 b_{00}\right)<0$, then $k_{1}$ is not a positive semidefinite Hermitian matrix. In the case that the pole $s=-i \sqrt{b_{00}}$, the same conclusion can also be obtained. Finally,

$$
k_{1}=\lim _{\omega_{\rho} \rightarrow \infty} \mathbf{Y}_{Q}\left(i \omega_{\rho}\right) / i \omega_{\rho}=I
$$

is a positive definite matrix.

Thus, we have completed the proof.

In order to set up the analytic criteria for Condition 2 in the Main Theorem, the following equalities are stated in advance:

$$
\begin{aligned}
A_{11} & =2\left(a_{00} q_{11}-q_{110}\right) ; B_{11}=2 b_{00} q_{110} \\
A_{12} & =a_{00}\left(q_{13}+q_{12}\right)-q_{130}-q_{120} \\
B_{12} & =b_{00}\left(q_{120}+q_{130}\right) \\
B_{120} & =b_{00}\left(q_{12}-q_{13}\right)+a_{00}\left(q_{130}-q_{120}\right) \\
A_{14} & =2\left(a_{00} q_{14}-q_{140}\right) \\
B_{14} & =2 b_{00} q_{140}
\end{aligned}
$$




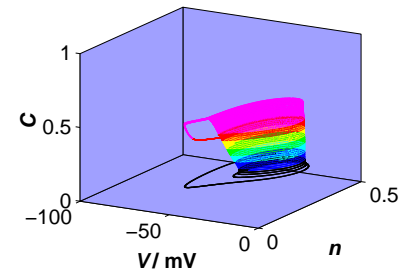

(a)

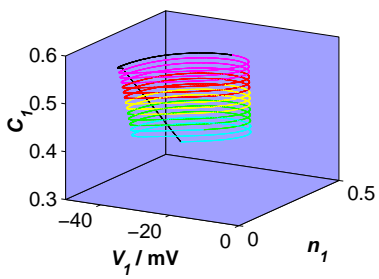

(b)

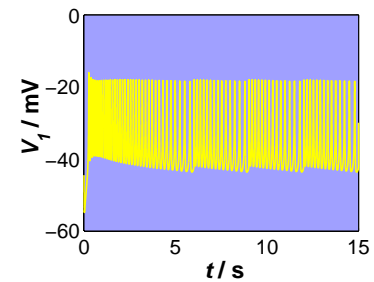

(c)

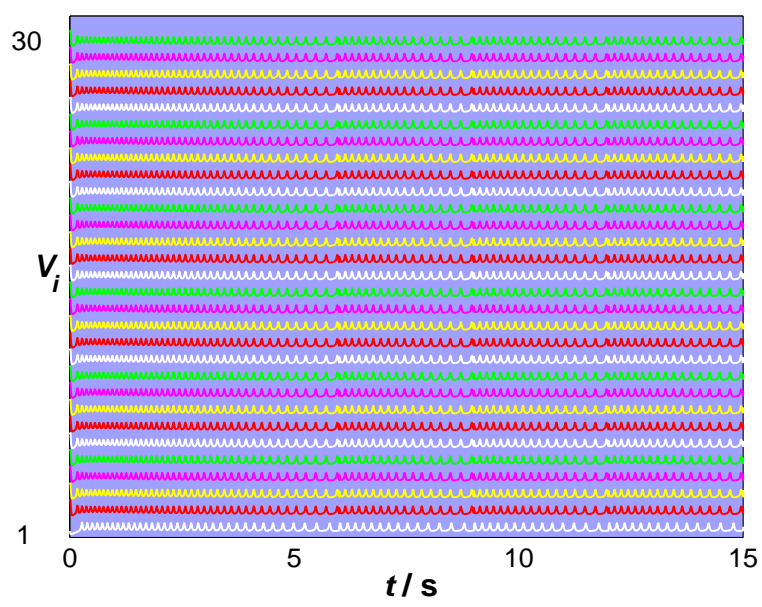

(d)

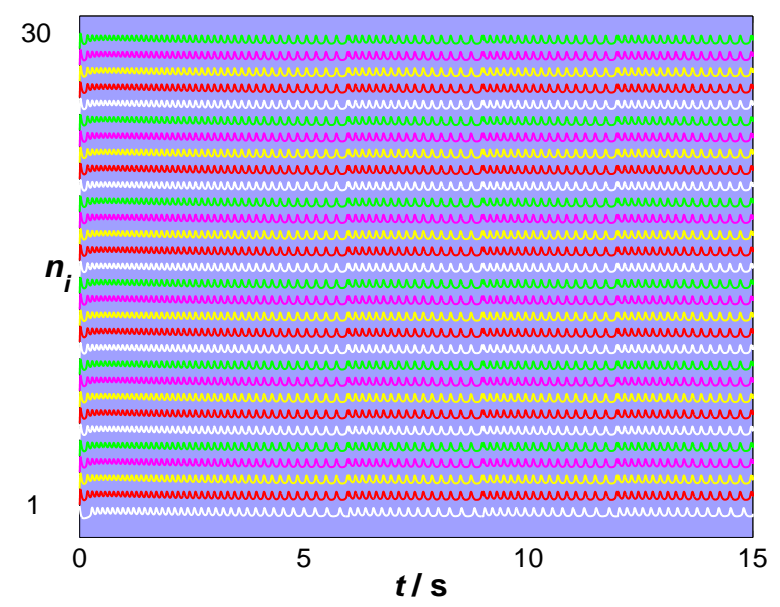

(e)

Fig. 8. Graphs generated by the parameter group No. 7 listed in Table 1. (a) The FS period trajectory of the state variables $V, C$ and $n$ of the ECM equations; initial conditions: $(V(0), C(0), n(0))=(-44.7464,0.1300,0.4661)$. (b) The trajectory of the state variables $V_{1}, C_{1}$ and $n_{1}$ of the ECM CNN converges to a 16-period limit cycle. (c) The time evolution of the state variables $V_{1}$ of the ECM CNN. (d) and (e) The graphs of the time evolution of the state variables $V_{i}^{\prime} s$ and $n_{i}^{\prime} s$ of the ECM CNN; initial conditions: $\left(V_{1}(0), C_{1}(0), n_{1}(0)\right)=(V(0), C(0), n(0))$, and $\left(V_{i}(0), C_{i}(0), n_{i}(0)\right)=(0,0,0), i=2,3, \ldots, 30$.

$$
\begin{aligned}
\Omega_{1}= & -\frac{2 a_{00}^{2}\left(a_{11}+a_{22}\right)+A_{11} A_{14}}{4\left(a_{11}+a_{22}\right)} \\
g_{1}\left(\Omega_{1}\right)= & 2\left(a_{11}+a_{22}\right) \Omega_{1}^{2}+\left[2 a_{00}^{2}\left(a_{11}+a_{22}\right)\right. \\
& \left.+A_{11} A_{14}\right] \Omega_{1}+B_{11} B_{14} \\
\Omega_{2}= & -\frac{A_{11}+A_{14}}{4\left(a_{11}+a_{22}\right)} \\
g_{2}\left(\Omega_{2}\right)= & 2\left(a_{11}+a_{22}\right) \Omega_{2}^{2} \\
& +\left(A_{11}+A_{14}\right) \Omega_{2}+B_{11}+B_{14} \\
a_{1}^{*}= & \frac{2\left(B_{11}+B_{14}\right)}{A_{11}+A_{14}} \\
a_{0}^{*}= & -b_{00}^{2}+\frac{\left(a_{00}^{2}-2 b_{00}\right)\left(B_{11}+B_{14}\right)}{A_{11}+A_{14}} \\
\Omega_{3}= & \frac{-a_{1}^{*}+\sqrt{a_{1}^{* 2}-4 a_{0}^{*}}}{2} \\
\Omega_{4}= & \frac{-a_{1}^{*}-\sqrt{a_{1}^{* 2}-4 a_{0}^{*}}}{2}
\end{aligned}
$$

$$
\begin{aligned}
g_{3}\left(\Omega_{i}\right)= & 2\left(a_{11}+a_{22}\right) \\
& +\frac{\Omega_{i}\left(A_{11}+A_{14}\right)+B_{11}+B_{14}}{\left(b_{00}-\Omega_{i}\right)^{2}+a_{00}^{2} \Omega_{i}}, \\
& i=3,4 \\
\Omega_{5}= & \frac{2 b_{00}-a_{00}}{2} \\
g_{4}\left(\Omega_{5}\right)= & 2\left(a_{11}+a_{22}\right)\left[\left(b_{00}-\Omega_{5}\right)^{2}+a_{00}^{2} \Omega_{5}\right] \\
& +B_{11}+B_{14} \\
M M= & 2 b_{00}^{2}\left[a_{22} B_{11}+a_{11} B_{14}-\left(a_{12}+a_{21}\right) B_{12}\right] \\
& +B_{11} B_{14}-B_{12}^{2} \\
J= & 2 a_{22} A_{11}+2 a_{11} A_{14}-2\left(a_{12}+a_{21}\right) A_{12} \\
& -\left(q_{12}-q_{13}\right)^{2} \\
K= & 2 a_{22} B_{11}+2 a_{22}\left(a_{00}^{2}-2 b_{00}\right) A_{11}+2 a_{11} B_{14} \\
& +2 a_{11}\left(a_{00}^{2}-2 b_{00}\right) A_{14}+A_{11} A_{14}-2\left(a_{12}\right. \\
& \left.+a_{21}\right) B_{12}-2\left(a_{12}+a_{21}\right) A_{12}\left(a_{00}^{2}-2 b_{00}\right) \\
& -A_{12}^{2}-2\left(q_{12}-q_{13}\right) B_{120}
\end{aligned}
$$




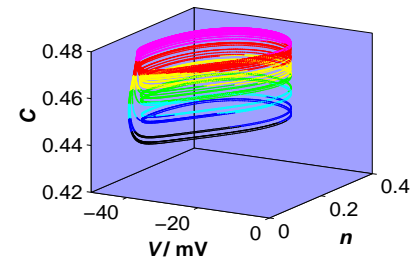

(a)

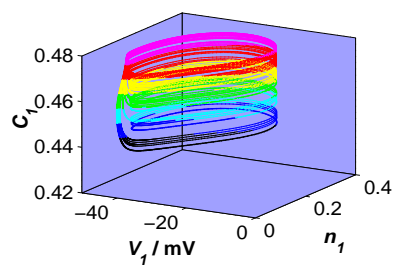

(b)

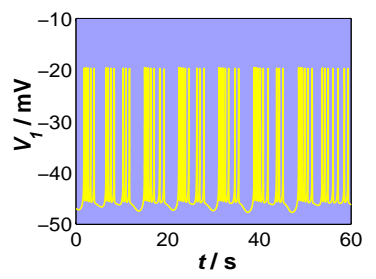

(c)

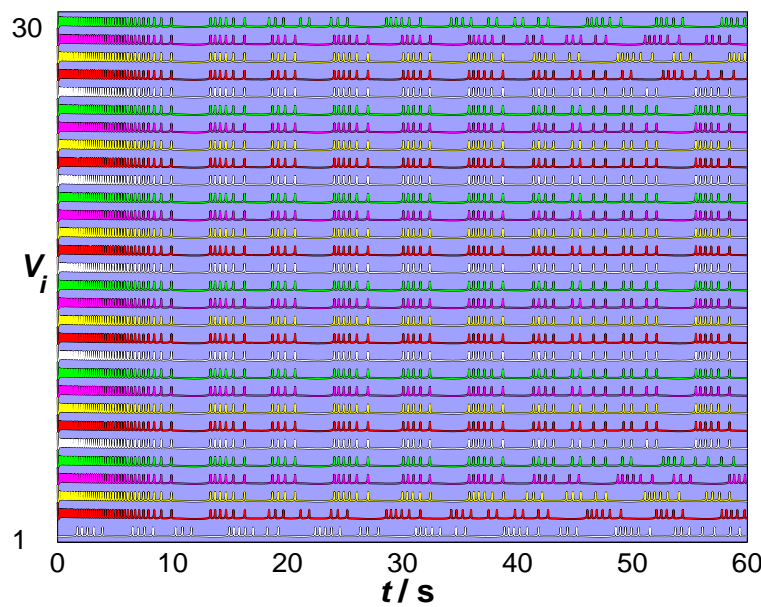

(d)

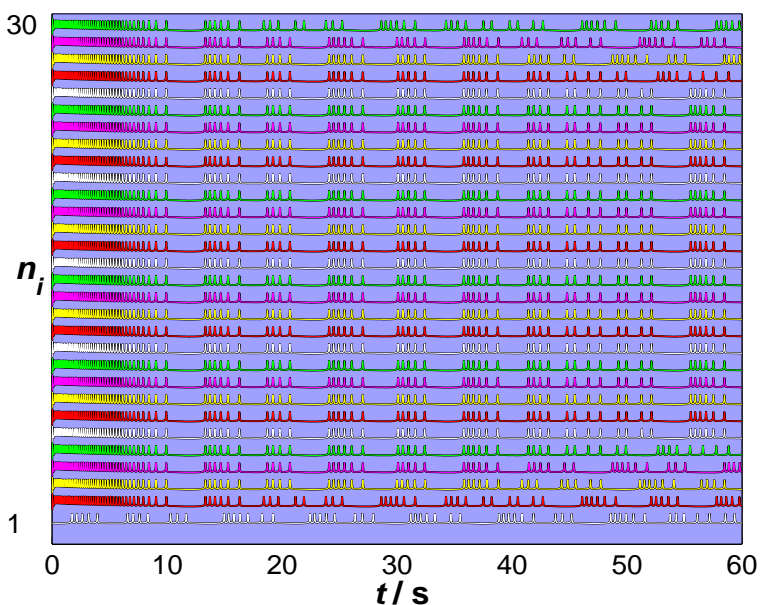

(e)

Fig. 9. Graphs generated by the parameter group No. 8 listed in Table 1. (a) The chaotic trajectory of the state variables $V$, $C$ and $n$ of the ECM equations; initial conditions: $(V(0), C(0), n(0))=(-44.7464,0.1300,0.4660)$. (b) The trajectory of the state variables $V_{1}, C_{1}$ and $n_{1}$ of the ECM CNN exhibit chaos. (c) The time evolution of the state variables $V_{1}$ of the ECM CNN. (d) and (e) The graphs of the time evolution of the state variables $V_{i}^{\prime} s$ and $n_{i}^{\prime} s$ of the ECM CNN; initial conditions: $\left(V_{1}(0), C_{1}(0), n_{1}(0)\right)=(V(0), C(0), n(0))$ and $\left(V_{i}(0), C_{i}(0), n_{i}(0)\right)=(0,0,0), i=2,3, \ldots, 30$.

$$
\begin{aligned}
L= & 2 a_{22} B_{11}\left(a_{00}^{2}-2 b_{00}\right)+2 a_{22} A_{11} b_{00}^{2} \\
& +2 a_{11} B_{14}\left(a_{00}^{2}-2 b_{00}\right)+2 a_{11} A_{14} b_{00}^{2} \\
& -2\left(a_{12}+a_{21}\right) B_{12}\left(a_{00}^{2}-2 b_{00}\right)-2\left(a_{12}\right. \\
& \left.+a_{21}\right) A_{12} b_{00}^{2}-2 A_{12} B_{12}-B_{120}^{2} \\
& +B_{11} A_{14}+A_{11} B_{14} \\
M= & 2 a_{22} B_{11} b_{00}^{2}+2 a_{11} B_{14} b_{00}^{2}-2\left(a_{12}\right. \\
& \left.+a_{21}\right) B_{12} b_{00}^{2}+B_{11} B_{14}-B_{12}^{2} \\
\Omega_{6}= & \frac{-K+\sqrt{K^{2}-3 J L}}{3 J} \\
\Omega_{7}= & \frac{-K-\sqrt{K^{2}-3 J L}}{3 J} \\
g_{5}\left(\Omega_{i}\right)= & J \Omega_{i}^{3}+K \Omega_{i}^{2}+L \Omega_{i}+M \\
& i=6,7 \\
\Omega_{8}= & -\frac{L}{2 K}
\end{aligned}
$$

$$
\begin{aligned}
g_{6}\left(\Omega_{8}\right) & =K \Omega_{8}^{2}+L \Omega_{8}+M \\
E & =4 a_{11} a_{22}-\left(a_{12}+a_{21}\right)^{2} \\
F & =2\left(a_{00}^{2}-2 b_{00}\right) E+J \\
G & =\left(a_{00}^{4}-4 a_{00}^{2} b_{00}+6 b_{00}^{2}\right) E+K \\
H & =2 b_{00}^{2}\left(a_{00}^{2}-2 b_{00}\right) E+L \\
I & =b_{00}^{4} E+M \\
w_{1} & =\frac{-1+i \sqrt{3}}{2} ; w_{2}=\frac{-1-i \sqrt{3}}{2} \\
\alpha_{0} & =\frac{3 F}{4 E}, \quad \beta_{0}=\frac{G}{2 E} \\
p & =-\frac{1}{3} \alpha_{0}^{2}+\beta_{0} \\
q & =\frac{2}{27} \alpha_{0}^{3}-\frac{\alpha_{0} \beta_{0}}{3}+\frac{H}{4 E} \\
D & =\frac{q^{2}}{4}+\frac{p^{3}}{27}
\end{aligned}
$$




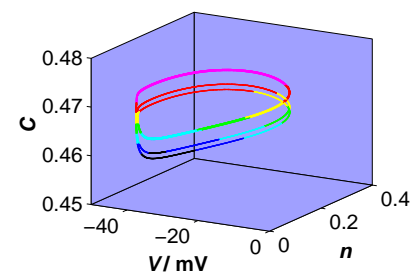

(a)

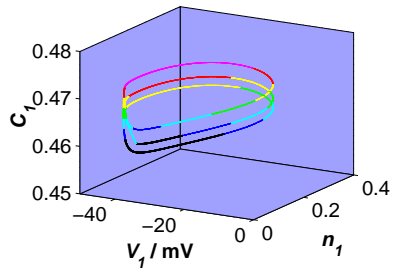

(b)

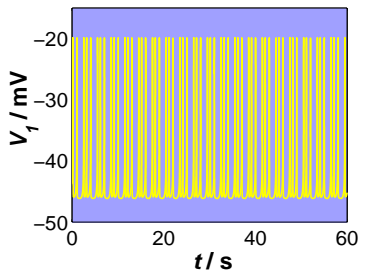

(c)

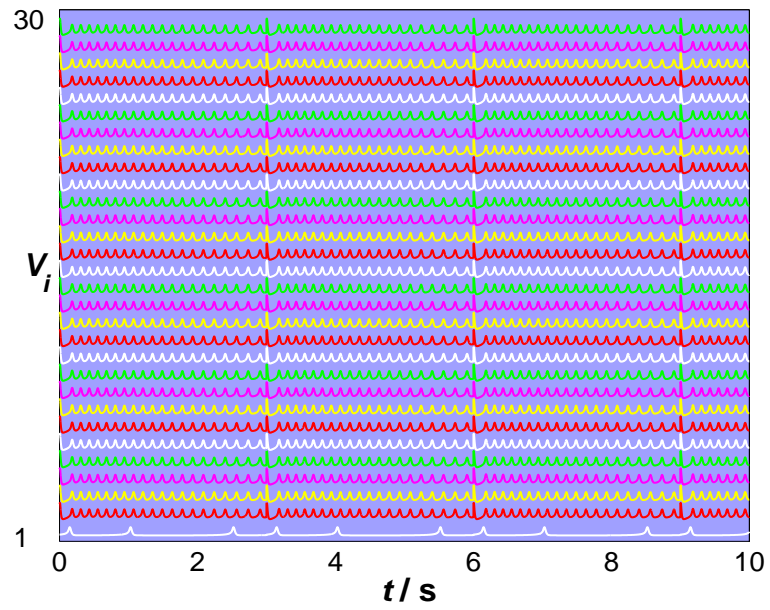

(d)

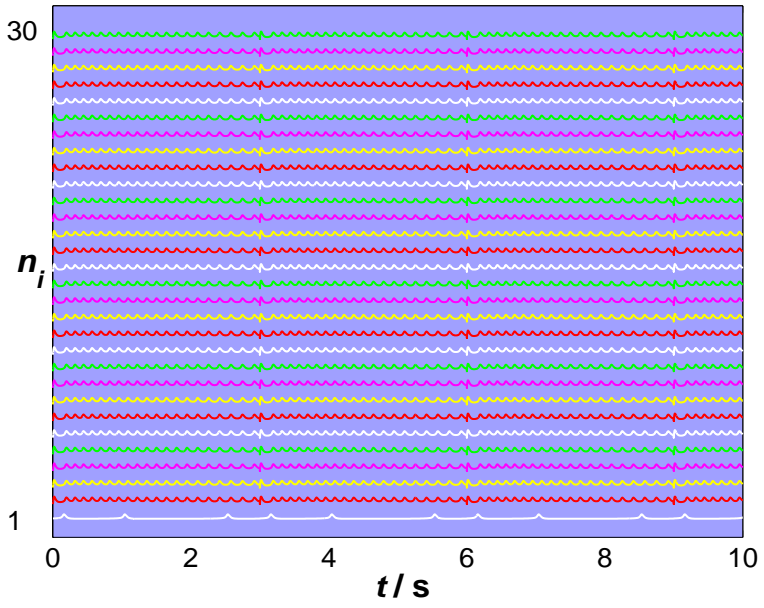

(e)

Fig. 10. Graphs generated by the parameter group No. 9 listed in Table 1. (a) The three-period trajectory of the state variables $V, C$ and $n$ of the ECM equations; initial conditions: $(V(0), C(0), n(0))=(-43.7384,0.4580,0.1374)$. (b) The trajectory of the state variables $V_{1}, C_{1}$ and $n_{1}$ of the ECM CNN converges to a three-period limit cycle. (c) The time evolution of the state variables $V_{1}$ of the ECM CNN. (d) and (e) The graphs of the time evolution of the state variables $V_{i}^{\prime} s$ and $n_{i}^{\prime} s$ of the ECM CNN; initial conditions: $\left(V_{1}(0), C_{1}(0), n_{1}(0)\right)=(V(0), C(0), n(0))$ and $\left(V_{i}(0), C_{i}(0), n_{i}(0)\right)=(0,0,0)$, $i=2,3, \ldots, 30$.

$$
\begin{gathered}
\Delta_{1}=\left\{-q / 2+\left[(q / 2)^{2}+p^{3} / 27\right]^{1 / 2}\right\}^{1 / 3} \\
+\left\{-q / 2-\left[(q / 2)^{2}+p^{3} / 27\right]^{1 / 2}\right\}^{1 / 3} \\
\Delta_{2}=w_{1}\left\{-q / 2+\left[(q / 2)^{2}+p^{3} / 27\right]^{1 / 2}\right\}^{1 / 3} \\
+w_{2}\left\{-q / 2-\left[(q / 2)^{2}+p^{3} / 27\right]^{1 / 2}\right\}^{1 / 3} \\
\Delta_{3}=w_{2}\left\{-q / 2+\left[(q / 2)^{2}+p^{3} / 27\right]^{1 / 2}\right\}^{1 / 3} \\
+w_{1}\left\{-q / 2-\left[(q / 2)^{2}+p^{3} / 27\right]^{1 / 2}\right\}^{1 / 3} \\
\Omega_{9}=\Delta_{1}-\alpha_{0} / 3 \\
\Omega_{10}=\Delta_{2}-\alpha_{0} / 3 \\
\Omega_{11}=\Delta_{3}-\alpha_{0} / 3 \\
\Omega_{12}=\frac{-G+\sqrt{G^{2}-3 F H}}{3 F} \\
\Omega_{13}=\frac{-G-\sqrt{G^{2}-3 F H}}{3 F} \\
\Omega_{14}=-\frac{H}{G}
\end{gathered}
$$

$$
\begin{aligned}
g_{7}\left(\Omega_{i}\right)= & E \Omega_{i}^{4}+F \Omega_{i}^{3}+G \Omega_{i}^{2}+H \Omega_{i}+I \\
& i=9,10, \ldots, 14
\end{aligned}
$$

Theorem 4.3. Let the following parameters be defined via formulas (36)-(45), and (48)-(94). Then, $\mathbf{Y}_{Q}(s)$ satisfies Condition 2 in the Main Theorem if, and only if, one of the following conditions holds:

(i) $a_{11}+a_{22}>0$.

(ii) $a_{11}+a_{22}=0$ and $A_{11}+A_{14}>0$.

(iii) $a_{11}+a_{22}=0, A_{11}+A_{14} \leq 0$, and $B_{11}+$ $B_{14}>0$.

(iv) $a_{11}+a_{22}<0, b_{00} \neq 0$, and $2\left(a_{11}+a_{22}\right)+$ $\left(B_{11}+B_{14}\right) / b_{00}^{2}>0$.

(v) $a_{11}+a_{22}<0, \quad b_{00}=0, a_{00} \neq 0$, and $B_{11}+B_{14}>0$.

(vi) $a_{11}+a_{22}<0, b_{00}=0, a_{00} \neq 0, B_{11}+B_{14} \leq 0$, and $\Omega_{1} \geq 0, g_{1}\left(\Omega_{1}\right)>0$.

(vii) $a_{11}+a_{22}<0, a_{00}=0, b_{00}=0$, and $\Omega_{2} \geq 0$, $g_{2}\left(\Omega_{2}\right)>0$. 


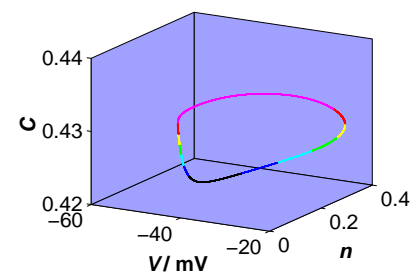

(a)

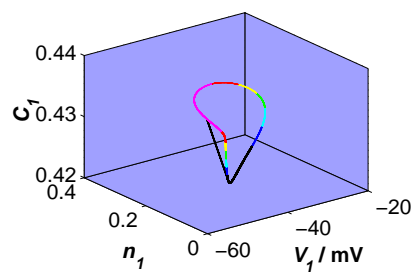

(b)

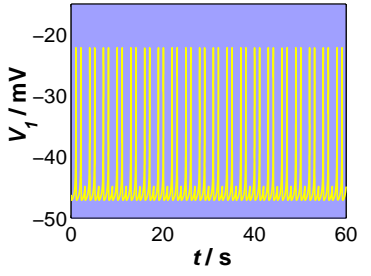

(c)

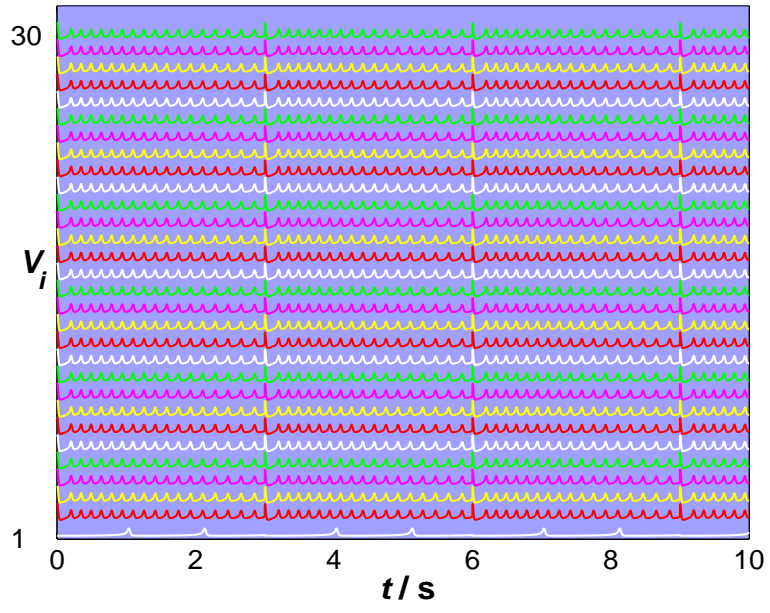

(d)

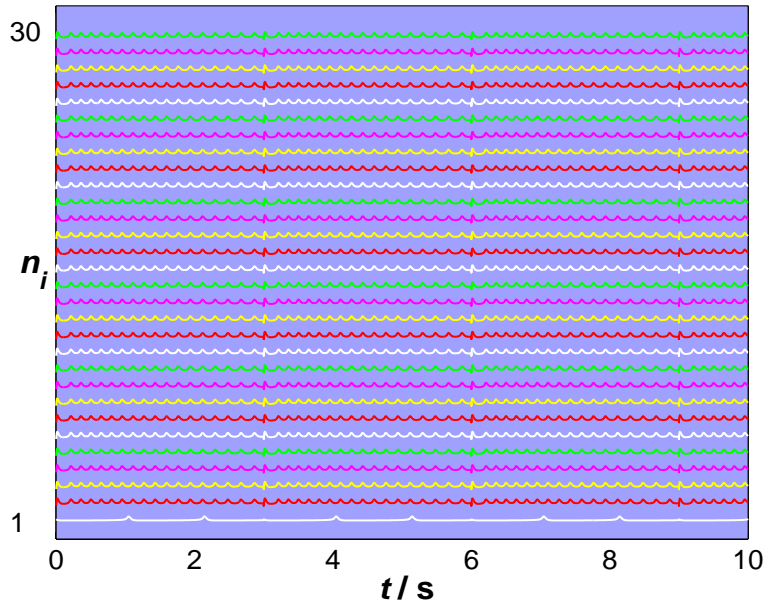

(e)

Fig. 11. Graphs generated by the parameter group No. 10 listed in Table 1. (a) The one-period trajectory of the state variables $V, C$ and $n$ of the ECM equations; initial conditions: $(V(0), C(0), n(0))=(-46.1873,0.4321,0.1816)$. (b) The trajectory of the state variables $V_{1}, C_{1}$ and $n_{1}$ of the ECM CNN converges to a two-period limit cycle. (c) The time evolution of the state variables $V_{1}$ of the ECM CNN. (d) and (e) The graphs of the time evolution of the state variables $V_{i}^{\prime} s$ and $n_{i}^{\prime} s$ of the ECM CNN; initial conditions: $\left(V_{1}(0), C_{1}(0), n_{1}(0)\right)=(V(0), C(0), n(0))$ and $\left(V_{i}(0), C_{i}(0), n_{i}(0)\right)=(0,0,0)$, $i=2,3, \ldots, 30$.

(viii) $a_{11}+a_{22}<0, a_{00} \neq 0, b_{00} \neq 0, A_{11}+A_{14} \neq 0$, and
(a) $\Omega_{3} \geq 0, g_{3}\left(\Omega_{3}\right) \geq 0$ or
(b) $\Omega_{4} \geq 0, g_{3}\left(\Omega_{4}\right) \geq 0$.

(ix) $a_{11}+a_{22}<0, a_{00} \neq 0, b_{00} \neq 0, A_{11}+A_{14}=0$, and $\Omega_{5} \geq 0, g_{4}\left(\Omega_{5}\right)>0$.

(x) $E<0$.

(xi) $E=0$ and $M M<0$.

(xii) $E=0, M M \geq 0, J \neq 0$, and
(a) $\Omega_{6} \geq 0, g_{5}\left(\Omega_{6}\right)<0$ or

(b) $\Omega_{7} \geq 0, g_{5}\left(\Omega_{7}\right)<0$.

(xiii) $E=0, M M \geq 0, J=0, K \neq 0, \Omega_{8} \geq 0$, and $g_{6}\left(\Omega_{8}\right)<0$.

(xiv) $E=0, M M \geq 0, J=K=0$, and
(a) $M<0$ or
(b) $L<0$.

(xv) $E>0$, and one of the following conditions holds: (a) $D>0, \Omega_{9} \geq 0, g_{7}\left(\Omega_{9}\right)<0$.

(b) $D<0$, and

(1) $\Omega_{9} \geq 0, g_{7}\left(\Omega_{9}\right)<0$ or

(2) $\Omega_{10} \geq 0, g_{7}\left(\Omega_{10}\right)<0$ or

(3) $\Omega_{11} \geq 0, g_{7}\left(\Omega_{11}\right)<0$.

(c) $D=0, p=q=0, \alpha_{0} \leq 0$ and $g_{7}\left(-\alpha_{0} / 3\right)<0$.

(d) $D=0, q^{2} / 4=-p^{3} / 27 \neq 0$, and

(1) $\Omega_{9} \geq 0, g_{7}\left(\Omega_{9}\right)<0$ or

(2) $\Omega_{10} \geq 0, g_{7}\left(\Omega_{10}\right)<0$.

Proof. Let

$$
\begin{aligned}
& \delta_{11}=\omega^{2} A_{11}+B_{11} ; \\
& \delta_{12}=A_{12} \omega^{2}+i \omega\left[\left(q_{13}-q_{12}\right) \omega^{2}+B_{120}\right]+B_{12} \\
& \delta_{14}=\omega^{2} A_{14}+B_{14} ; \quad \delta_{13}=\overline{\delta_{12}} .
\end{aligned}
$$

Then, 


$$
\begin{aligned}
& \left|\lambda \mathbf{I}-Y_{Q}^{H}(i \omega)\right|=\left[\begin{array}{cc}
\lambda+2 a_{11}+\frac{\delta_{11}}{\left(b_{00}-\omega^{2}\right)^{2}+\left(a_{00} \omega\right)^{2}} & \left(a_{12}+a_{21}\right)+\frac{\delta_{12}}{\left(b_{00}-\omega^{2}\right)^{2}+\left(a_{00} \omega\right)^{2}} \\
\left(a_{12}+a_{21}\right)+\frac{\delta_{13}}{\left(b_{00}-\omega^{2}\right)^{2}+\left(a_{00} \omega\right)^{2}} & \lambda+2 a_{22}+\frac{\delta_{14}}{\left(b_{00}-\omega^{2}\right)^{2}+\left(a_{00} \omega\right)^{2}}
\end{array}\right] \\
& =\left(\lambda+2 a_{11}+\frac{\omega^{2} A_{11}+B_{11}}{\left(b_{00}-\omega^{2}\right)^{2}+\left(a_{00} \omega\right)^{2}}\right)\left(\lambda+2 a_{22}+\frac{\omega^{2} A_{14}+B_{14}}{\left(b_{00}-\omega^{2}\right)^{2}+\left(a_{00} \omega\right)^{2}}\right) \\
& -\left[a_{12}+a_{21}+\frac{A_{12} \omega^{2}+B_{12}}{\left(b_{00}-\omega^{2}\right)^{2}+\left(a_{00} \omega\right)^{2}}\right]^{2}-\frac{\omega^{2}\left[\left(q_{13}-q_{12}\right) \omega^{2}+B_{120}\right]^{2}}{\left[\left(b_{00}-\omega^{2}\right)^{2}+\left(a_{00} \omega\right)^{2}\right]^{2}} \\
& \triangleq \lambda^{2}+a_{1} \lambda+a_{0} \text {. }
\end{aligned}
$$

Consequently, if $a_{1}>0$ or $a_{0}<0$, then $\mathbf{Y}_{Q}(s)$ satisfies Condition 2 in Main Theorem.

Case 1. Conditions for $a_{1}>0$.

$a_{1}=2\left(a_{11}+a_{22}\right)+\frac{\left(A_{11}+A_{14}\right) \omega^{2}+B_{11}+B_{14}}{\left(b_{00}-\omega^{2}\right)^{2}+a_{00}^{2} \omega^{2}}$.

(I) If $a_{11}+a_{22}>0$, then $a_{1}>0$, when $\omega$ is large enough [Theorem 4.3(i)].

(II) If $a_{11}+a_{22}=0$, then $a_{1}>0 \Leftrightarrow\left(A_{11}+A_{14}\right) \Omega+$ $B_{11}+B_{14}>0$. Therefore if

(A) $A_{11}+A_{14}>0, a_{11}+a_{22}=0$, then $a_{1}>0$ as $\omega$ is large enough [Theorem 4.3(ii)];

(B) $A_{11}+A_{14} \leq 0, a_{11}+a_{22}=0$ and $B_{11}+B_{14}>0$, then $a_{1}>0$ as $\omega=0$ [Theorem 4.3(iii)].

(III) If $a_{11}+a_{22}<0$,

(A) $a_{11}+a_{22}<0, b_{00} \neq 0$ and $2\left(a_{11}+a_{22}\right)+$ $\left[\left(B_{11}+B_{14}\right) / b_{00}^{2}\right]>0$ then $a_{1}>0$, for $\omega=0$ [Theorem 4.3(iv)].

(B) If $a_{11}+a_{22}<0, b_{00}=0, a_{00} \neq 0$, solve

$$
\begin{aligned}
a_{1}(\Omega)= & g_{1}(\Omega)=2\left(a_{11}+a_{22}\right) \\
& +\frac{\left(A_{11}+A_{14}\right) \Omega+B_{11}+B_{14}}{\left(b_{00}-\Omega\right)^{2}+a_{00}^{2} \Omega}>0 .
\end{aligned}
$$

We can first solve

$$
\begin{aligned}
& {\left[2\left(a_{11}+a_{22}\right)\left(\Omega^{2}+a_{00}^{2} \Omega\right)\right.} \\
& \left.\quad+\left(A_{11}+A_{14}\right) \Omega+B_{11}+B_{14}\right]^{\prime}=0, \\
& 4 \Omega\left(a_{11}+a_{22}\right)+\left[2 a_{00}^{2}\left(a_{11}+a_{22}\right)+A_{11}+A_{14}\right]=0 .
\end{aligned}
$$

We obtain

$$
\Omega_{1}=\frac{-\left[2 a_{00}^{2}\left(a_{11}+a_{22}\right)+A_{11}+A_{14}\right]}{4\left(a_{11}+a_{22}\right)} .
$$

Therefore if $\Omega_{1} \geq 0$ and $g_{1}\left(\Omega_{1}\right)>0$, then $a_{1}>0$. It follows that

(B1) $a_{11}+a_{22}<0, \quad b_{00}=0, a_{00} \neq 0$, $B_{11}+B_{14}>0$, then $a_{1}>0$ [Theorem $4.3(\mathrm{v})]$.

(B2) $a_{11}+a_{22}<0, \quad b_{00}=0, a_{00} \neq 0$, $B_{11}+B_{14} \leq 0, \Omega_{1} \geq 0$ and $g_{1}\left(\Omega_{1}\right)>0$, then $a_{1}>0$ [Theorem 4.3(vi)].

(C) $a_{11}+a_{22}<0, b_{00}=a_{00}=0$, then $a_{1}>0$ becomes

$$
\begin{aligned}
a_{1}(\Omega)= & 2\left(a_{11}+a_{22}\right) \\
& +\frac{\left(A_{11}+A_{14}\right) \Omega+B_{11}+B_{14}}{\Omega^{2}} \\
& >0 . \\
\text { Let } \quad & \\
g_{2}(\Omega)= & 2 \Omega^{2}\left(a_{11}+a_{22}\right)+\left(A_{11}+A_{14}\right) \Omega \\
& +B_{11}+B_{14} .
\end{aligned}
$$

Solving

$$
\begin{aligned}
g_{2}^{\prime}(\Omega) & =0 \Rightarrow 4 \Omega\left(a_{11}+a_{22}\right)+A_{11}+A_{14} \\
& =0,
\end{aligned}
$$

we obtain

$$
\Omega_{2}=\frac{-A_{11}-A_{14}}{4\left(a_{11}+a_{22}\right)} \geq 0 .
$$

It follows that if $a_{11}+a_{22}<0, a_{00}=$ $b_{00}=0, \Omega_{2} \geq 0$ and $g_{2}\left(\Omega_{2}\right)>0$, then $a_{1}>0$ [Theorem 4.3(vii)].

(D) $a_{11}+a_{22}<0, a_{00} \neq 0, b_{00} \neq 0$. Let

$$
\begin{aligned}
a_{1}= & g_{3}(\Omega)=g_{3}\left(\omega^{2}\right) \\
= & 2\left(a_{11}+a_{22}\right) \\
& +\frac{\left(A_{11}+A_{14}\right) \Omega+B_{11}+B_{14}}{\left(b_{00}-\Omega\right)^{2}+a_{00}^{2} \Omega} .
\end{aligned}
$$


Solving $g_{3}^{\prime}(\Omega)=0$, we obtain

$$
\begin{aligned}
\left(A_{11}\right. & \left.+A_{14}\right)\left[\left(b_{00}-\Omega\right)^{2}+a^{2} \Omega\right] \\
& -\left[\left(A_{11}+A_{14}\right) \Omega+B_{11}\right. \\
& \left.+B_{14}\right]\left[-2\left(b_{00}-\Omega\right)+a^{2}\right]=0 .
\end{aligned}
$$

(D1) If $A_{11}+A_{14} \neq 0$, then

$$
\begin{aligned}
\Omega^{2} & +\frac{2\left(B_{11}+B_{14}\right)}{A_{11}+A_{14}} \Omega-b_{00}^{2} \\
+ & \frac{\left(a_{00}^{2}-2 b_{00}\right)\left(B_{11}+B_{14}\right)}{A_{11}+A_{14}}=0 .
\end{aligned}
$$

From the above equation, we get

$$
\Omega_{3,4}=\frac{-a_{1}^{*} \pm \sqrt{a_{1}^{* 2}-4 a_{0}^{*}}}{2} .
$$

We conclude that if $a_{11}+a_{22}<0$, $a_{00} \neq 0, b_{00} \neq 0, A_{11}+A_{14} \neq 0$, and

(a) $\Omega_{3} \geq 0$ and $g_{3}\left(\Omega_{3}\right)>0$, or

(b) $\Omega_{4} \geq 0$ and $g_{4}\left(\Omega_{4}\right)>0$, then $a_{1}>0$ [Theorem 4.3(viii)].

(D2) If $A_{11}+A_{14}=0$, then $a_{1}=2\left(a_{11}+\right.$ $\left.a_{22}\right)+\left(B_{11}+B_{14}\right) /\left[\left(b_{00}-\Omega\right)^{2}+a_{00}^{2} \Omega\right]$.

Let

$$
\begin{aligned}
g_{4}(\Omega)= & 2\left(a_{11}+a_{22}\right)\left[\left(b_{00}-\Omega\right)^{2}+a_{00}^{2} \Omega\right] \\
& +B_{11}+B_{14}>0 .
\end{aligned}
$$

Solving

$$
g_{4}(\Omega)=0,
$$

that is $-2\left(b_{00}-\Omega\right)+a_{00}^{2}=0$, we obtain

$$
\Omega_{5}=\frac{2 b_{00}-a_{00}^{2}}{2} .
$$

Therefore, if $a_{11}+a_{22}<0, a_{00} \neq 0, b_{00} \neq 0$, $A_{11}+A_{14}=0, \Omega_{5} \geq 0$ and $g_{4}\left(\Omega_{5}\right)>0$, then $a_{1}>0$ [Theorem 4.3(ix)].

Case 2. Conditions for $a_{0}<0$.

$$
\begin{aligned}
a_{0}= & {\left[2 a_{11}+\frac{\omega^{2} A_{11}+B_{11}}{\left(b_{00}-\omega^{2}\right)^{2}+a_{00}^{2} \omega^{2}}\right] } \\
& \times\left[2 a_{22}+\frac{\omega^{2} A_{14}+B_{14}}{\left(b_{00}-\omega^{2}\right)^{2}+a_{00}^{2} \omega^{2}}\right] \\
& -\left[a_{11}+a_{22}+\frac{\omega^{2} A_{12}+B_{12}}{\left(b_{00}-\omega^{2}\right)^{2}+a_{00}^{2} \omega^{2}}\right]^{2} \\
& -\left\{\frac{\omega\left[\left(q_{13}-q_{12}\right) \omega^{2}+B_{120}\right]}{\left(b_{00}-\omega^{2}\right)^{2}+a_{00}^{2} \omega^{2}}\right\}^{2} .
\end{aligned}
$$

(I) If $4 a_{11} a_{22}-\left(a_{12}+a_{21}\right)^{2}<0$, then $a_{0}<0$ as $\omega$ is large enough [Theorem $4.3(\mathrm{x})]$.

(II) (A) If $E=0$ and $b_{00} \neq 0$, let $g(\omega)=a_{0}(\omega)$. Then

$$
\begin{aligned}
& g(\omega) \\
& =4 a_{11} a_{22} \\
& +\frac{2 a_{22}\left(\omega^{2} A_{11}+B_{11}\right)+2 a_{11}\left(\omega^{2} A_{14}+B_{14}\right)}{\left(b_{00}-\omega^{2}\right)^{2}+\left(a_{00} \omega\right)^{2}} \\
& +\frac{\left(\omega^{2} A_{11}+B_{11}\right)\left(\omega^{2} A_{14}+B_{14}\right)}{\left[\left(b_{00}-\omega^{2}\right)^{2}+\left(a_{00} \omega\right)^{2}\right]^{2}} \\
& -\frac{2\left(a_{12}+a_{21}\right)\left(A_{12} \omega^{2}+B_{12}\right)}{\left(b_{00}-\omega^{2}\right)^{2}+\left(a_{00} \omega\right)^{2}} \\
& -\frac{\left(\omega^{2} A_{12}+B_{12}\right)^{2}}{\left[\left(b_{00}-\omega^{2}\right)^{2}+\left(a_{00} \omega\right)^{2}\right]^{2}} \\
& -\frac{\omega^{2}\left[\left(q_{13}-q_{12}\right) \omega^{2}+B_{120}\right]^{2}}{\left[\left(b_{00}-\omega^{2}\right)^{2}+\left(a_{00} \omega\right)^{2}\right]^{2}} \\
& -\left(a_{12}+a_{21}\right)^{2} .
\end{aligned}
$$

Let $\Omega=\omega^{2}$. If

$$
\begin{aligned}
M M & =2 b_{00}^{2}\left[a_{22} B_{11}+a_{11} B_{14}-\left(a_{12}+a_{21}\right) B_{12}\right] \\
& =b_{11} B_{14}-B_{12}^{2}<0,
\end{aligned}
$$

then $a_{0}<0, g(\Omega)<0$ as $\omega=0$ [Theorem 4.3(xi)].

(B) $4 a_{11} a_{22}-\left(a_{12}+a_{21}\right)^{2}=0$, and $M M \geq 0$. Let

$$
g_{5}(\Omega)=J \Omega^{3}+K \Omega^{2}+L \Omega+M .
$$

Solving

$$
g_{5}^{\prime}(\Omega)=0
$$

we obtain

$$
3 J \Omega^{2}+2 K \Omega+L=0 .
$$

(B1) $J \neq 0$, solving the above equation, we obtain

$$
\Omega_{6,7}=\frac{-K \pm \sqrt{K^{2}-3 J L}}{3 J} .
$$

It follows that if $4 a_{11} a_{22}-\left(a_{12}+a_{21}\right)^{2}=$ $0, M M \geq 0, J \neq 0$, and 
(a) $\Omega_{6} \geq 0$ and $g_{5}\left(\Omega_{6}\right)<0$, or

(b) $\Omega_{7} \geq 0$ and $g_{5}\left(\Omega_{7}\right)<0$, then $a_{0}<0$ [Theorem 4.3(xii)].

(B2) $J \neq 0$ and $K \neq 0$. Let

$$
g_{6}(\Omega)=K \Omega^{2}+L \Omega+M .
$$

Solving

$$
g_{6}^{\prime}(\Omega)=2 K \Omega+L=0,
$$

we obtain

$$
\Omega_{8}=-\frac{L}{2 K}
$$

It follows that if $4 a_{11} a_{22}-\left(a_{12}+a_{21}\right)^{2}=$ 0 , and $M M \geq 0, J=0, k \neq 0, \Omega_{8} \geq 0$ and $g_{6}\left(\Omega_{8}\right)<0$, then $a_{0}<0$ [Theorem 4.3 (xiii)].

(C) If $J=K=0$, then $g_{6}(\Omega)=L \Omega+M$. Therefore, if $4 a_{11} a_{22}-\left(a_{12}+a_{21}\right)^{2}=0$, and $M M \geq 0, J=0, K=0$ and
(a) $M<0$ or
(b) $L<0$,

then $a_{0}<0$ [Theorem 4.3(xiv)].

(III) $4 a_{11} a_{22}-\left(a_{12}+a_{21}\right)^{2}>0$,

$$
\begin{aligned}
a_{0} & =g_{7}(\Omega) \\
& =E \Omega^{4}+F \Omega^{3}+G \Omega^{2}+H \Omega+I .
\end{aligned}
$$

If $E \neq 0$, denote

$$
\begin{aligned}
p & =-3 F^{2} / 16 E^{2}+G / 2 E \\
q & =F^{3} / 32 E^{3}-F G / 8 E^{2}+H / 4 E \\
D & =q^{2} / 4+p^{3} / 27 .
\end{aligned}
$$

Then, from the formulas for the roots of a cub polynomial, we can conclude that $a_{0}<0$ [Theorem 4.3(xv)] if, and only if, one of the following conditions holds:

(a) $D>0, \Omega_{9}>0, g_{7}\left(\Omega_{9}\right)<0$,

(b) $D<0$ and

(1) $\Omega_{9} \geq 0$ and $g_{7}\left(\Omega_{9}\right)<0$ or

(2) $\Omega_{10} \geq 0$ and $g_{7}\left(\Omega_{10}\right)<0$ or

(3) $\Omega_{11} \geq 0$ and $g_{7}\left(\Omega_{11}\right)<0$.

(c) $D=0, p=0, q=0, \alpha_{0} \leq 0$ and

$$
\begin{aligned}
g_{7}(\Omega)= & g_{7}\left(-\alpha_{0} / 3\right) \\
= & E\left(\alpha_{0} / 3\right)^{4}-F\left(\alpha_{0} / 3\right)^{3}+G\left(\alpha_{0} / 3\right)^{2} \\
& -H\left(\alpha_{0} / 3\right)+I<0
\end{aligned}
$$

(d) $D=0, q^{2} / 4=-p^{3} / 27 \neq 0$

(1) $\Omega_{9} \geq 0$ and $g_{7}\left(\Omega_{9}\right)<0$ or

(2) $\Omega_{10} \geq 0$ and $g_{7}\left(\Omega_{10}\right)<0$.

In summary, we have completed the proof.

Theorem 4.4. For $i=1,2,3,4$, let $q_{1 i}, q_{1 i 0}, a_{00}$ and $b_{00}$ be defined by formulas (36)-(45). Then, $\mathbf{Y}_{Q}(s)$ has a multiple pole on the imaginary axis if, and only if, $a_{00}=b_{00}=0$, and

$$
\begin{aligned}
& \max \left\{\left|q_{11}\right|+\left|q_{110}\right|,\left|q_{12}\right|+\left|q_{120}\right|,\left|q_{13}\right|+\left|q_{130}\right|,\right. \\
& \left.\left|q_{14}\right|+\left|q_{140}\right|\right\} \neq 0
\end{aligned}
$$

Proof. $\quad \mathbf{Y}_{Q}(s)$ can only have a double pole 0 on the imaginary axis. It is true if, and only if, the above equation holds.

In summary, Theorems 4.1-4.4 provide the analytic criteria for two-port CNN with four state variables.

\section{2. $S C C C N N$ and bifurcation diagrams}

The two-port SCC with four state variables and $15 \times 15$ arrays is similar to the one-port SCC given in [Min et al., 2000], which might be the most friendly model that either has very complex dynamical behaviors or can be studied easily via the local activity theory. The 4D SCC equations are represented as follows.

$$
\begin{aligned}
\frac{d x_{1}}{d t}=\alpha\left[x_{2}-x_{1}-b x_{1}\right. & \left.-\frac{(a-b)}{\pi} \arctan \left(5 x_{1}\right)\right] \\
\frac{d x_{2}}{d t}= & x_{1}-x_{2}+x_{3} \\
\frac{d x_{3}}{d t}= & -\beta x_{2} \\
\frac{d x_{4}}{d t}= & \alpha\left[x_{1}-x_{4}-2 b x_{1}\right. \\
& \left.-\frac{2(a-b)}{\pi} \arctan \left(5 x_{1}\right)\right],
\end{aligned}
$$

where $\alpha, \beta, a$ and $b$ are parameters. 

by

The corresponding two-port SCC CNN is given

$$
\begin{aligned}
\dot{x}_{1 i, j}= & \alpha\left[x_{2 i, j}-x_{1 i, j}-b x_{1 i, j}-\frac{(a-b)}{\pi}\right. \\
& \left.\times \arctan \left(5 x_{1 i, j}\right)\right]+D_{1}\left[x_{1 i+1, j}+x_{1 i-1, j}\right. \\
& \left.+x_{1 i, j+1}+x_{1 i, j-1}-4 x_{1 i, j}\right] \\
\dot{x}_{2 i, j}= & x_{1 i, j}-x_{2 i, j}+x_{3 i, j}+D_{2}\left[x_{2 i+1, j}+x_{2 i-1, j}\right. \\
& \left.+x_{2 i, j+1}+x_{2 i, j-1}-4 x_{2 i, j}\right] \\
\dot{x}_{3 i, j}= & -\beta x_{2 i, j} \\
\dot{x}_{4 i, j}= & \alpha\left[x_{1 i, j}-x_{4 i, j}-2 b x_{1 i, j}\right. \\
& \left.-\frac{2(a-b)}{\pi} \arctan \left(5 x_{1 i, j}\right)\right] \\
& i, j=1,2, \ldots, 15 .
\end{aligned}
$$

Now, let us revise Eqs. (100)-(103) into four equations of $225 \times 1$ vector differential equations, via choosing a row-wise order scheme:

$$
\begin{aligned}
x_{1 i, j} \mid \longrightarrow & x_{1}^{(i-1) N+j} \\
x_{2 i, j} \mid \longrightarrow & x_{2}^{(i-1) N+j} \\
x_{3 i, j} \mid \longrightarrow & x_{3}^{(i-1) N+j} \\
x_{4 i, j} \mid \longrightarrow & x_{4}^{(i-1) N+j} \\
& i, j=1,2, \ldots, 15 . \\
\mathbf{X}_{1}= & {\left[x_{1}^{1}, x_{1}^{2}, \ldots, x_{1}^{225}\right] } \\
\mathbf{X}_{2}= & {\left[x_{2}^{1}, x_{2}^{2}, \ldots, x_{2}^{225}\right] } \\
\mathbf{X}_{3}= & {\left[x_{3}^{1}, x_{3}^{2}, \ldots, x_{3}^{225}\right] } \\
\mathbf{X}_{4}= & {\left[x_{4}^{1}, x_{4}^{2}, \ldots, x_{4}^{225}\right] . }
\end{aligned}
$$

Consequently, in a component form, Eqs. (100)-(103) become

$$
\begin{aligned}
& \dot{\mathbf{X}}_{\mathbf{1}}=f_{1}\left(\mathbf{X}_{\mathbf{1}}, \mathbf{X}_{\mathbf{2}}, \mathbf{X}_{\mathbf{3}}, \mathbf{X}_{\mathbf{4}}\right)+D_{1} \nabla^{2} \mathbf{X}_{\mathbf{1}} \\
& \dot{\mathbf{X}}_{\mathbf{2}}=f_{2}\left(\mathbf{X}_{\mathbf{1}}, \mathbf{X}_{\mathbf{2}}, \mathbf{X}_{\mathbf{3}}, \mathbf{X}_{\mathbf{4}}\right)+D_{2} \nabla^{2} \mathbf{X}_{\mathbf{2}} \\
& \dot{\mathbf{X}}_{\mathbf{3}}=f_{3}\left(\mathbf{X}_{\mathbf{1}}, \mathbf{X}_{\mathbf{2}}, \mathbf{X}_{\mathbf{3}}, \mathbf{X}_{\mathbf{4}}\right) \\
& \dot{\mathbf{X}}_{\mathbf{4}}=f_{4}\left(\mathbf{X}_{1}, \mathbf{X}_{\mathbf{2}}, \mathbf{X}_{\mathbf{3}}, \mathbf{X}_{\mathbf{4}}\right)
\end{aligned}
$$

where

$$
\begin{aligned}
& f_{1}\left(\mathbf{X}_{\mathbf{1}}, \mathbf{X}_{\mathbf{2}}, \mathbf{X}_{\mathbf{3}}, \mathbf{X}_{\mathbf{4}}\right) \\
& \quad=\alpha\left[\mathbf{X}_{\mathbf{2}}-\mathbf{X}_{\mathbf{1}}-b \mathbf{X}_{\mathbf{1}}-\frac{(a-b)}{\pi} \arctan \left(5 \mathbf{X}_{\mathbf{1}}\right)\right]
\end{aligned}
$$

$$
\begin{aligned}
& f_{2}\left(\mathbf{X}_{\mathbf{1}}, \mathbf{X}_{\mathbf{2}}, \mathbf{X}_{\mathbf{3}}, \mathbf{X}_{\mathbf{4}}\right)=\mathbf{X}_{\mathbf{1}}-\mathbf{X}_{\mathbf{2}}+\mathbf{X}_{\mathbf{3}}(109) \\
& f_{3}\left(\mathbf{X}_{1}, \mathbf{X}_{\mathbf{2}}, \mathbf{X}_{\mathbf{3}}, \mathbf{X}_{\mathbf{4}}\right)=-\beta \mathbf{X}_{\mathbf{2}} \\
& f_{4}\left(\mathbf{X}_{\mathbf{1}}, \mathbf{X}_{\mathbf{2}}, \mathbf{X}_{\mathbf{3}}, \mathbf{X}_{\mathbf{4}}\right) \\
& =\alpha\left[\mathbf{X}_{\mathbf{1}}-\mathbf{X}_{\mathbf{4}}-2 b \mathbf{X}_{\mathbf{1}}-2 \frac{(a-b)}{\pi} \arctan \left(5 \mathbf{X}_{\mathbf{1}}\right)\right]
\end{aligned}
$$

and $\nabla^{2}$ corresponds to a $225 \times 225$ matrix.

The cell equilibrium points $Q_{i}^{\prime} s$ of Eqs. (104)(107) for the restricted local activity domain [Dogaru \& Chua, 1998a] can be determined numerically via

$$
\begin{aligned}
& f_{1}\left(\mathbf{X}_{\mathbf{1}}, \mathbf{X}_{\mathbf{2}}, \mathbf{X}_{\mathbf{3}}, \mathbf{X}_{\mathbf{4}}\right)=0 \\
& f_{2}\left(\mathbf{X}_{\mathbf{1}}, \mathbf{X}_{\mathbf{2}}, \mathbf{X}_{\mathbf{3}}, \mathbf{X}_{\mathbf{4}}\right)=0 \\
& f_{3}\left(\mathbf{X}_{\mathbf{1}}, \mathbf{X}_{\mathbf{2}}, \mathbf{X}_{\mathbf{3}}, \mathbf{X}_{\mathbf{4}}\right)=0 \\
& f_{4}\left(\mathbf{X}_{\mathbf{1}}, \mathbf{X}_{\mathbf{2}}, \mathbf{X}_{\mathbf{3}}, \mathbf{X}_{\mathbf{4}}\right)=0
\end{aligned}
$$

From Eqs. (108)-(115), it follows that for any parameter group $\{\alpha, \beta, a, b\}$, there exists at least one cell equilibrium point:

$$
Q_{1}=(0,0,0,0) .
$$

On the other hand, the other cell equilibrium point $Q_{i}=\left(x_{1}, x_{2}, x_{3}, x_{4}\right)$ must satisfy the following equations:

$$
\begin{aligned}
\mathbf{X}_{2} & =0 \\
\mathbf{X}_{3} & =-\mathbf{X}_{1} \\
0 & =(1+b) \mathbf{X}_{1}+\frac{(a-b)}{\pi} \arctan \left(5 \mathbf{X}_{1}\right) \\
0 & =\mathbf{X}_{1}-\mathbf{X}_{4}-2 b \mathbf{X}_{1}-2 \frac{(a-b)}{\pi} \arctan \left(5 \mathbf{X}_{1}\right) .
\end{aligned}
$$

Mathematical analysis shows that Eqs. (116)-(119) have just two nonzero equilibrium points and are determined by the following:

Constraint condition [Min et al., 2000b]. If $Q_{2}$ is a nonzero equilibrium point, then $Q_{3}=-Q_{2}$ is also an equilibrium point, and the parameters $a$ and $b$ have to satisfy the following inequality:

$$
(b+1)<5(b-a) / \pi .
$$


Using the constraint condition (120), the admissible parameter domain for the nonzero equilibrium points $Q_{2}$ and $Q_{3}$ can be calculated via computer programming. Consequently, the cell coefficients $a_{m, n}\left(Q_{i}\right)^{\prime} s$ are well defined via the corresponding Jacobian matrix:

$$
\begin{aligned}
\mathbf{A}_{i} \triangleq & {\left[\begin{array}{llll}
a_{11}\left(Q_{i}\right) & a_{12}\left(Q_{i}\right) & a_{13}\left(Q_{i}\right) & a_{14}\left(Q_{i}\right) \\
a_{21}\left(Q_{i}\right) & a_{22}\left(Q_{i}\right) & a_{23}\left(Q_{i}\right) & a_{24}\left(Q_{i}\right) \\
a_{31}\left(Q_{i}\right) & a_{32}\left(Q_{i}\right) & a_{33}\left(Q_{i}\right) & a_{34}\left(Q_{i}\right) \\
a_{41}\left(Q_{i}\right) & a_{42}\left(Q_{i}\right) & a_{43}\left(Q_{i}\right) & a_{44}\left(Q_{i}\right)
\end{array}\right] } \\
& =\left[\begin{array}{cccc}
a_{11}\left(Q_{i}\right) & a_{12}\left(Q_{i}\right) & 0 & 0 \\
a_{21}\left(Q_{i}\right) & a_{22}\left(Q_{i}\right) & a_{23}\left(Q_{i}\right) & 0 \\
0 & a_{32}\left(Q_{i}\right) & 0 & 0 \\
a_{41}\left(Q_{i}\right) & 0 & 0 & a_{44}\left(Q_{i}\right)
\end{array}\right]
\end{aligned}
$$

where

$$
\left\{\begin{array}{l}
a_{11}=\alpha\left\{-1-\left[b+\frac{5(a-b)}{\pi\left(1+\left(5 x_{1}\right)^{2}\right)}\right]\right\}, \\
a_{12}=\alpha, a_{21}=1, a_{22}=-1, \\
a_{23}=1, a_{32}=-\beta, a_{44}=-\alpha, \\
a_{41}=\alpha\left\{-1-2\left[b+\frac{5(a-b)}{\pi\left(1+\left(5 x_{1}\right)^{2}\right)}\right]\right\} .
\end{array}\right.
$$

Using Theorems 4.1-4.4, constrain condition (120) and formulas (35)-(45), (121) and (122), the locally active domains, locally passive domains and edges of chaos with respect to the equilibrium points $Q_{1}$ and $Q_{2}$ (resp. $Q_{3}$ ) with different cell parameters, are shown in Figs. 12(a) and 12(b), respectively. From these graphs, it can be concluded that:

- For all the selected parameter groups, the corresponding bifurcation diagrams do not have locally passive domains.

- Although the analytical criteria for the four variable CNNs with one port (see Theorems 2.1-2.4, and Figs. 5(a) and 5(b) in [Min et al., 2000b]) and two ports (see Theorems 4.1-4.4) are formally different, the two bifurcation graphs, Figs. 12(a) and 12(b), are the same as those shown in Figs. 5(a) and $5(\mathrm{~b})$, respectively.

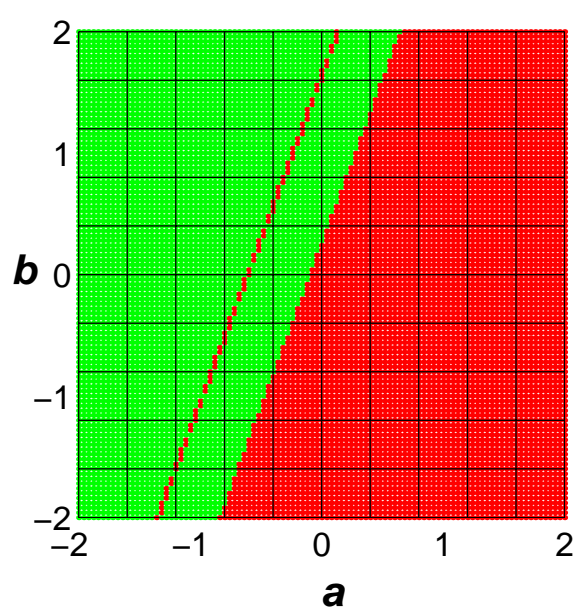

(a)

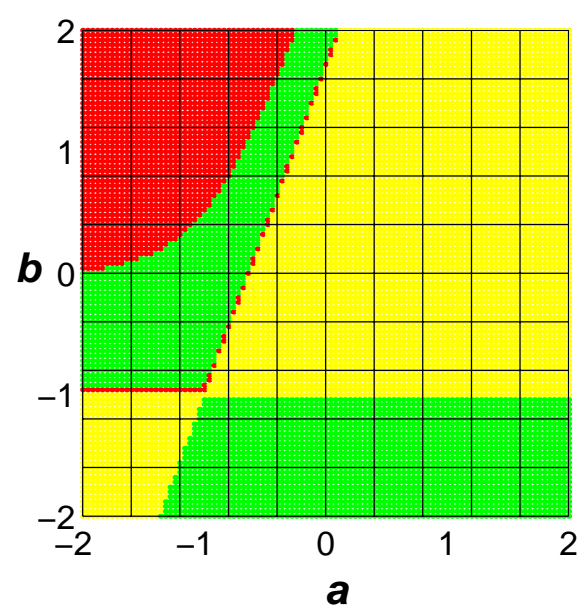

(b)

Fig. 12. Bifurcation diagrams of the SCC two-diffusion CNNs with parameters $\alpha=10$ and $\beta=15$. The domains are coded as follows: edge of chaos (locally active and stable) domain (red), locally active and unstable domain (green). In the domain coded with yellow, no nonzero equilibrium point exists. The edge of chaos with respect to the equilibrium point: (a) $Q_{1}(=0)$ at cross-section $a \in[-2,2]$ and $b \in[-2,2]$ and (b) $Q_{2}\left(\right.$ resp. $\left.Q_{3}\right)$ at cross-section $a \in[-2,2]$ and $b \in[-2,2]$.

- The edges of chaos with respect to equilibrium points $Q_{1}$ and $Q_{2}$ (resp. $Q_{3}$ ) are quite different. Therefore, the proposed Definition 2.1 is useful.

\subsection{Simulations of the SCC CNN dynamics}

Now, let us simulate the dynamic behaviors of the 4D SCC equations and the SCC CNNs (96)-(103) with the periodic boundary condition. The parameter groups chosen in the equations and the corresponding simulation results are listed in Table 2 . 
Table 2. Cell parameters and corresponding dynamic properties of the SCC CNNs, where $\left\{\alpha, \beta, D_{1}, D_{2}\right\}=$ $\{10,15,0.01,0.01\}$. The symbols $\Downarrow, \Uparrow, \bigcirc$ and $\bigoplus$ indicate that convergent patterns, divergent patterns, periodic patterns and chaotic patterns are observed near to or far from the corresponding equilibrium points, respectively. The numbers, which are marked by $*$, indicate that the corresponding cell parameters lie on the edge of chaos domain with respect to $Q_{1}$ or $Q_{2}$ (resp. $\left.Q_{3}\right)$.

\begin{tabular}{cccccc}
\hline No. & $a$ & $b$ & Equilibrium Point & Eigenvalue & Pattern \\
\hline $1^{*}$ & -1 & 0.6 & $0.3239,0,-0.3239,0.9718$ & $-10.0000,-0.0211 \pm 3.6814 \mathrm{i},-9.9299$ & $\bigcirc$ \\
2 & -1 & 0.48 & $0.3239,0,-0.3239,0.9718$ & $-10.0000,0.0041 \pm 3.6572 \mathrm{i},-9.3073$ & $\oplus \bigcirc$ \\
3 & -1 & 0 & $0.3239,0,-0.3239,0.9718$ & $-10.0000,0.1218 \pm-3.5017 \mathrm{i},-6.8512$ & $\bigoplus \bigcirc$ \\
4 & -1 & -0.5 & $0.3239,0,-0.3239,0.9718$ & $-10.0000,-0.0002 \pm 3.6615 \mathrm{i},-9.4109$ & $\oplus \Uparrow$ \\
5 & -1 & -0.85 & $0.3239,0,-0.3239,0.9718$ & $-10.0000,0.0936 \pm 2.4924 \mathrm{i},-2.0282$ & $\bigcirc \Uparrow$ \\
$6 *$ & -1 & -0.9 & $0.3239,0,-0.3239,0.9718$ & $-10.0000,-0.0170 \pm 2.3471 \mathrm{i},-1.5268$ & $\Downarrow \Uparrow$ \\
$7^{*}$ & -1.3 & 0.3 & $0.4523,0,-0.4523,1.3568$ & $-10.0000,-0.0161 \pm 3.6768 \mathrm{i},-9.8026$ & $\bigcirc \Uparrow$ \\
8 & -1.3 & -0.1 & $0.5073,0,-0.5073,1.5218$ & $-10.0000,0.0833 \pm 3.5623 \mathrm{i},-7.5973$ & $\bigcirc$ \\
9 & -1.3 & -0.85 & $0.1924,0,-1.3606,4.0819$ & $-10.0000,0.1924 \pm 2.7136 \mathrm{i},-2.7334$ & $\Uparrow$ \\
10 & -1.3 & -0.90 & $1.8639,0,-1.8639,5.5917$ & $-10.0000,0.1173 \pm 2.5340 \mathrm{i},-2.1622$ & $\bigoplus \Uparrow$ \\
11 & -1.3 & -0.93 & $2.5090,0,-2.5090,7.5271$ & $-10.0000,0.0305 \pm 2.4014 \mathrm{i},-1.7237$ & $\bigcirc \Uparrow$ \\
$12^{*}$ & -1.3 & -0.94 & $2.8670,0,-2.8670,8.6010$ & $-10.0000,-0.0111 \pm 2.3532 \mathrm{i},-1.5500$ & $\Downarrow$ \\
$13^{*}$ & -2 & -0.94 & $8.7041,0,-8.7041,26.1124$ & $-10.0000,-0.0018 \pm 2.3633 \mathrm{i},-1.5875$ & $\Downarrow$ \\
14 & -2 & -0.935 & $8.0630,0,-8.0630,24.1889$ & $-10.0000,0.0205 \pm 2.3892 \mathrm{i},-1.6806$ & $\bigcirc$ \\
15 & -2 & -0.8 & $2.8670,0,-2.8670,8.6010$ & $-10.0000,0.2332 \pm 2.9028 \mathrm{i},-3.3738$ & $\oplus \Uparrow$ \\
16 & -2 & -0.7 & $2.0313,0,-2.0313,6.0939$ & $-10.0000,0.2370 \pm 3.1260 \mathrm{i},-4.2754$ & $\Uparrow$ \\
17 & -2 & 0 & $0.8535,0,-0.8535,2.5604$ & $-10.0000,0.0024 \pm 3.6589 \mathrm{i},-9.3478$ & $\bigcirc$ \\
$18^{*}$ & -2 & 0.1 & $0.8069,0,-0.8069,2.4207$ & $-10.0000,-0.0244 \pm 3.6844 \mathrm{i},-10.0166$ & $\bigcirc$ \\
$19^{*}$ & 1 & 1.5 & $0,0,0,0$ & $-10.0000,-0.2136 \pm 3.7869 \mathrm{i}$ & $\Downarrow$ \\
$20^{*}$ & 1 & 1 & $0,0,0,0$ & $-10.0000,-0.2527 \pm 3.8010 \mathrm{i}$ & $\Downarrow$ \\
$21^{*}$ & 1 & 0.5 & $0,0,0,0$ & $-10.0000,-0.2829 \pm 3.8097 \mathrm{i}$ & $\Downarrow$ \\
$22^{*}$ & 1 & 0 & $0,0,0,0$ & $-10.0000,-0.3067 \pm 3.8154 \mathrm{i}$ & $\Downarrow$ \\
$23^{*}$ & 1 & -0.5 & $0,0,0,0$ & $-10.0000,-0.3260 \pm 3.8192 \mathrm{i}$ & $\Downarrow \Uparrow$ \\
$24^{*}$ & 1 & -1 & $0,0,0,0$ & $-10.0000,-0.3418 \pm 3.8219 \mathrm{i}$ & $\bigcirc \Downarrow$ \\
$25^{*}$ & 1 & 1.25 & $0,0,0,0$ & $-10.0000,-0.3551 \pm 3.8237 \mathrm{i}$ & $\Downarrow \Uparrow$ \\
\hline & & & & &
\end{tabular}

The numerical simulations show that the qualitative behaviors of the SCC equations and the SCC CNNs are similar if the diffusion parameters $D_{1}$ and $D_{2}$ are small enough (e.g. $\left.\leq 0.01\right)$.

Roughly speaking, if the parameters $a$ and $b$ are selected in the edge of chaos (red) of the equilibrium $Q_{2}$ shown at the top in Fig. 12(b) [resp. the locally active and unstable domain (green) of $Q_{1}$ given in Fig. 12(a)], the dynamic behaviors of the corresponding SCC CNNs can exhibit oscillation (see Table 2).

If the parameters $a$ and $b$ are chosen in the intersection of the locally active and unstable domains of $Q_{1}$ and $Q_{2}$ shown in Figs. 12(a) and 12(b), the corresponding SCC CNNs may display successively oscillatory, divergent, chaotic and oscillatory patterns. In most cases, if the parameters $a$ and $b$ are located in the locally active domain (green), which are near the bottom edge of chaos shown in Fig. 12(b), chaotic patterns can be generated.

On the other hand, if the parameters $a$ and $b$ are selected in the edge of chaos (red) of the equilibrium $Q_{1}$ in the left area in Fig. 12(a), in which no nonzero equilibrium $Q_{2}, Q_{3}$ exist, then for most cases, divergent patterns are observed via simulations. Oscillatory patterns or divergent patterns may also coexist with divergent patterns by choosing different initial conditions (see items 19-25 listed in Table 2).

Figures 13(a)-13(c) exhibit, respectively, the chaotic trajectories and periodic trajectories, which are generated via the SCC equations and numbered 


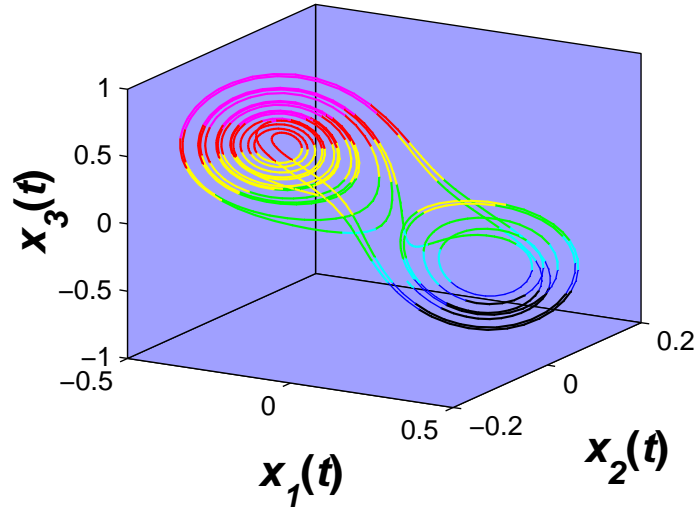

(a)

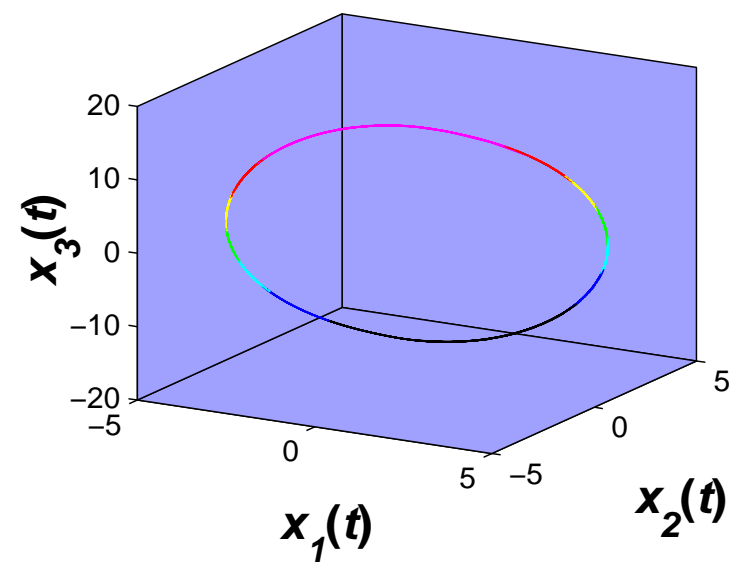

(b)

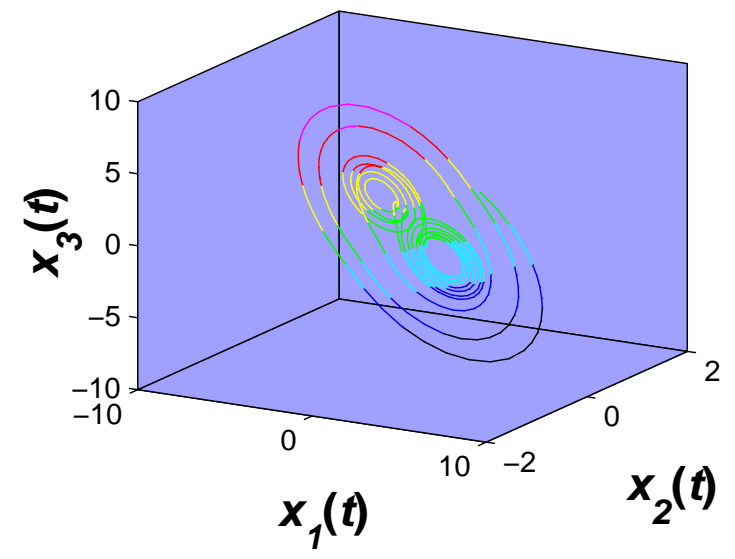

(c)

Fig. 13. Trajectories of the state variables $x_{1}, x_{2}$ and $x_{3}$ of the SCC equations. The chaotic trajectory and the periodic trajectory generated by the parameter set No. 3 listed in Table 2, with the initial condition $\left(x_{1}(0), x_{2}(0), x_{3}(0)\right)$ : (a) $(-0.1630,-0.0265,0.0602,-0.4275)$, and (b) $(-0.3342$, $1.2220,12.5481,-2.2370)$. (c) The chaotic trajectory generated by the parameter set No. 10 listed in Table 2 with the initial condition $(1,1,1,1)$.

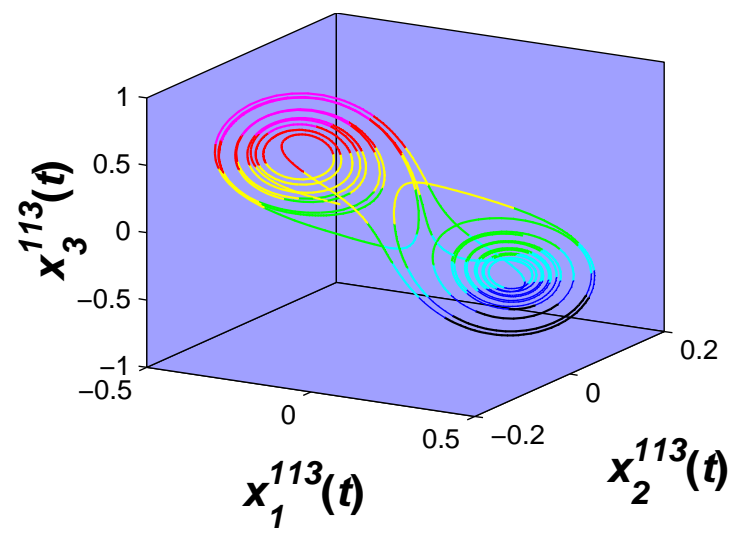

(a)

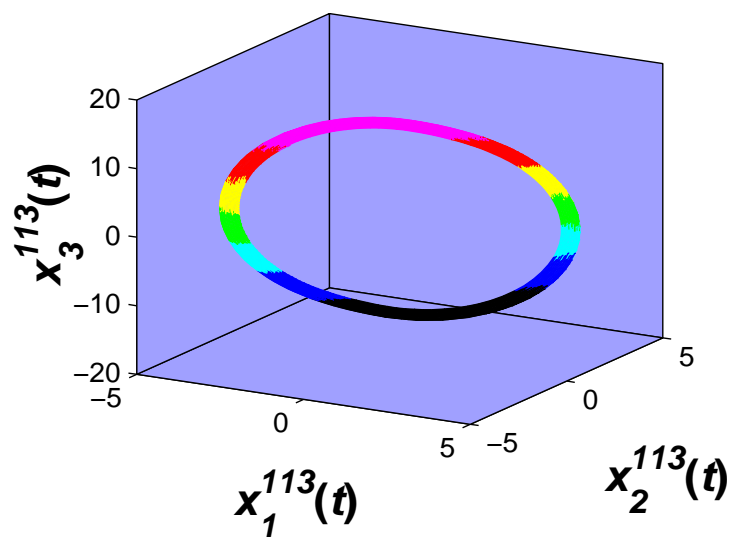

(b)

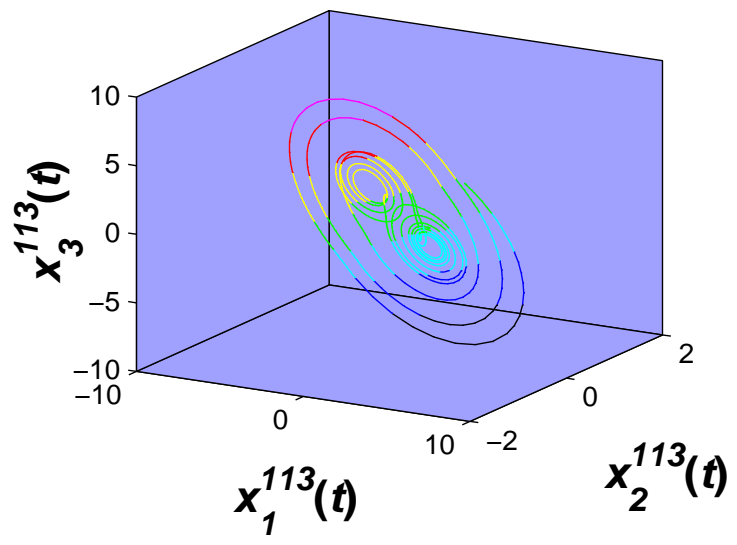

(c)

Fig. 14. Trajectories of the components of the states $\mathbf{X}_{1}, \mathbf{X}_{2}$ and $\mathbf{X}_{3}$ of the SCC CNNs. The trajectories of the SCC CNNS generated by the parameter set No. 3 listed in Table 2, with different initial conditions: (a) The chaotic trajectory, and (b) The trajectory converges to a limit cycle. (c) The chaotic trajectories generated by the parameter set No. 10 listed in Table 2. 
by 3 and 10 listed in Table 2 with different initial conditions.

Now, let us simulate their corresponding $15 \times 15$ SCC CNNs with periodic boundary conditions.

- The patterns generated by the parameter group No. 3. The parameter group is located in the locally active domain and nearby the oblique edge of chaos with respect to the equilibrium point $Q_{2}$ [see Fig. 12(b)]. Figures 14(a) and 14(b) show the trajectories of the components of the states $\mathbf{X}_{1}, \mathbf{X}_{\mathbf{2}}$ and $\mathbf{X}_{\mathbf{3}}$ of the SCC CNNs are very similar to those of the original SCC equations [see Figs. 13(a) and 13(b)]. The graphs of the time evolution of the patterns of the local state variables of the SCC CNN over the time interval $[0,40]$ are shown in Figs. 15 and 16, respectively. The diffusion parameters $D_{1}, D_{2}$ are selected as 0.01. The initial condition is chosen as follows.

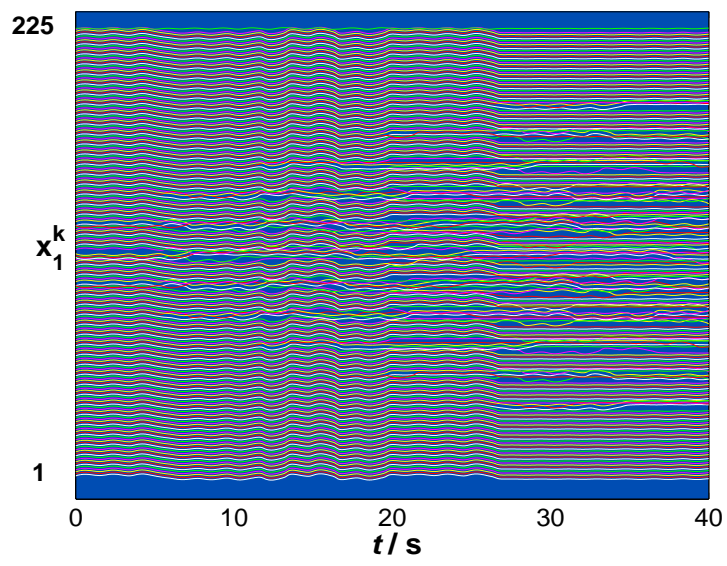

(a)

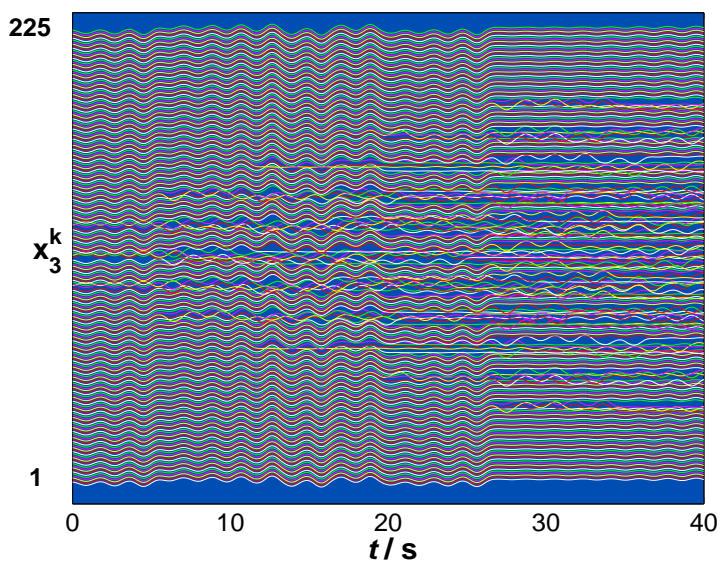

(c)
For Figs. 14(a) and 15:

$$
\begin{aligned}
& x_{1 i, j}(0)= \begin{cases}-0.1630, & \text { if } 7 \leq i \leq 9, j=8 \\
& \text { or } 7 \leq j \leq 9, i=8 \\
0.1630, & \text { otherwise }\end{cases} \\
& x_{2 i, j}(0)= \begin{cases}-0.0265, & \text { if } 7 \leq i \leq 9, j=8 \\
& \text { or } 7 \leq j \leq 9, i=8 ; \\
0.0265, & \text { otherwise. }\end{cases} \\
& x_{3 i, j}(0)= \begin{cases}0.0602, & \text { if } 7 \leq i \leq 9, j=8 \\
& \text { or } 7 \leq j \leq 9, i=8 \\
-0.0602, & \text { otherwise }\end{cases} \\
& x_{4 i, j}(0)= \begin{cases}-0.4275, & \text { if } 7 \leq i \leq 9, j=8 \\
& \text { or } 7 \leq j \leq 9, i=8 \\
0.4275, & \text { otherwise }\end{cases}
\end{aligned}
$$

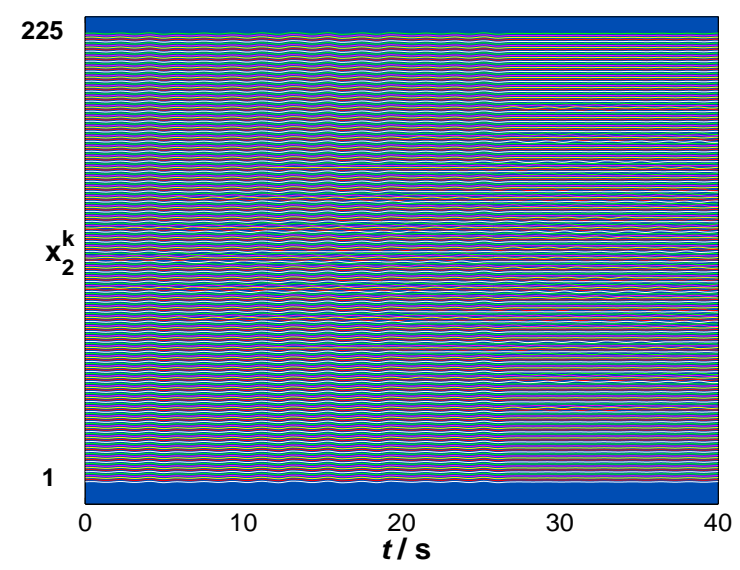

(b)

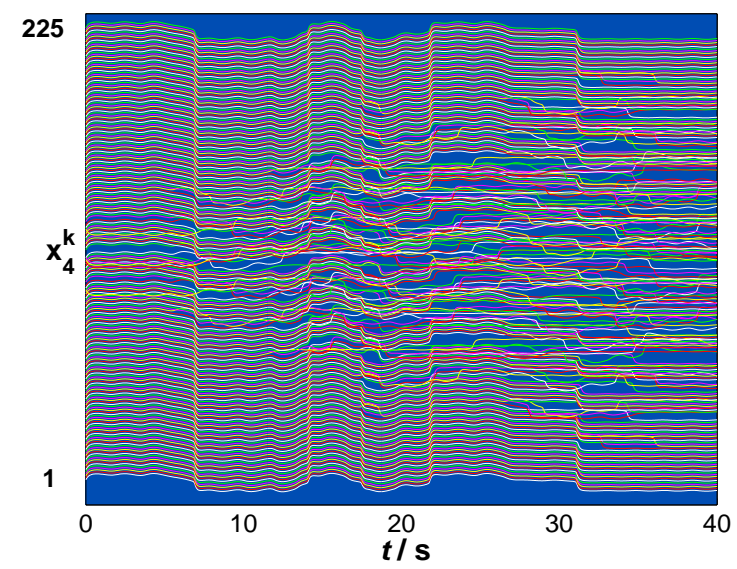

(d)

Fig. 15. Graphs of the time evolution of the state variables $x_{1}^{k^{\prime}} s, x_{2}^{k^{\prime}} s, x_{3}^{k^{\prime}} s$ and $x_{4}^{k^{\prime}} s$ of SCC CNNs generated by the parameter set No. 3 listed in Table 2 . 


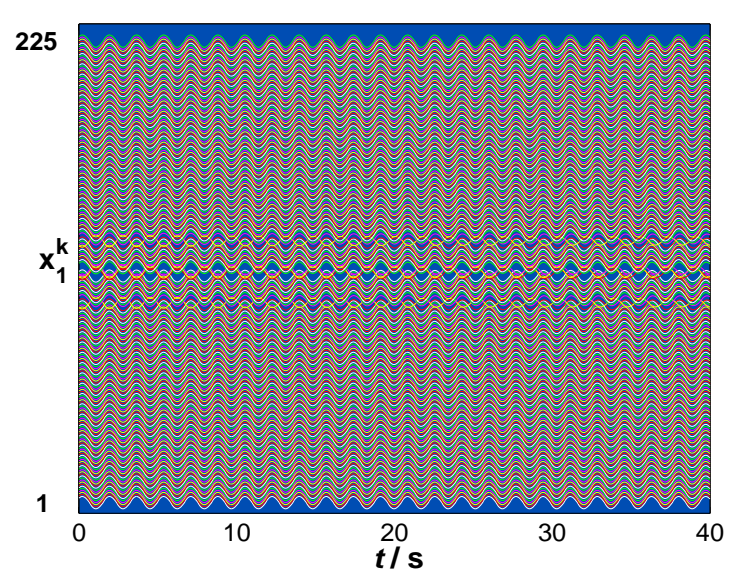

(a)

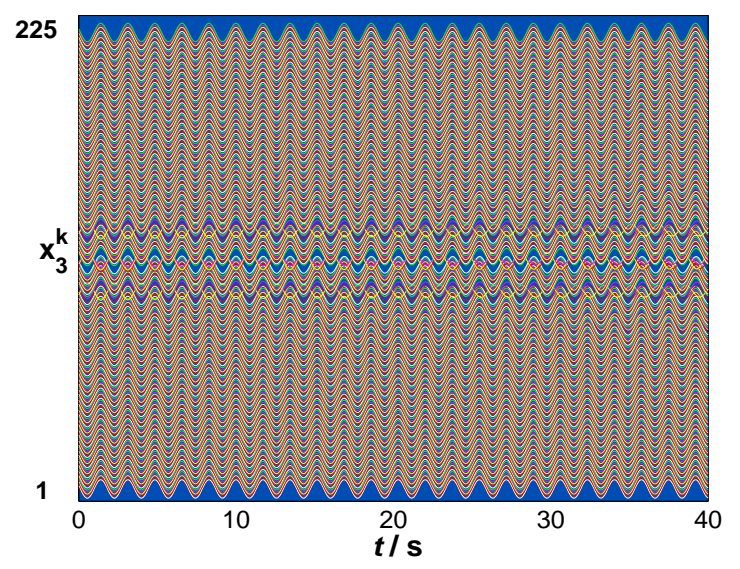

(c)

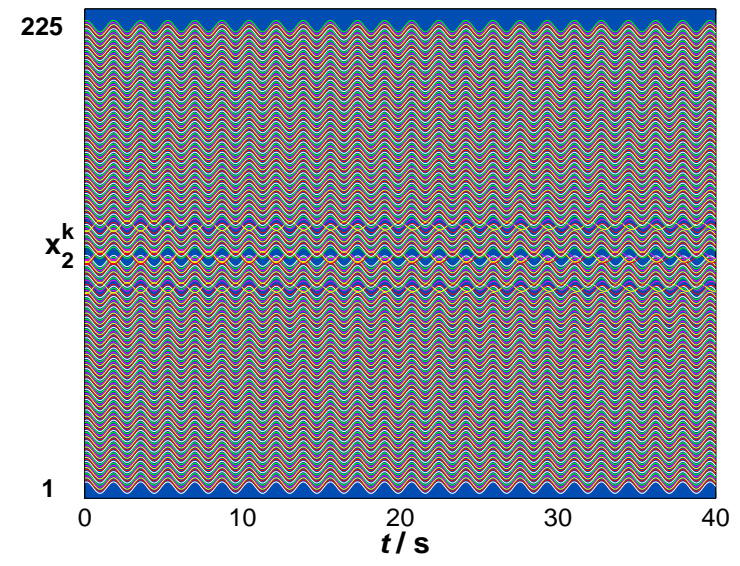

(b)

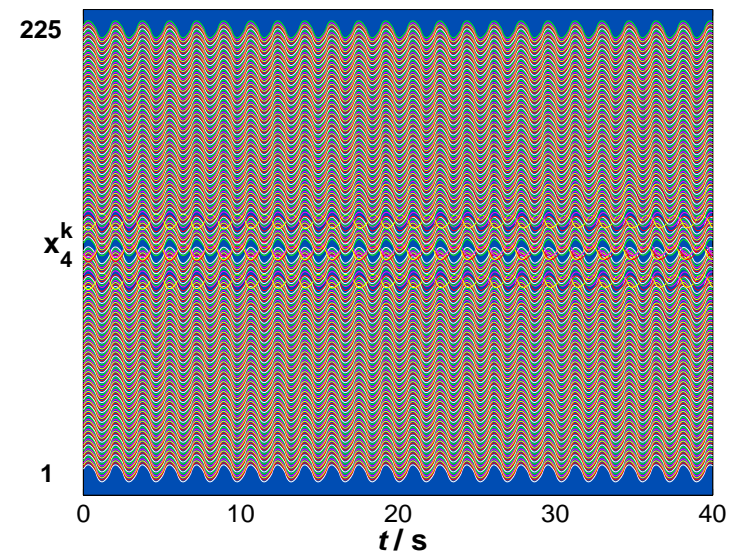

(d)

Fig. 16. Graphs of the time evolution of the state variables $x_{1}^{k^{\prime}} s, x_{2}^{k^{\prime}} s, x_{3}^{k^{\prime}} s$ and $x_{4}^{k^{\prime}} s$ of SCC CNNs generated by the parameter set No. 3 listed in Table 2, with different initial conditions.

For Fig. 14(b) and Fig. 16:

$$
\begin{aligned}
& x_{1 i, j}(0)= \begin{cases}-0.3342, & \text { if } 7 \leq i \leq 9, j=8 \\
0.3342, & \text { or } 7 \leq j \leq 9, i=8 ; \\
\text { otherwise }\end{cases} \\
& x_{2 i, j}(0)= \begin{cases}1.2220, & \text { if } 7 \leq i \leq 9, j=8 \\
-1.2220, & \text { or } 7 \leq j \leq 9, i=8 ;\end{cases} \\
& x_{3 i, j}(0)= \begin{cases}12.5481, & \text { if } 7 \leq i \leq 9, j=8 \\
-12.5481, & \text { otherwise. }\end{cases} \\
& x_{4 i, j}(0)= \begin{cases}-2.2370, & \text { if } 7 \leq i \leq 9, j=8 \\
2.2370, & \text { or } 7 \leq j \leq 9, i=8 ;\end{cases}
\end{aligned}
$$

- The patterns generated by the parameter group No. 10. The parameter group is located in the locally active domain and nearby the horizontal edge of chaos with respect to the equilibrium point $Q_{2}$ [see Fig. 12(b)]. Figure 14(c) shows the trajectories of the components of the states $\mathbf{X}_{1}, \mathbf{X}_{\mathbf{2}}$ and $\mathbf{X}_{\mathbf{3}}$ of the SCC CNNs are very similar to those of the original SCC equations [see Fig. 13(c)]. The graphs of the time evolution of the chaotic patterns of the local state variables of the SCC CNN over the time interval $[0,40]$ are shown in Fig. 17. The diffusion parameters $D_{1}$, $D_{2}$ are selected as 0.01 . The initial condition is chosen as follows.

$$
\begin{aligned}
& x_{1 i, j}(0)= \begin{cases}1, & \text { if } 7 \leq i \leq 9, j=8 \\
-1, & \text { or } 7 \leq j \leq 9, i=8 ;\end{cases} \\
& x_{2 i, j}(0)= \begin{cases}1, & \text { if } 7 \leq i \leq 9, j=8 \\
-1, & \text { or } 7 \leq j \leq 9, i=8 ;\end{cases} \\
&
\end{aligned}
$$




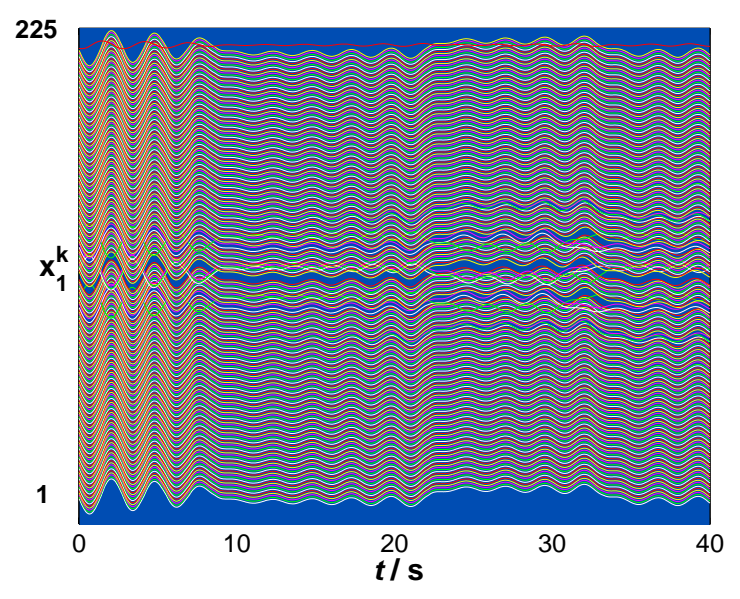

(a)

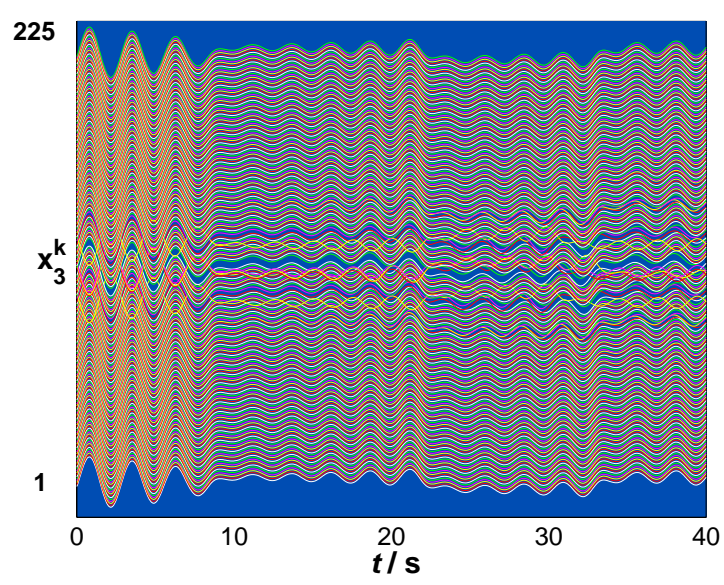

(c)

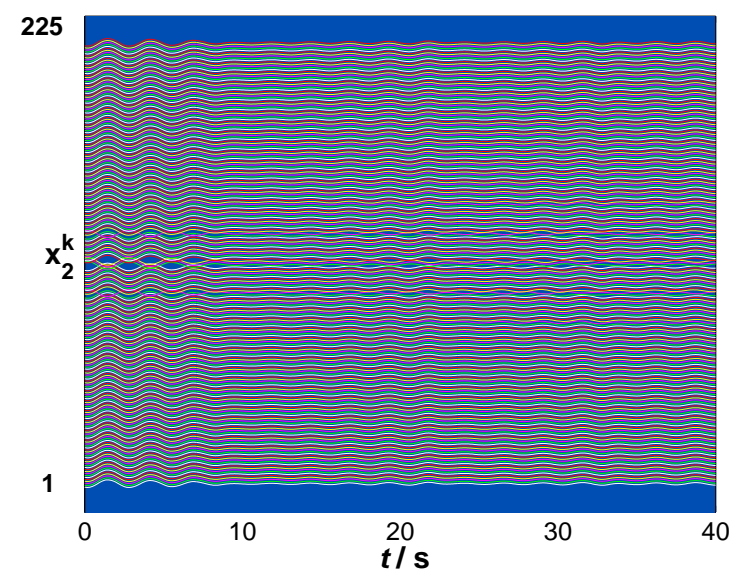

(b)

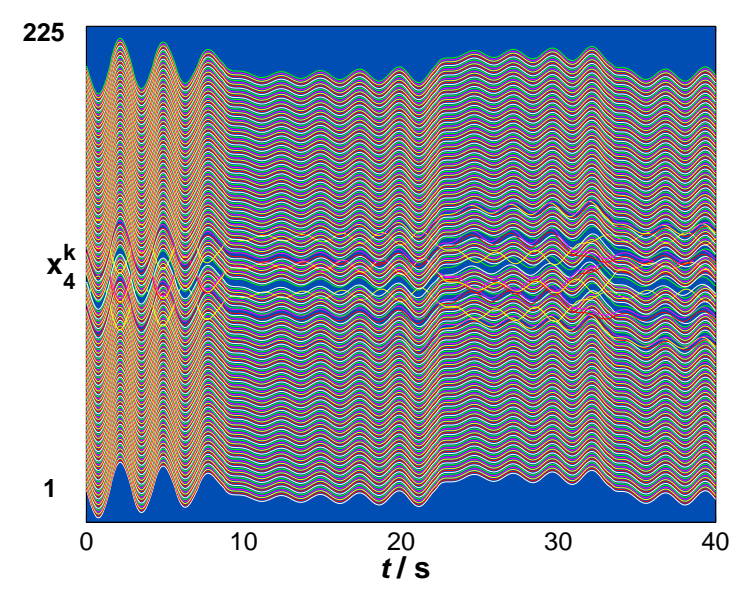

(d)

Fig. 17. Graphs of the time evolution of the state variables $x_{1}^{k^{\prime}} s, x_{2}^{k^{\prime}} s, x_{3}^{k^{\prime}} s$ and $x_{4}^{k^{\prime}} s$ of SCC CNNs generated by the parameter set No. 10 listed in Table 2 .

$$
\begin{gathered}
x_{3 i, j}(0)= \begin{cases}1, & \text { if } 7 \leq i \leq 9, j=8 \\
-1 & \text { or } 7 \leq j \leq 9, i=8\end{cases} \\
x_{4 i, j}(0)= \begin{cases}1, & \text { if } 7 \leq i \leq 9, j=8 \\
-1, & \text { or } 7 \leq j \leq 9, i=8\end{cases}
\end{gathered}
$$

- Now let us continue with the state variables of the above SCC CNN with the chessboard-shaped initial conditions given by

$$
x_{k i, j}(0)= \begin{cases}1, & \text { if } i+j \text { is an even number } \\ -1, & \text { otherwise }\end{cases}
$$

where $k=1,2,3,4$. The evolution of chaotic patterns of the state variable $x_{1 i, j}$ over the time interval $[0,120]$ are shown in Fig. 19; the evolution of chaotic patterns of the state variables $x_{1 i, j}, x_{2 i, j}, x_{3 i, j}$ and $x_{4 i, j}$ at the time 30, 52.9, 72.5, 89.7 and 105 are shown in Fig. 20, in which the pseudo-color code for the components $x_{k i, j}^{\prime} s$ of the solution of the CNN state equation are chosen as similar to that shown in Fig. 7 in [Min et al., 2000b]. These figures exhibit once again that smoothed Chua's circuit CNNs are the machines generating complex patterns.

- The patterns generated by the parameter group No. 24. The parameter group is located in the the edge of chaos with respect to the equilibrium point $Q_{1}=(0,0,0,0)$ [see Fig. 12(a)]. The graphs of the time evolution of the limit cycle patterns of the local state variables of the SCC CNN over the time interval $[0,40]$ are shown in Fig. 18. 


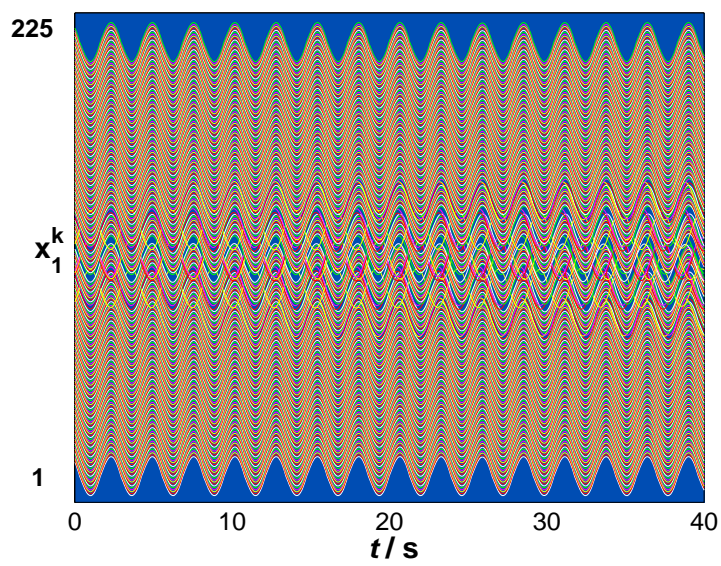

(a)

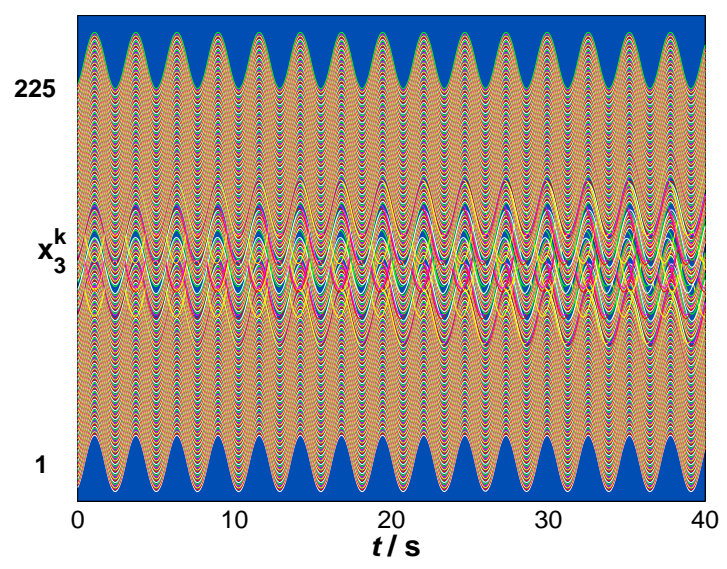

(c)

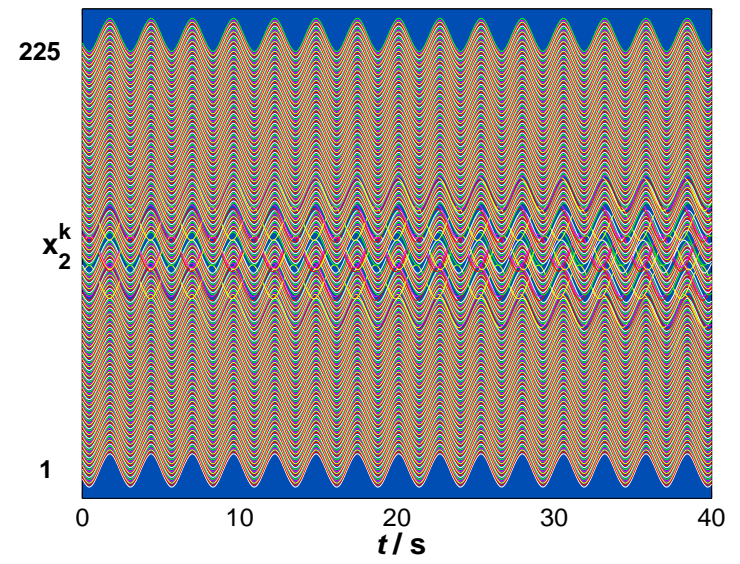

(b)

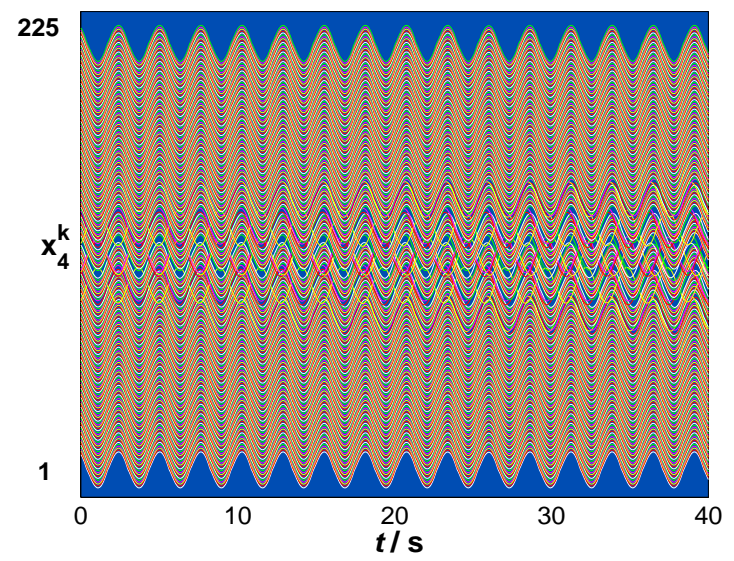

(d)

Fig. 18. Graphs of the time evolution of the state variables $x_{1}^{k^{\prime}} s, x_{2}^{k^{\prime}} s, x_{3}^{k^{\prime}} s$ and $x_{4}^{k^{\prime}} s$ of SCC CNN's generated by the parameter set No. 24 listed in Table 2.

The diffusion parameters $D_{1}, D_{2}$ are selected as 0.01 . The initial condition is chosen as follows.

$$
\begin{aligned}
& x_{1 i, j}(0)= \begin{cases}-14.3159, & \text { if } 7 \leq i \leq 9, j=8 \\
& \text { or } 7 \leq j \leq 9, i=8 \\
14.3159, & \text { otherwise }\end{cases} \\
& x_{2 i, j}(0)= \begin{cases}2.5546, & \text { if } 7 \leq i \leq 9, j=8 \\
& \text { or } 7 \leq j \leq 9, i=8 \\
-2.5546, & \text { otherwise }\end{cases} \\
& x_{3 i, j}(0)=\left\{\begin{aligned}
26.7470, & \text { if } 7 \leq i \leq 9, j=8 \\
& \text { or } 7 \leq j \leq 9, i=8 \\
-26.7470, & \text { otherwise }
\end{aligned}\right. \\
& x_{4 i, j}(0)= \begin{cases}-48.3950, & \text { if } 7 \leq i \leq 9, j=8 \\
& \text { or } 7 \leq j \leq 9, i=8 \\
48.3950, & \text { otherwise }\end{cases}
\end{aligned}
$$

\subsection{Concluding remarks}

Based on the local activity principle of CNN cells presented by [Chua, 1977, 1999], analytical criteria of the local activity for two-port CNNs with four state variables are set up. The analytical criteria consist of Theorems 4.1-4.4, which can be implemented by a computer program to produce bifurcation diagrams for general corresponding CNNs.

A two-port SCC CNN is introduced and the bifurcation diagrams of the SCC CNN are calculated. Although the analytical criteria are much more complex than those of the one-port CNN [Min et al., 2000b], the bifurcation diagrams of the twoport SCC CNN and the one-port SCC CNN calculated via our criteria are the same (see Figs. 12(a), 12(b) and Figs. 5(a) and 5(c) in [Min et al., 2000b]).

The numerical simulations exhibit the extremely complex behaviors possible in the SCC CNN, which are similar to the SCC Eqs. (96)-(99), 

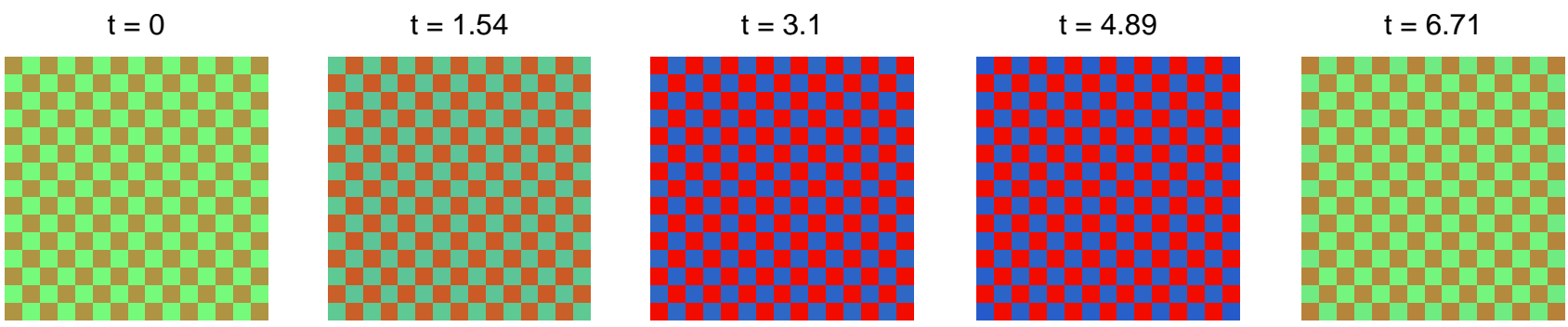

$t=8.39$

$t=10.1$

$t=13.5$

$t=16.9$

$t=19.9$
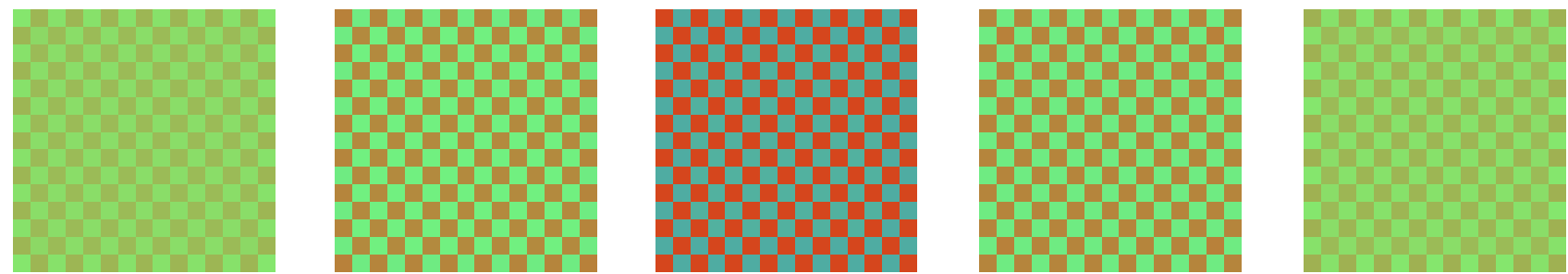

$t=22.4$

$t=24.4$
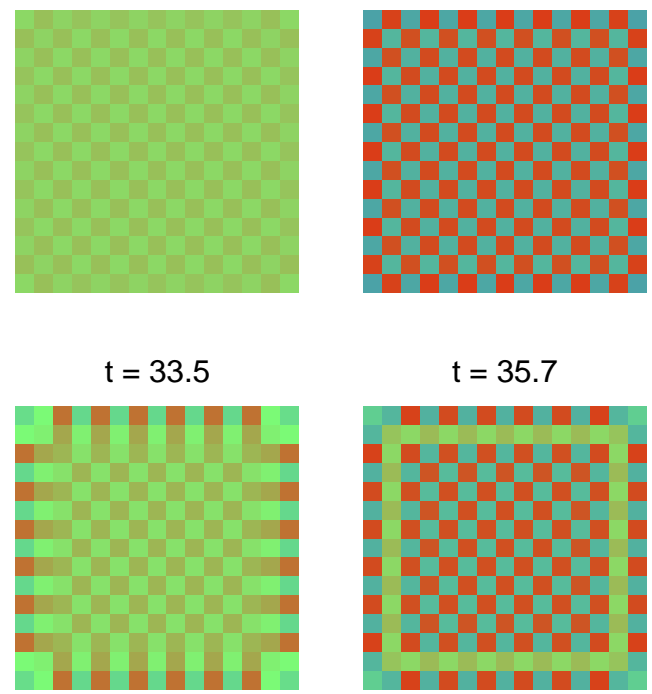

$\mathrm{t}=44$

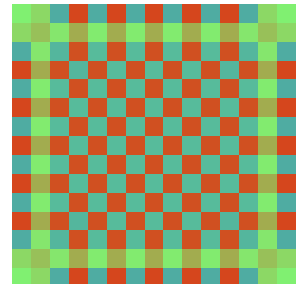

$t=35.7$

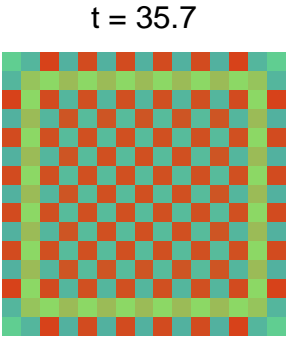

$t=45.6$

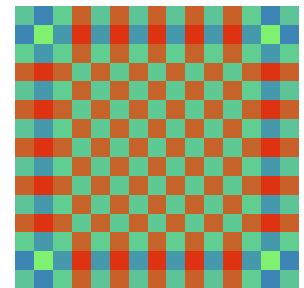

$t=27.8$

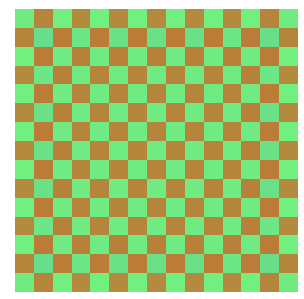

$t=37.3$

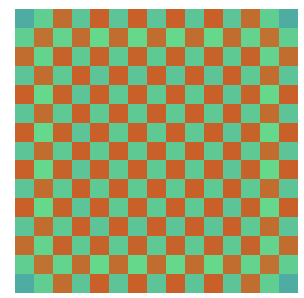

$\mathrm{t}=47$

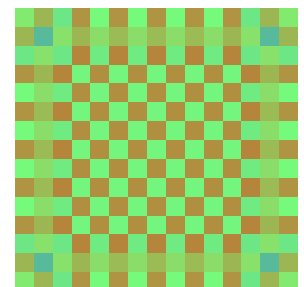

$t=30.5$

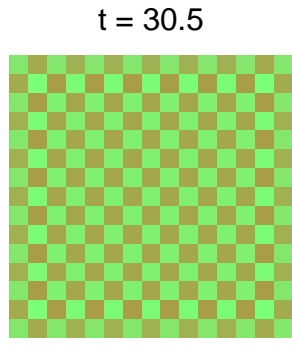

$t=39.4$

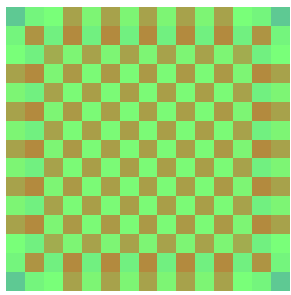

$t=49.2$

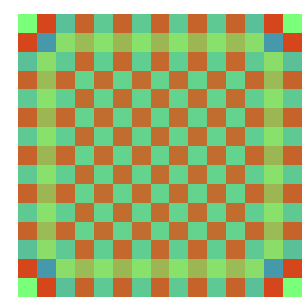

$t=31.8$

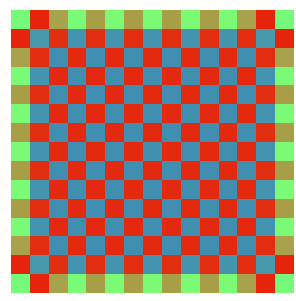

$\mathrm{t}=41.7$

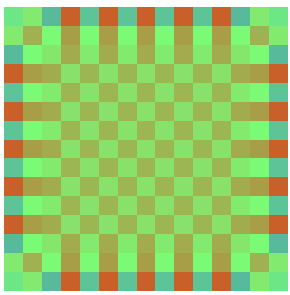

$t=50.7$

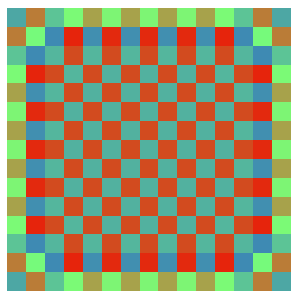

Fig. 19. Evolution of chaotic patterns of the state variable $x_{1 i, j}$ of the $15 \times 15$ SCC CNN generated by the parameter set No. 10 listed in Table 2 over the time interval [0,120]. 

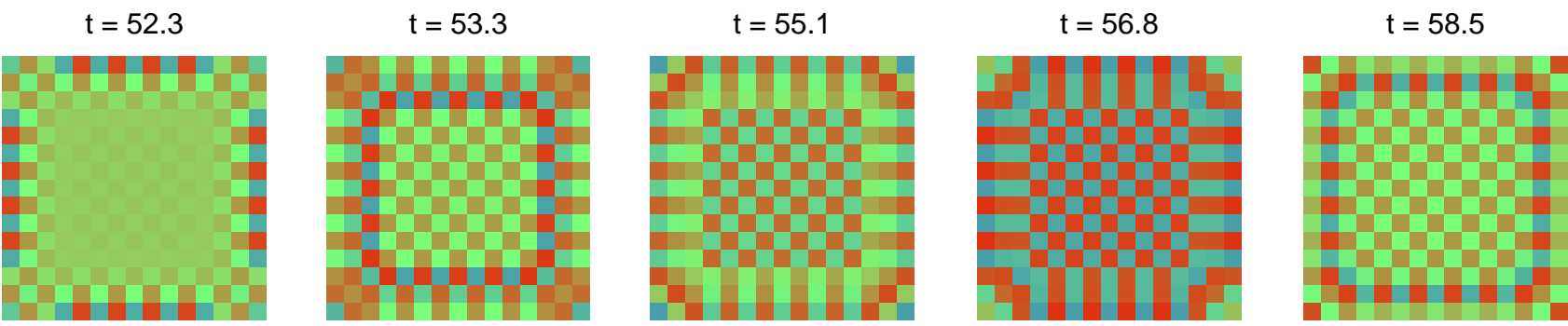

$t=60.2$

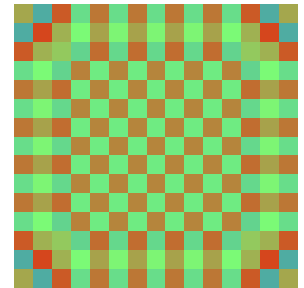

$t=67.4$

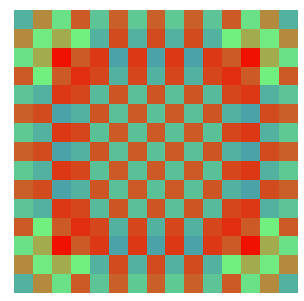

$\mathrm{t}=74.8$

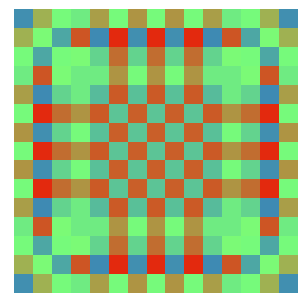

$\mathrm{t}=81.2$

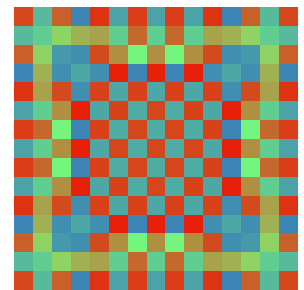

$t=61.4$

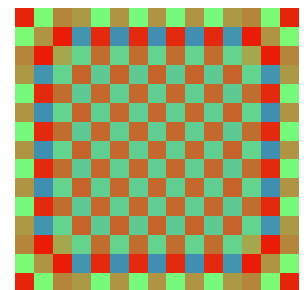

$t=68.6$

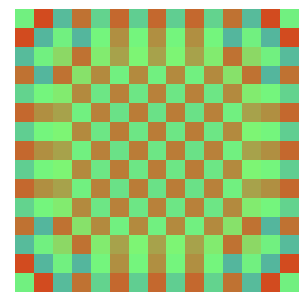

$\mathrm{t}=76.6$

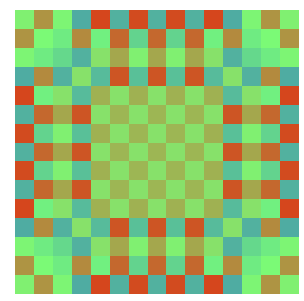

$\mathrm{t}=82.3$

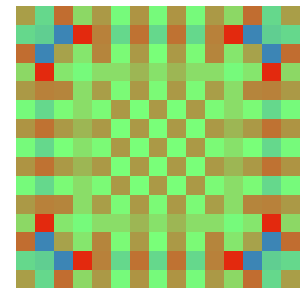

$t=63.2$

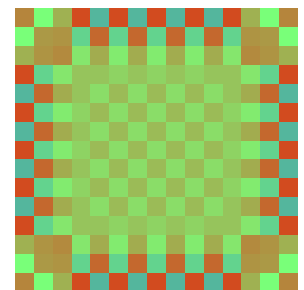

$t=70.2$

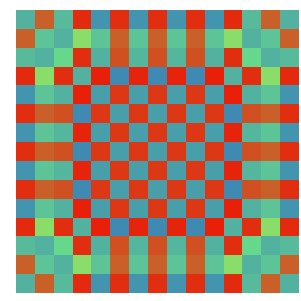

$\mathrm{t}=77.7$

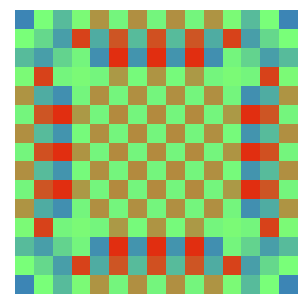

$\mathrm{t}=83.7$

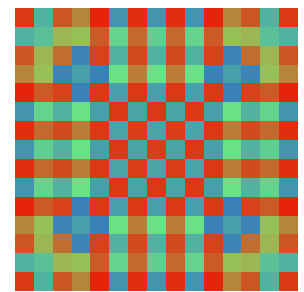

$t=64.9$

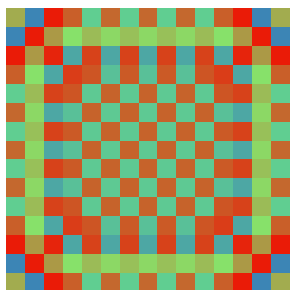

$t=71.8$

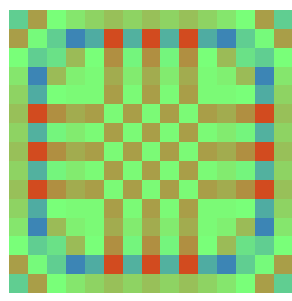

$t=79$

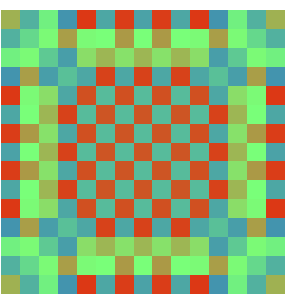

$t=84.9$

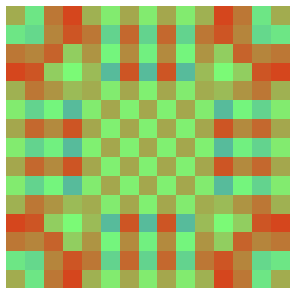

$t=65.9$

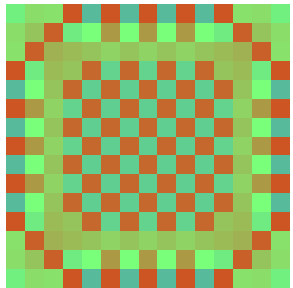

$t=73.3$

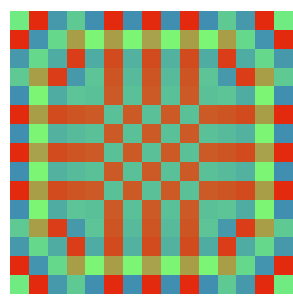

$t=79.9$

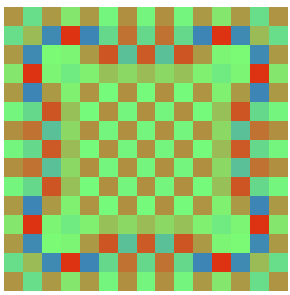

$t=86.2$

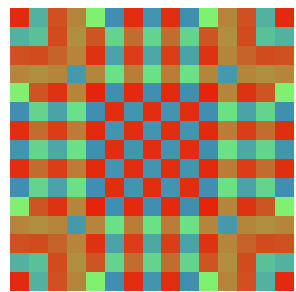

Fig. 19. (Continued) 

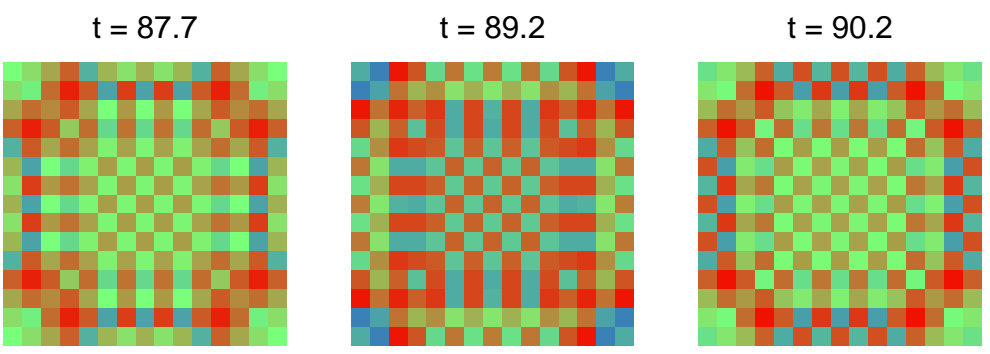

$t=93.7$

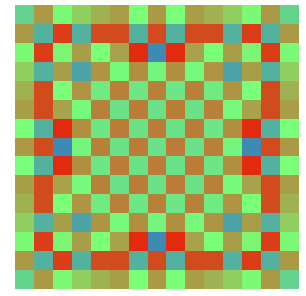

$t=99.6$

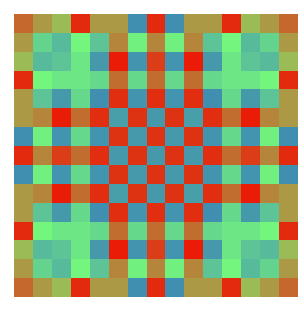

$t=105.5$

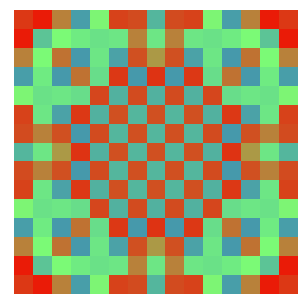

$t=112.2$

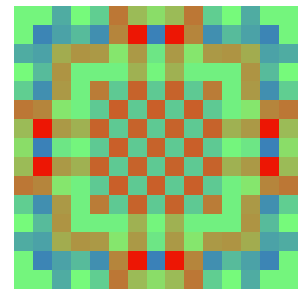

$t=94.9$

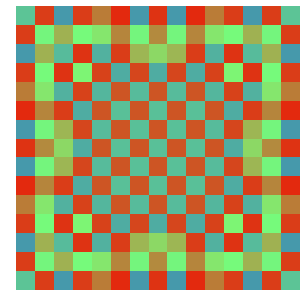

$t=101$

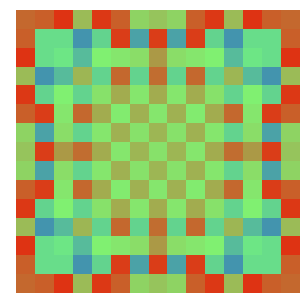

$t=106.8$

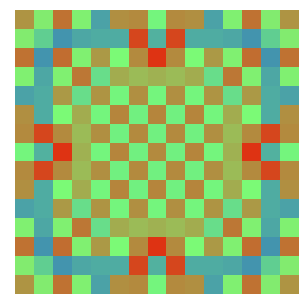

$t=113.5$

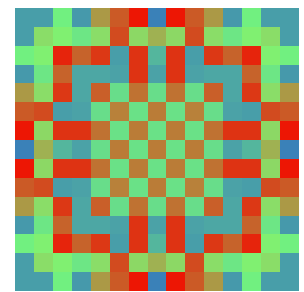

$t=95.9$

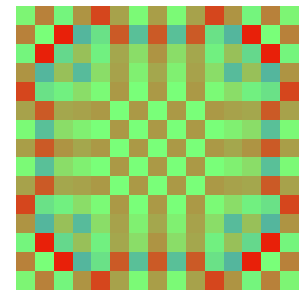

$t=102.2$

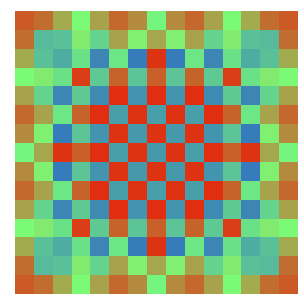

$t=108.5$

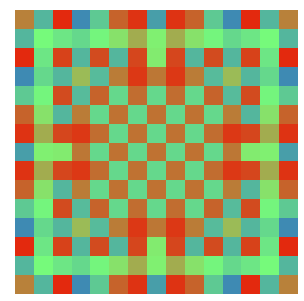

$t=114.4$

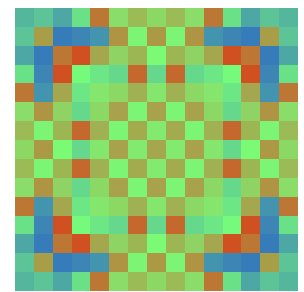

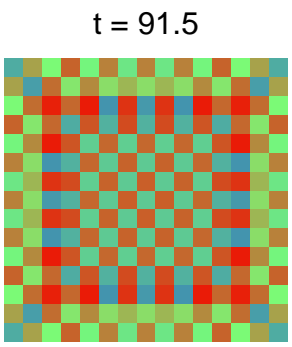

$t=97.4$

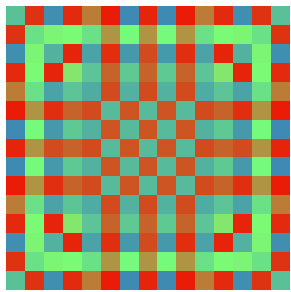

$t=103.4$

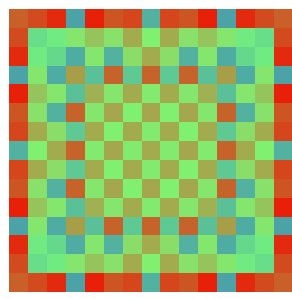

$t=109.6$

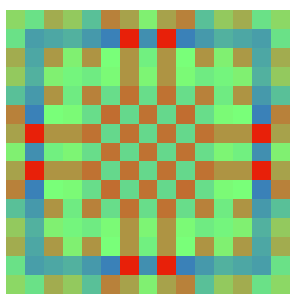

$t=115.1$

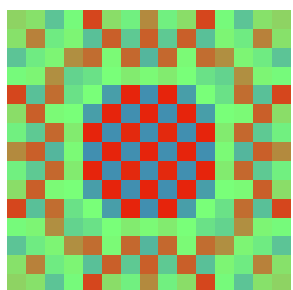

$t=92.7$

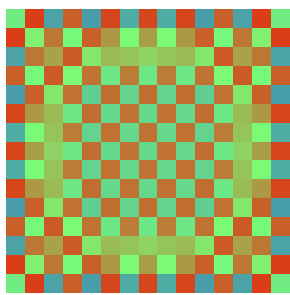

$t=98.7$

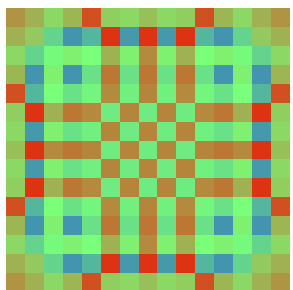

$t=104.4$

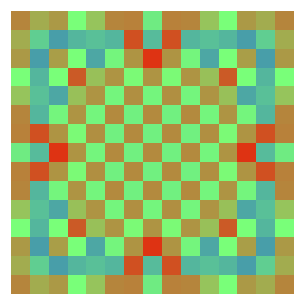

$t=110.9$

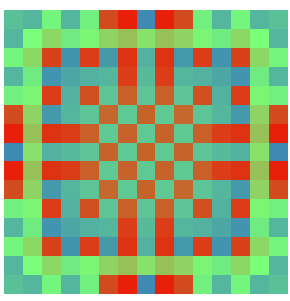

$t=120$

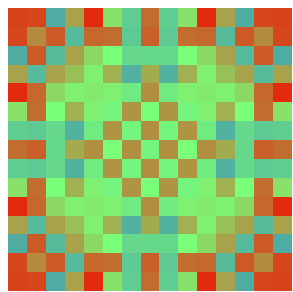

Fig. 19. (Continued) 
$t=30$

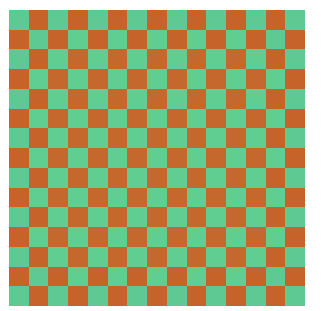

$x_{1} \mathbf{i , j}$

$t=52.9$

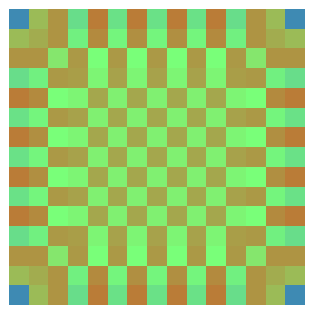

$x_{1} \mathbf{i , j}$

$t=72.5$

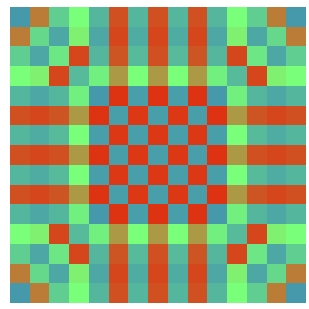

$x_{1} \mathbf{i}, \mathbf{j}$

$\mathrm{t}=89.7$

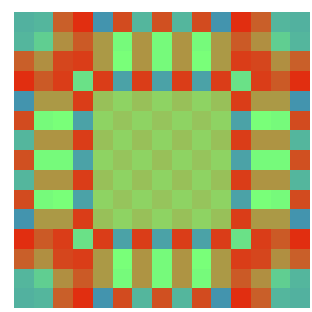

$\mathbf{x}_{1 \mathrm{i}, \mathrm{j}}$

$t=105$

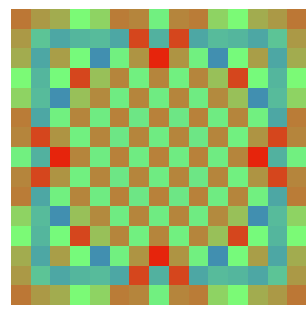

$x_{1} \mathbf{i , j}$ $t=30$

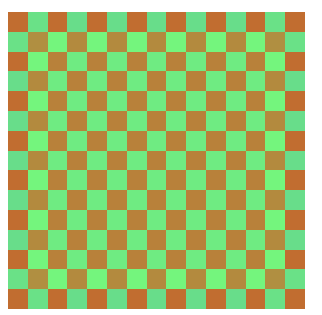

$\mathbf{x}_{2} \mathbf{i , j}$

$t=52.9$

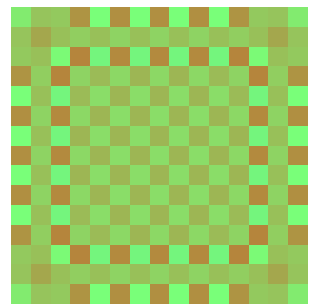

$x_{2} \mathbf{i , j}$

$t=72.5$

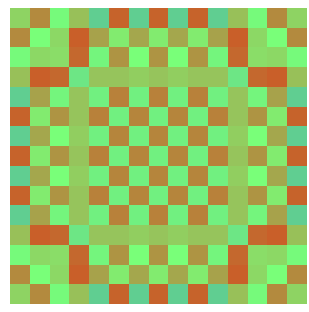

$x_{2} \mathrm{i}, \mathrm{j}$

$\mathrm{t}=89.7$

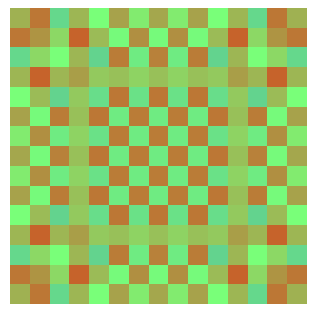

$\mathbf{x}_{\mathbf{2} \mathbf{i}, \mathbf{j}}$

$t=105$

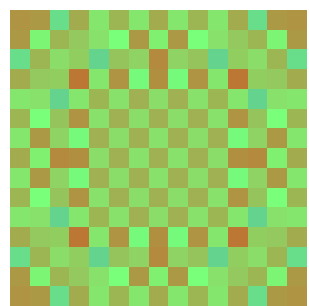

$x_{2} \mathbf{i , j}$ $t=30$

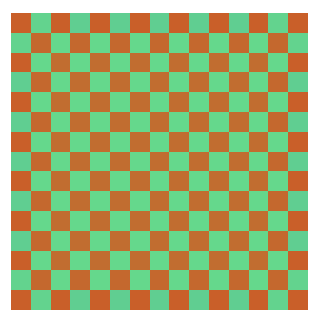

$\mathbf{x}_{3 \mathbf{i}, \mathbf{j}}$

$\mathrm{t}=52.9$

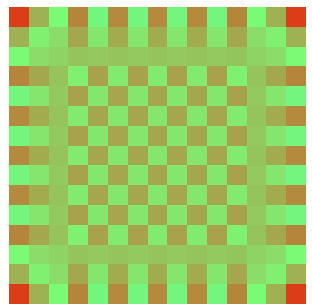

$x_{3 \mathbf{i}, j}$

$t=72.5$

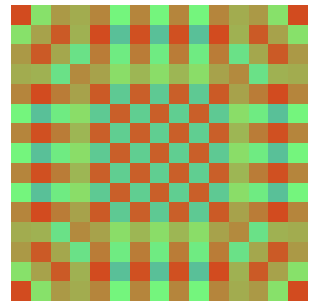

$\mathbf{x}_{3 \mathbf{i}, \mathbf{j}}$

$t=89.7$

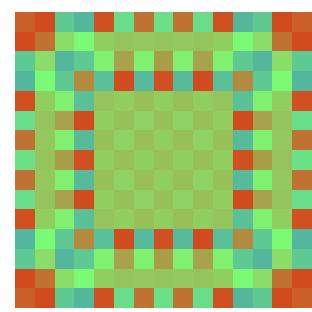

$\mathbf{x}_{\mathbf{3} \mathbf{i}, \mathbf{j}}$

$t=105$

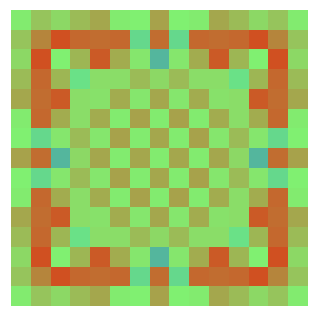

$\mathbf{x}_{\mathbf{3} \mathbf{i}, \mathbf{j}}$ $t=30$

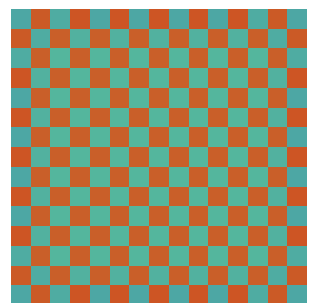

$\mathrm{X}_{4 \mathbf{i}, \mathrm{j}}$

$\mathrm{t}=52.9$

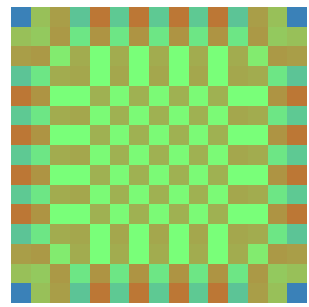

$x_{4 i, j}$

$t=72.5$

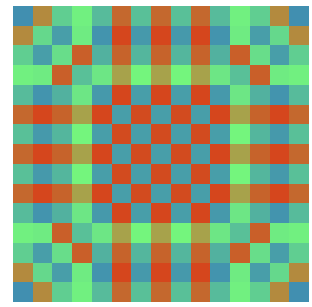

$x_{4 i, j}$

$t=89.7$

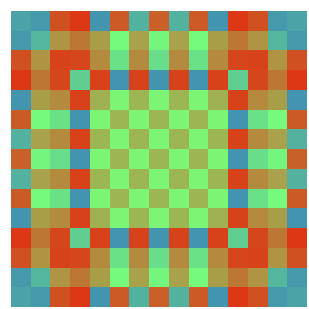

$x_{4 i, j}$

$t=105$

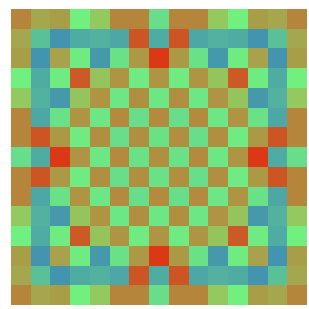

$x_{4 i, j}$

Fig. 20. Evolution of chaotic patterns of the state variables $x_{1 i, j}, x_{2 i, j}, x_{3 i, j}$ and $x_{4 i, j}$ of the $15 \times 15 \mathrm{SCC}$ CNN generated by the parameter set No. 10 listed in Table 2, over the time interval $[0,120]$. 
if the diffusion coefficients of the SCC CNN are small enough. Chaotic patterns, oscillatory patterns, and divergent patterns can be obtained, if the corresponding cell parameters of the SCC CNNs are selected in the locally active and unstable domain or in the edge of chaos domains.

In summary, this paper has demonstrated once again that the local activity theory provides a practical tool for the study of the complex dynamics of some nonlinear systems.

\section{Acknowledgments}

The authors would like to express sincere gratitude to Professor L. O. Chua at the UC Berkeley for directing the first author to study the local activity theory, and for his criticisms and constructive suggestions, which helped improve the presentation of this paper. The authors would also like to thank Professor G. Chen at the City University of Hong Kong for his helpful suggestions. This project is jointly supported by the National Natural Science Foundation of China (Grant No. 0074034) and the Foundation for University Key Teachers by the China Ministry of Education.

\section{References}

Chay, T. R. [1985] "Chaos in a three-variable model of an excitable cell," Physica D16, 223-242.

Chua, L. O. \& Yang, L. [1988a] "Cellular neural networks: Theory," IEEE Trans. Circuits Syst. 35, $1257-1272$.

Chua, L. O. \& Yang, L. [1988b] "Cellular neural networks: Applications," IEEE Trans. Circuits Syst. 35, 1273-1290.

Chua, L. O. \& Roska, T. [1990] "Stability of a class of nonreciprocal cellular neural networks," IEEE Trans. Circuits Syst. 37, 1520-1527.

Chua, L. O. \& Wu, C. W. [1992] "On the universe of stable cellular neural networks," Int. J. Circuit Th. Appl. 20, 497-517.

Chua, L. O. [1995] "Autonomous cellular neural networks: A unified paradigm for pattern formation and active wave propagation," IEEE Trans. Circuits Syst. I, Fundamental Th. Appl. 42(10), 559-577.

Chua, L. O. [1997] "CNN: A vision of complexity," Int. J. Bifurcation and Chaos 7(10), 2219-2425.

Chua, L. O. [1999] "Passivity and complexity," IEEE Trans. Circuits Syst. I, Fundamental Th. Appl. 46(1), 71-82.

Dogaru, R. \& Chua, L. O. [1998a] "Edge of chaos and local activity domain of FitzHugh-Nagumo equation," Int. J. Bifurcation and Chaos 8(2), 211-257.
Dogaru, R. \& Chua, L. O. [1998b] "Edge of chaos and local activity domain of the Brusselator CNN," Int. J. Bifurcation and Chaos 8(6), 1107-1130.

Dogaru, R. \& Chua, L. O. [1998c] "Edge of chaos and local activity domain of the Gierer-Meinhardt CNN," Int. J. Bifurcation and Chaos 8(12), 2321-2340.

Gili, M. [1994] "Stability of cellular neural networks and delayed neural networks with nonpositive templates and nonmonotonic output functions," IEEE Trans. Circuits Syst. 40, 207-212.

Hodgkin, A. L. \& Huxley, A. F. [1952] "A quantitative description of membrane current and its application to conduction and excitation in nerve," J. Physiol. 117, 500-544.

Hopfield, J. J. [1985] "Neural networks and physical systems with emergent computational abilities," Proc. Nat. Acad. Sci. USA 79, 2554-2558.

Joy, M. P. \& Tavasnoglu, V. [1993] "A new parameter range for the stability of opposite-sign cellular neural networks," IEEE Trans. Circuits Syst. 40, 213-215.

Li, W. \& Min, L. [2000] "A new template of CNN for processing image with disturbed convex objects," $A d v$. Syst. Sci. Appl. 2, 58-62.

Lin, S.-S. \& Shih, C.-W. [1999] "Complete stability for standard cellular neural networks," Int. J. Bifurcation and Chaos 9(5), 909-918.

Min, L., Crounse, K. R. \& Chua, L. O. [1999] " $\Omega$-globally attractive equilibrium points of the CNN," Int. J. Bifurcation and Chaos 9(4), 671-693.

Min, L., Crounse, K. R. \& Chua, L. O. [2000a] "Analytical criteria for local activity and applications to the oregonator CNN," Int. J. Bifurcation and Chaos 10(1), 25-71.

Min, L., Crounse, K. R. \& Chua, L. O. [2000b] "Analytical criteria for local activity of reaction-diffusion CNN with four state variables and applications to the Hodgkin-Huxley equation," Int. J. Bifurcation and Chaos 10(6), 1295-1343.

Min, L. \& Yu, N. [2000] "Analytical criteria for local activity of CNN with two-port and applications to biochemecal model," J. Univ. Sci. Tech. Beijing 7(4), 305-314.

Orzo, L. et al. [1996] "CNN model of the featurelinked synchronized activities in the visual thalamocortical system," Proc. 1996 Fourth IEEE Int. Workshop on Cellular Neural Networks and Their Application (CNNA-96), Seville, Spain, 24-26 June, pp. 291-296.

Sbitnev, V. I., Yang, T. \& Chua, L. O. [2001] "The local activity criteria for "difference-equation' CNN," Int. J. Bifurcation and Chaos 11(2), 311-419.

Special issue [1992] "Cellular neural network," Int. J. Circuit Th. Appl. 20 (9/10).

Special issue [1993] "Cellular neural network," IEEE Trans. Circuits Systs. I \& II 40(3).

Takahashi, N. \& Chua, L. O. [1997] "A new sufficient 
condition for nonsymmetric CNN's to have a stable equilibrium point," IEEE Trans. Circuits Syst. 44, 1092-1095.

Thiran, R., Crounse, K. R., Chua, L. O. \& Hasler, M. [1995] "Pattern formation properties of autonomous cellural neural network," IEEE Trans. Circuits Syst. I 42(10), 757-774.
Werblin, F., Roska, T. \& Chua, L. O. [1994] "The analogic cellular neural network as a bionic eye," Int. J. Circuit Th. Appl. 23, 541-569.

Werblin, F., Jacobs, A. \& Teeters, J. [1996] "The computational eye," IEEE Spectrum, May, 30-37. 